\title{
Deep brain stimulation and the cerebellum
}

Citation for published version (APA):

Moers-Hornikx, V. M. P. (2013). Deep brain stimulation and the cerebellum. [Doctoral Thesis, Maastricht University]. Datawyse / Universitaire Pers Maastricht. https://doi.org/10.26481/dis.20130301vm

Document status and date:

Published: 01/01/2013

DOI:

10.26481/dis.20130301vm

Document Version:

Publisher's PDF, also known as Version of record

\section{Please check the document version of this publication:}

- A submitted manuscript is the version of the article upon submission and before peer-review. There can be important differences between the submitted version and the official published version of record.

People interested in the research are advised to contact the author for the final version of the publication, or visit the DOI to the publisher's website.

- The final author version and the galley proof are versions of the publication after peer review.

- The final published version features the final layout of the paper including the volume, issue and page numbers.

Link to publication

\footnotetext{
General rights rights.

- You may freely distribute the URL identifying the publication in the public portal. please follow below link for the End User Agreement:

www.umlib.nl/taverne-license

Take down policy

If you believe that this document breaches copyright please contact us at:

repository@maastrichtuniversity.nl

providing details and we will investigate your claim.
}

Copyright and moral rights for the publications made accessible in the public portal are retained by the authors and/or other copyright owners and it is a condition of accessing publications that users recognise and abide by the legal requirements associated with these

- Users may download and print one copy of any publication from the public portal for the purpose of private study or research.

- You may not further distribute the material or use it for any profit-making activity or commercial gain

If the publication is distributed under the terms of Article $25 \mathrm{fa}$ of the Dutch Copyright Act, indicated by the "Taverne" license above, 


\title{
Deep brain stimulation
}

\author{
and
}

the cerebellum 
(C) Véronique M.P. Moers-Hornikx, Maastricht 2013

Layout: Tiny Wouters

Production: Datawyse/Universitaire Pers Maastricht

ISBN: 978-90-9026934-4

The printing of this thesis was financially supported by Eisai BV. 


\title{
Deep brain stimulation
} and

\author{
the cerebellum
}

PROEFSCHRIFT

Ter verkrijging van de graad van doctor aan de Universiteit Maastricht, op gezag van de Rector Magnificus,

Prof. dr. L.L.G. Soete,

volgens het besluit van het College van Decanen,

in het openbaar te verdedigen

op vrijdag 1 maart 2013 om 14:00 uur

door

Véronique Maria Peter Moers-Hornikx 
Promotores

Prof. dr. J.S.H. Vles

Prof. dr. Y. Temel

Co-promotor

Dr. G. Hoogland

Beoordelingscommissie

Prof. dr. R.J. van Oostenbrugge (voorzitter)

Prof. dr. A. Benazzouz (Bordeaux, Frankrijk)

Dr. C. Catsman-Berrevoets (Erasmus medisch centrum Rotterdam)

Prof. dr. J.J. van Overbeeke

Prof. dr. R.J. Stokroos 


\section{Contents}

Chapter $1 \quad$ Introduction 7

Chapter 2 Cerebellar nuclei are involved in impulsive behaviour

Chapter 3 Cerebellar nuclei are activated by high-frequency

stimulation of the subthalamic nucleus

Chapter $4 \quad$ Periaqueductal grey stimulation induced panic-like

behaviour is accompanied by deactivation of the deep

cerebellar nuclei

Chapter 5 c-Fos expression in the deep cerebellar nuclei in

a rat model of conditioned fear

Chapter $6 \quad$ Sustained reduction of cerebellar activity in

experimental epilepsy

Chapter 7 General discussion and conclusion

Summary

Samenvatting

List of Publications

Dankwoord 



\section{Chapter 1}

Introduction 
\begin{tabular}{l|l}
8 & Chapter 1
\end{tabular} 


\section{The cerebellum}

\section{Anatomy}

The cerebellum is a remarkable organ that is located in the posterior fossa. It has a uniform cellular structure across the whole cortex and contains about 50 billion neurons, approximately half of all neurons in the brain. This suggests that the cerebellum is capable of powerful information processing ${ }^{1}$. Comparative anatomical studies have shown that the gross anatomy of the cerebellum in mammals is more or less similar ${ }^{2}$. The cerebellar surface shows numerous horizontal folds called folia. These are interrupted by fissures. The deep primary fissure divides the cerebellum in an anterior lobe and a posterior lobe, while the posterolateral fissure separates the flocculonodular lobe from the posterior lobe (Figure 1.1a). In the posterior lobe there is a clear division in a central vermis and two lateral hemispheres. In the anterior lobe no clear vermis can be discerned, although slight dentations can be seen caused by the paravermian vein ${ }^{2}$. Shallower fissures lead to a division of the vermis and corresponding parts of the hemispheres into ten lobules which have received names from several anatomists but are now often denoted by roman numerals as proposed by Larsell $^{3}$ (Figure 1.1b).

The cerebellar cortex has a specific cellular organisation which is uniform across the whole cerebellum (Figure 1.1c). It is divided into three layers, containing five cell types. Most conspicuous are the Purkinje cells, large cells arranged side by side in a single layer between the molecular layer and the granule cell layer. Their dendrites are highly branched and located in the molecular layer in one plane perpendicular to the direction of the folia. Each Purkinje cell has one axon which traverses the white matter to the deep cerebellar nuclei, where they exert an inhibitory influence on the nuclear cells. The Purkinje cells receive two main inputs. Indirect output comes from the pontine nuclei in the form of mossy fibres that synapse with the granule cells in the granule cell layer. These small glutamatergic cells make up about $95 \%$ of all cerebellar neurons. They send their axons into the molecular layer where they branch and travel in two directions parallel to the direction of the folia. These axons, which are called parallel fibres, form synapses with the dendritic trees of the Purkinje cells, exerting an excitatory influence. The other input to the Purkinje cells derives directly from the inferior olive. These climbing fibres each form multiple excitatory synapses with the dendritic tree of a single Purkinje cell. The only neurons located in the molecular layer are the stellate cells, a subtype of which is the basket cell. These inhibitory interneurons receive input from the granule cells and pass this on to the Purkinje cells. Another type of interneuron is the Golgi cell, which has its cell body in the granule cell layer and, like the Purkinje cells, has a branched dendrite tree in the molecular layer. Their dendrites are contacted mostly by parallel fibres. The axons of the Golgi cells remain in the cerebellar cortex and contact the dendrites of granule cells. 


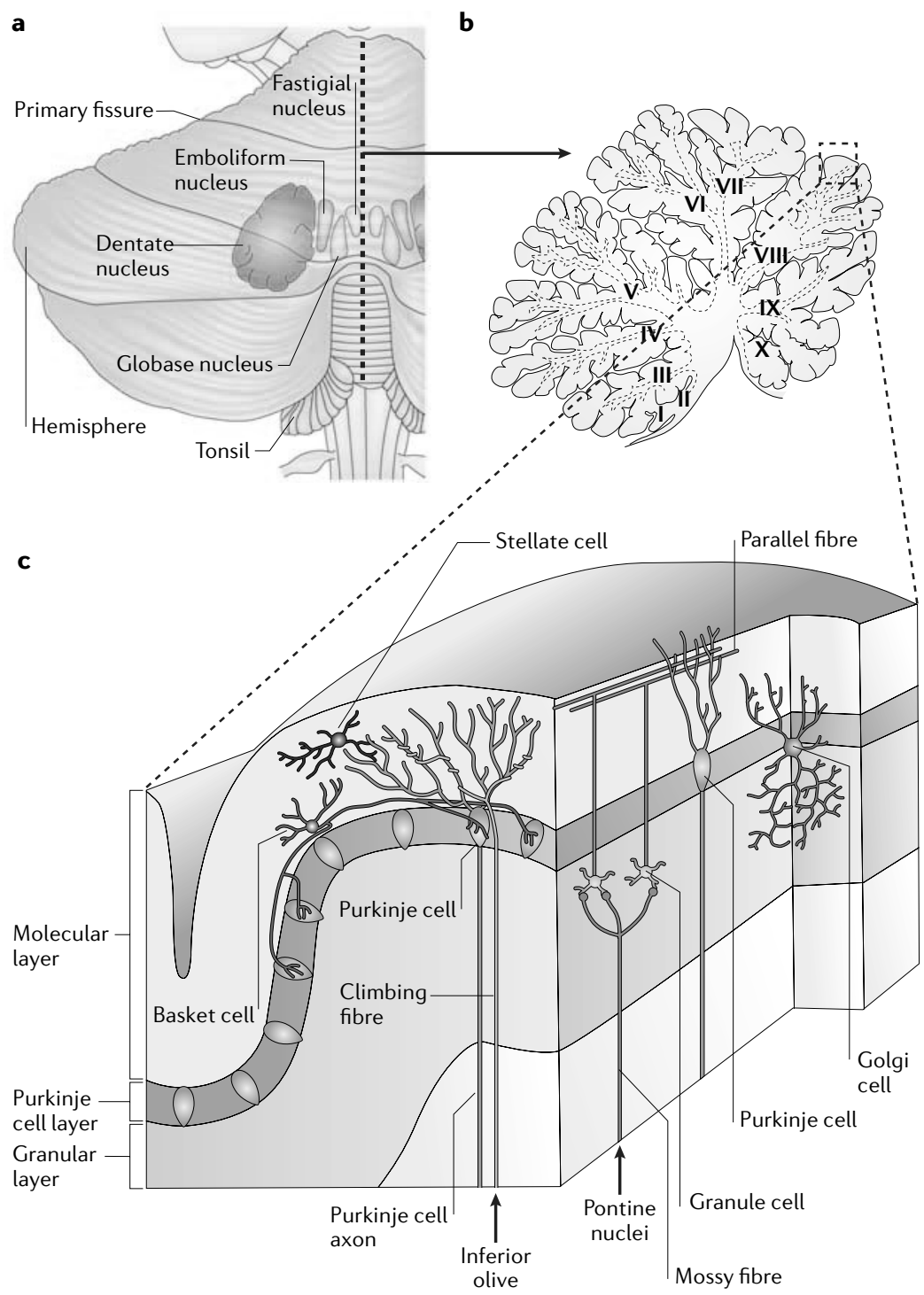

Figure 1.1 Anatomical architecture of the cerebellum. a: Posterior view of the human cerebellum, showing the cerebellar nuclei embedded below the cerebellar cortex. b: Drawing of midsagittal cross-section through the human cerebellum (dotted line indicates the plane of section), showing lobular organization. Each of the ten lobules is demarcated by a Roman numeral $(I-X)$. c: The microstructural organization of the cerebellar cortex. Cut-away illustration of an individual cerebellar cortical lobule, indicating the presence of three layers. The figure shows the relative positions of Purkinje cells and their main inputs (parallel and climbing fibres). Reprinted with permission from Ramnani N. Nat Rev Neurosci. 2006. 
Deep in the white matter several clusters of neurons form the cerebellar nuclei (Figure 1.1a). These structures form the output channels of the cerebellum. They receive input mainly from the Purkinje cells in the cerebellar cortex; however, both mossy fibres and climbing fibres send collaterals directly to the deep cerebellar nuclei. In humans there are four cerebellar nuclei: most medial is the fastigial nucleus, followed by the small globose nucleus and emboliform nucleus. Most lateral is the dentate nucleus. In most mammals four nuclei can also be identified: a medial (or fastigial) nucleus, a posterior interpositus nucleus, an anterior interpositus nucleus and a lateral (or dentate) nucleus.

\section{Function}

The function of the cerebellum has been extensively investigated in the past centuries. In the beginning of the nineteenth century Flourens was the first to propose that the cerebellum is involved in motor coordination ${ }^{4,5}$. This was confirmed by succeeding research. Gradually it has become clear that the cerebellum plays a role in many other processes. Not only motor coordination but also motor learning has been located to the cerebellum ${ }^{6}$. It has been shown to influence autonomic function such as heart rate, blood pressure and micturition ${ }^{7}$. Independent of the influence on autonomic function is a role in fear and fear conditioning ${ }^{8,9}$. Clinical observations and studies have implicated the cerebellum in several psychiatric disorders ${ }^{10}$, and lesions of the cerebellum have been shown to cause a variety of cognitive and behavioural symptoms. The latter has been called the cerebellar cognitive affective syndrome $(\mathrm{CCAS})^{11}$. A role for the cerebellum in epilepsy was suspected in the beginning of the twentieth century when Hunt described coexistence of myoclonic activity and cerebellar atrophy in patients ${ }^{12}$. Many studies have analysed the effect of cerebellar lesions and stimulation on seizure generation and spread, with inconsistent results ${ }^{13,14}$.

Traditionally the cerebellum was thought to integrate input from multiple cortical areas leading to a single output to the primary motor cortex ${ }^{15}$. Parallel to the elucidation of the complex functions of the cerebellum it has been shown that the cerebellum projects to many more cortical and subcortical areas. In the research on its role in autonomic functions reciprocal connections between the cerebellum and the hypothalamus have been shown ${ }^{16}$. Projections from the cerebellum to the periaqueductal grey have been shown in the rat, connecting the cerebellum with the fear network ${ }^{17}$. It has become increasingly clear that the connections between the cerebellum and the cortex are also reciprocal, forming separate closed loop circuits which likely subserve different functions ${ }^{18}$. Besides connections with the primary motor cortex, such closed loop circuits have also been shown between the cerebellum and the premotor, prefrontal and parietal cortical areas, supporting a role of the cerebellum in cognitive functions $^{18}$. Projections from the cerebellum to the amygdala have been suspected based on behavioural changes due to cerebellar lesions, but an anatomical pathway has never been shown ${ }^{19}$. Recent tracing experiments have shown 
that there is a closed loop between the dentate nucleus of the cerebellum and the basal ganglia which is independent of cortical input ${ }^{20}$.

The deep cerebellar nuclei are the output channels of the cerebellum. Each nucleus is thought to have its specific connections and function. Lesion studies have shown that only the fastigial nucleus is involved in heart rate conditioning ${ }^{8,21,22}$. Lesions of the dentate and interpositus nucleus interfere with learning of conditioned responses but have no effect on heart rate conditioning ${ }^{8}$. In rats, projections to the hypothalamus arise from all three nuclei ${ }^{16}$, although the majority arise from the dentate nucleus ${ }^{16,17}$. Historically the dentate nucleus was considered to be the only cerebellar nucleus to project to the cerebral cortex ${ }^{15,23}$. However, it was shown that in non-human primates all three nuclei project to cerebral cortex through the ventrolateral thalamic nucleus $(\mathrm{VL})$, the centrolateral thalamic nucleus $(\mathrm{CL})$ and the mediodorsal thalamic nucleus $(\mathrm{MD})^{23,24}$. The cerebellar-recipient areas of these thalamic nuclei have been shown to project to the primary motor cortex in a somatotopic fashion ${ }^{25}$. Projections to the prefrontal cortex originate in the dentate nucleus ${ }^{26,27}$. The projections from the dentate nucleus to the premotor cortex derive from a different part of this nucleus than the projections to the primary motor $\operatorname{cortex}^{28,29}$, suggesting the existence of motor and non-motor domains in the dentate nucleus. This was already suggested by Leiner et al. based on the existence of two anatomically different subdivisions in the dentate nucleus ${ }^{24}$ and is supported by the finding that the relative increase in size of the ventral part of the dentate nucleus when comparing humans to great apes is disproportionally large compared to the dorsal part of the dentate nucleus ${ }^{30}$. In relation to epilepsy both the fastigial nucleus and the dentate nucleus have been implicated ${ }^{31}$.

\section{Cerebellar activity during deep brain stimulation in Parkinson's disease}

Deep brain stimulation (DBS) has become an accepted treatment option for several movement disorders and is being investigated as a potential treatment option in psychiatric disorders ${ }^{32}$. The experience with DBS is largest in patients with Parkinson's disease (PD). The subthalamic nucleus (STN), the internal segment of the globus pallidus (GPi) and the ventrointermediate nucleus of the thalamus (VIM) have all been targeted with good effects on tremor, rigidity and bradykinesia. Recently the pedunculopontine nucleus (PPN) has also been targeted with promising effects on axial symptoms $^{33}$. The mechanisms underlying the therapeutic effects of DBS are under investigation. In patients functional imaging using PET and SPECT provides a method to investigate the effects of DBS in vivo. In these studies changes of activation are frequently seen in the cerebellum, as summarized below. 


\section{Subthalamic nucleus}

In studies reporting changes in cerebellar blood flow or metabolism during STN DBS at rest, results are inconsistent. Several groups report decreased regional cerebral blood flow (rCBF) or glucose metabolism ${ }^{34-38}$, while others show increases ${ }^{39-47}$. Changes are reported in the cerebellar hemispheres as well as in the vermis. One group showed increased glucose metabolism in the anterior cerebellar cortex and decreases in the posterior cortex ${ }^{48,49}$. Functional imaging during STN DBS while performing a movement task shows increased activation in the cerebellum ipsilateral to movement and decreases contralateral to movement ${ }^{50,51}$.

\section{Pallidal complex}

One group reported changes in activity in the cerebellum during DBS of the internal segment of the globus pallidus (GPi) ${ }^{52}$. They describe an increase of rCBF bilaterally in the cerebellum using FDG-PET in rest. In the same patients improvement of several aspects of a motor task correlated with increased activity in both cerebellar hemispheres on $\mathrm{H}_{2}{ }^{15} \mathrm{O}-\mathrm{PET}^{53}$. In another study examining the effect of unilateral GPi and GPe DBS, no changes were seen in the cerebellum during GPi stimulation ${ }^{54}$. However, GPe stimulation led to decreased blood flow in the cerebellum ipsilateral to the side of stimulation.

\section{Ventrointermediate nucleus of the thalamus}

Two studies performing $\mathrm{H}_{2}{ }^{15} \mathrm{O}-\mathrm{PET}$ during unilateral DBS of the ventrointermediate nucleus of the thalamus (VIM) show decreased activity in the contralateral cerebellar hemisphere ${ }^{55,56}$. Deiber et al. showed decreased $\mathrm{rCBF}$ in the anterior vermis and anterior part of the intermediate zone of the cerebellar hemispheres ${ }^{57}$.

\section{Pedunculopontine nucleus}

The pedunculopontine nucleus (PPN) has recently been added as a possible target for DBS in PD, with effects especially on axial symptoms. In a case report, Strafella et al. show increased $\mathrm{rCBF}$ in the ipsilateral cerebellum during unilateral PPN DBS ${ }^{58}$. In a subsequent study of three patients the same group showed rCBF increase bilaterally in the cerebellum ${ }^{59}$. In a case report of a patient with freezing of gait PPN DBS led to an increase of connectivity with the cerebellum as measured by diffusion tensor imaging (DTI), restoring a pattern that resembles that of healthy controls ${ }^{60}$. 


\section{Cerebellar changes following deep brain stimulation: animal research}

Functional imaging in patients is an indirect method to examine the effects of DBS on the brain. Animal models provide a method to further examine local and distant effects of DBS permitting histological as well as functional analysis. Animal models using DBS have been used to analyse functional connectivity of the stimulated structures as well as to elucidate the mechanism of DBS. Furthermore, stimulation of several forebrain structures leads to the development of epileptic seizures. This process called kindling is used as an animal model to study the pathophysiology of epilepsy and the effect of treatment.

\section{Literature search}

We searched the available literature for animal studies analysing changes in the cerebellum following deep brain stimulation including kindling. We used the following search terms and various combinations of these terms: deep brain stimulation, DBS, high frequency stimulation, HFS, cerebellum, cerebellar nuclei, dentate nucleus, interpositus nucleus, fastigial nucleus, epilepsy, amygdala, kindling, subthalamic nucleus, STN, thalamus, ventrolateral nucleus, mediodorsal nucleus, periaqueductal grey, PAG. Results were limited to animal research and only papers written in English were included. References of relevant papers were screened to find additional papers. We found 14 papers which fit our objective. The results described in these papers are summarized below.

\section{Substantia nigra}

In a study using a quantitative autoradiographic 2-deoxy-D-[1- $\left.{ }^{14} \mathrm{C}\right]$ glucose method $\left({ }^{14} \mathrm{C}-\mathrm{DG}\right)$ Savaki et al. analysed the local glucose utilisation in rats following unilateral stimulation of the substantia nigra $(S N)^{61}$. They found a significant change in both ipsilateral and contralateral dentate nucleus (DN) and interpositus nucleus (IN) of the cerebellum. Additional changes in the vermis were attributed to motor behavioural manifestations seen in the conscious stimulated animals since these changes in metabolic activity following stimulation were not found in halothane anesthetized animals. Changes in metabolic activity in the DN and IN following SN stimulation were thought to be the result of orthodromic activation of direct efferents of the SN to the deep cerebellar nuclei (DCbN).

\section{Subthalamic nucleus}

Using the same autoradiographic method mentioned above, Tzagournissakis et al. ${ }^{62}$ found no significant alterations of glucose metabolism in the molecular or granular layer of the cerebellum following unilateral DBS of the STN in rats. 


\section{Thalamus}

Raos et al. analysed local cerebral glucose utilization following unilateral stimulation of the intralaminar centrolateral thalamic nucleus $(\mathrm{CL})$ in rats ${ }^{63}$. They found a tendency to increased metabolism in the contralateral fastigial and interpositus cerebellar nuclei. These results showed significant correlation with the extent of locally induced CL-stimulation effect. Gao et al. showed that neither unilateral nor bilateral DBS of the anterior thalamic nucleus (ATN) leads to changes in glucose uptake levels in the cerebellum in rats ${ }^{64}$.

\section{Amygdala}

Kindling is a model of epilepsy in which repeated subthreshold electrical stimulation of a certain brain region leads to a build-up of seizure activity, culminating in full fledged epileptic seizures ${ }^{65}$. A frequently used target is the amygdala. Amygdala kindling is used to study the pathophysiology of temporal lobe epilepsy and the effects of treatment with novel antiepileptic drugs as well as vagus nerve stimulation ${ }^{65,66}$. Animal studies have shown that cerebellar lesions and cerebellar stimulation interfere with the kindling process ${ }^{31,67}$. However, few groups have looked at the effects of amygdala kindling on the cerebellum. Of those studies that included the cerebellum in their analysis, most do not offer a rationale for this analysis or consider the cerebellum to be a control region. Studies analysing the glucose utilization ${ }^{68}$, calcium binding protein (CaBP) levels and gene expression ${ }^{69}$, neuronal degeneration and changes in astrocytes and microglia ${ }^{70}$ found no changes in the cerebellum in partially and fully kindled rats. Several studies have analyzed the role of neurotransmitters in the kindling model. No changes in GABA levels or GAD gene expression and activity were found in the cerebellum of fully kindled rats ${ }^{69,71,72}$. In contrast, Tietz and Chiu found a change in GABAergic function in the cerebellum ${ }^{73}$. Synaptosomal uptake of taurine increased in the cerebellum of fully kindled rats ${ }^{71}$. Teskey et al. reported slightly enhanced fos expression in the cerebellum one hour after a stage 5 seizure in fully kindled rats ${ }^{74}$. Seegers et al. examined the expression of multidrug transporter P-glycoprotein (PGP) using the cerebellum as a control region; their only significant finding was a larger PGP-labelled area in the contralateral cerebellum of kindled rats ${ }^{75}$.

\section{Entorhinal cortex}

One group performed entorhinal cortex kindling and analysed cytochrome oxidase activity 24 and 48 hours after the last seizure in fully kindled rats. They found no changes in any brain region including the cerebellum ${ }^{76}$. 


\section{Aims of the thesis}

In this study, we investigated the role of the deep cerebellar nuclei in motor, cognitive and emotional behaviour. We performed a set of experiments in which we modulated motor, cognitive and emotional behaviour using DBS, and investigated the neuronal activities in the deep cerebellar nuclei, utilising animal models. Our main aim was to advance our understanding on the functional roles of the deep cerebellar nuclei in different forms of behaviour linked to different brain regions.

In chapter $\mathbf{2}$ we report on the effect of DBS of the mediodorsal thalamic nucleus $(\mathrm{MD})$ and the ventrolateral thalamic nucleus $(\mathrm{VL})$ in rats on impulsive behaviour and on neuronal activity in the deep cerebellar nuclei (DCbN) and prefrontal cortex (PFC). The hypothesis that inhibition of the DCbN leads to abnormal behaviour is explored further in chapters 3 through $\mathbf{6}$. In chapter $\mathbf{3}$ we describe the effect of STN DBS, which is known to enhance motor behaviour and to decrease impulsivity in rats ${ }^{77}$, on neuronal activity in the DCbN. DBS of the dorsolateral periaqueductal grey (dIPAG) and the ventromedial hypothalamus (VMH) in rats has been shown to mimic panic attacks in humans $^{78,79}$. The effect of this stimulation model on neuronal activity in the DCbN is described in chapter 4 . In chapter 5 we report the effect of conditioned panic attacks on neuronal activity in the $\mathrm{DCbN}$. In chapter 6 we describe the effect of amygdala kindling, a model of chronic epilepsy, on neuronal activity and metabolic changes in the $\mathrm{DCbN}$, and correlate these results to kindling parameters. Finally, in chapter $\mathbf{7}$ we present a summary of the findings in this thesis and the implications of these findings are discussed. 


\section{References}

1. Ramnani N. The primate cortico-cerebellar system: anatomy and function. Nat Rev Neurosci. 2006;7:511-22.

2. Voogd J, Glickstein M. The anatomy of the cerebellum. Trends Neurosci. 1998;21:370-5.

3. Larsell O, Jansen J, Korneliussen HK, Mugnaini E. The Comparative Anatomy and Histology of the Cerebellum: The Human Cerebellum, Cerebellar Connections, and Cerebellar Cortex: University of Minnesota Press; 1972.

4. Fine EJ, Ionita CC, Lohr L. The history of the development of the cerebellar examination. Semin Neurol. 2002;22:375-84.

5. Glickstein M, Strata P, Voogd J. Cerebellum: history. Neuroscience. 2009;162:549-59.

6. Ito M. Historical review of the significance of the cerebellum and the role of Purkinje cells in motor learning. Ann N Y Acad Sci. 2002;978:273-88.

7. Dietrichs E, Haines DE. Possible pathways for cerebellar modulation of autonomic responses: micturition. Scand J Urol Nephrol Suppl. 2002;(210):16-20.

8. Lavond DG, Lincoln JS, McCormick DA, Thompson RF. Effect of bilateral lesions of the dentate and interpositus cerebellar nuclei on conditioning of heart-rate and nictitating membrane/eyelid responses in the rabbit. Brain Res. 1984;305:323-30.

9. Sacchetti B, Scelfo B, Strata P. The cerebellum: synaptic changes and fear conditioning. Neuroscientist. 2005;11:217-27.

10. Hoppenbrouwers SS, Schutter DJ, Fitzgerald PB, Chen R, Daskalakis ZJ. The role of the cerebellum in the pathophysiology and treatment of neuropsychiatric disorders: a review. Brain Res Rev. 2008;59: 185-200.

11. Schmahmann JD, Sherman JC. The cerebellar cognitive affective syndrome. Brain. 1998;121: 561-79.

12. Dow RS, Fernandez-Guardiola A, Manni E. The influence of the cerebellum on experimental epilepsy. Electroencephalogr Clin Neurophysiol. 1962;14:383-98.

13. Fountas KN, Kapsalaki E, Hadjigeorgiou G. Cerebellar stimulation in the management of medically intractable epilepsy: a systematic and critical review. Neurosurg Focus. 2010;29:E8.

14. Saillet S, Langlois M, Feddersen B, Minotti L, Vercueil L, Chabardès S, David O, Depaulis A, Deransart C, Kahane $P$. Manipulating the epileptic brain using stimulation: a review of experimental and clinical studies. Epileptic Disord. 2009;11:100-12.

15. Allen GI, Tsukahara N. Cerebrocerebellar communication systems. Physiol Rev. 1974;54:957-1006.

16. Çavdar S, San T, Aker R, Sehirli ÜS, Onat FYI. Cerebellar connections to the dorsomedial and posterior nuclei of the hypothalamus in the rat. J Anat. 2001;198:37-45.

17. Teune TM, van der Burg J, van der Moer J, Voogd J, Ruigrok TJ. Topography of cerebellar nuclear projections to the brain stem in the rat. Prog Brain Res. 2000;124:141-72.

18. Kelly RM, Strick PL. Cerebellar loops with motor cortex and prefrontal cortex of a nonhuman primate. J Neurosci. 2003;23:8432-44.

19. Strick PL, Dum RP, Fiez JA. Cerebellum and nonmotor function. Annu Rev Neurosci. 2009;32:413-34.

20. Bostan AC, Strick PL. The cerebellum and basal ganglia are interconnected. Neuropsychol Rev. 2010;20:261-70.

21. Supple WF, Jr., Leaton RN. Lesions of the cerebellar vermis and cerebellar hemispheres: effects on heart rate conditioning in rats. Behav Neurosci. 1990;104:934-47.

22. Timmann D, Drepper J, Frings M, Maschke M, Richter S, Gerwig M, Kolb FP. The human cerebellum contributes to motor, emotional and cognitive associative learning. A review. Cortex. 2010;46:845-57.

23. Asanuma C, Thach WT, Jones EG. Distribution of cerebellar terminations and their relation to other afferent terminations in the ventral lateral thalamic region of the monkey. Brain Res. 1983;286:237-65.

24. Leiner HC, Leiner AL, Dow RS. Does the cerebellum contribute to mental skills? Behav Neurosci. 1986;100:443-54.

25. Hoover JE, Strick PL. The organization of cerebellar and basal ganglia outputs to primary motor cortex as revealed by retrograde transneuronal transport of herpes simplex virus type 1 . J Neurosci. 1999;19:1446-63.

26. Middleton FA, Strick PL. Anatomical evidence for cerebellar and basal ganglia involvement in higher cognitive function. Science. 1994;266:458-61. 
27. Middleton FA, Strick PL. Basal ganglia and cerebellar loops: motor and cognitive circuits. Brain Res Rev. 2000;31:236-50.

28. Dum RP, Li C, Strick PL. Motor and nonmotor domains in the monkey dentate. Ann N Y Acad Sci. 2002;978:289-301.

29. Dum RP, Strick PL. An unfolded map of the cerebellar dentate nucleus and its projections to the cerebral cortex. J Neurophysiol. 2003;89:634-9.

30. Matano S. Brief communication: Proportions of the ventral half of the cerebellar dentate nucleus in humans and great apes. Am J Phys Anthropol. 2001;114:163-5.

31. Min JK, Valentine PA, Teskey GC. Effect of complete and partial bilateral lesions of the deep cerebellar nuclei on amygdaloid kindling in rats. Epilepsia. 1998;39:692-9.

32. Benabid AL, Wallace B, Mitrofanis J, Xia C, Piallat B, Fraix V, Batir A, Krack P, Pollak P, Berger F. Therapeutic electrical stimulation of the central nervous system. C R Biol. 2005;328:177-86.

33. Fasano A, Daniele A, Albanese A. Treatment of motor and non-motor features of Parkinson's disease with deep brain stimulation. Lancet Neurol. 2012;11:429-42.

34. Asanuma K, Tang C, Ma Y, Dhawan V, Mattis P, Edwards C, Kaplitt MG, Feigin A, Eidelberg D. Network modulation in the treatment of Parkinson's disease. Brain. 2006;129:2667-78.

35. Geday J, Ostergaard K, Johnsen E, Gjedde A. STN-stimulation in Parkinson's disease restores striatal inhibition of thalamocortical projection. Human Brain Mapping. 2009;30: 112-21.

36. Hershey T, Revilla FJ, Wernle AR, McGee-Minnich L, Antenor JV, Videen TO, Dowling JL, Mink JW, Perlmutter JS. Cortical and subcortical blood flow effects of subthalamic nucleus stimulation in PD. Neurology. 2003;61:816-21.

37. Trost M, Su S, Su P, et al. Network modulation by the subthalamic nucleus in the treatment of Parkinson's disease. Neuroimage. 2006;31:301-7.

38. Wang J, Ma Y, Huang Z, Sun B, Guan Y, Zuo C. Modulation of metabolic brain function by bilateral subthalamic nucleus stimulation in the treatment of Parkinson's disease. J Neurol. 2010;257:72-8.

39. Cilia R, Marotta G, Landi A, Isaias IU, Mariani CB, Vergani F, Benti R, Sganzerla E, Pezzoli G, Antonini A. Clinical and cerebral activity changes induced by subthalamic nucleus stimulation in advanced Parkinson's disease: a prospective case-control study. Clin Neurol Neurosurg. 2009;111:140-6.

40. Le Jeune F, Drapier D, Bourguignon A, Péron J, Mesbah H, Drapier S, Sauleau P, Haegelen C, Travers D, Garin E, Malbert CH, Millet B, Vérin M. Subthalamic nucleus stimulation in Parkinson disease induces apathy: a PET study. Neurology. 2009;73:1746-51.

41. Le Jeune F, Péron J, Biseul I, Fournier S, Sauleau P, Drapier S, Haegelen C, Drapier D, Millet B, Garin E, Herry JY, Malbert $\mathrm{CH}$, Vérin M. Subthalamic nucleus stimulation affects orbitofrontal cortex in facial emotion recognition: a PET study. Brain. 2008;131:1599-608.

42. Le Jeune F, Péron J, Grandjean D, Drapier S, Haegelen C, Garin E, Millet B, Vérin M. Subthalamic nucleus stimulation affects limbic and associative circuits: a PET study. Eur J Nucl Med Mol Imaging. 2010;37:1512-20.

43. Phillips MD, Baker KB, Lowe MJ, Tkach JA, Cooper SE, Kopell BH, Rezai AR. Parkinson disease: pattern of functional MR imaging activation during deep brain stimulation of subthalamic nucleus - initial experience. Radiology. 2006;239:209-16.

44. Sestini S, Ramat S, Formiconi AR, Ammanati F, Sorbi S, Pupi A. Brain networks underlying the clinical effects of long-term subthalamic stimulation for Parkinon's disease: A 4-year follow-up study with rCBF SPECT. J Nucl Med. 2005;46:1444-54.

45. Stefurak T, Mikulis D, Mayberg H, Lang AE, Hevenor S, Pahapill P, Saint-Cyr J, Lozano A. Deep brain stimulation for Parkinson's disease dissociates mood and motor circuits: a functional MRI case study. Mov Disord. 2003;18: 1508-16.

46. Tanei T, Kajita Y, Nihashi T, Kaneoke Y, Takebayashi S, Nakatsubo D, Wakabayashi T. Changes in regional blood flow induced by unilateral subthalamic nucleus stimulation in patients with Parkinson's disease. Neurol Med Chir (Tokyo). 2009;49:507-13.

47. Vafaee MS, K OS, Sunde N, Gjedde A, Dupont E, Cumming P. Focal changes of oxygen consumption in cerebral cortex of patients with Parkinson's disease during subthalamic stimulation. Neuroimage. 2004;22:966-74. 
48. Hilker R, Voges J, Weisenbach S, Kalbe E, Burghaus L, Ghaemi M, Lehrke R, Koulousakis A, Herholz K, Sturm V, Heiss WD. Subthalamic nucleus stimulation restores glucose metabolism in associative and limbic cortices and in cerebellum: evidence from a FDG-PET study in advanced Parkinson's disease. J Cereb Blood Flow Metab. 2004;24:7-16.

49. Hilker R, Voges J, Thiel A, Ghaemi M, Herholz K, Sturm V, Heiss WD. Deep brain stimulation of the subthalamic nucleus versus levodopa challenge in Parkinson's disease: measuring the on- and offconditions with FDG-PET. J Neural Transm. 2002;109:1257-64.

50. Grafton ST, Turner RS, Desmurget M, Bakay R, Delong M, Vitek J, Crutcher M. Normalizing motorrelated brain activity; Subthalamic nucleus stimulation in Parkinson disease. Neurology. 2006;66: 1192-9.

51. Payoux P, Remy P, Damier P, Miloudi M, Loubinoux I, Pidoux B, Gaura V, Rascol O, Samson Y, Agid Y. Subthalamic Nucleus Stimulation Reduces Abnormal Motor Cortical Activity in Parkinson Disease. Arch Neurol. 2004;61:1307-13.

52. Fukuda M, Mentis MJ, Ma Y, et al. Networks mediating the clinical effects of pallidal brain stimulation for Parkinson's disease: a PET study of resting-state glucose metabolism. Brain. 2001;124:1601-9.

53. Fukuda M, Mentis MJ, Ma Y, Dhawan V, Antonini A, Lang AE, Lozano AM, Hammerstad J, Lyons K, Koller WC, Moeller JR, Eidelberg D. Functional correlates of pallidal stimulation for Parkinson's disease. Ann Neurol. 2001;49:155-64.

54. Payoux P, Remy P, Miloudi M, Houeto JL, Stadler C, Bejjani BP, Yelnik J, Samson Y, Rascol O, Agid Y, Damier $\mathrm{P}$. Contrasting changes in cortical activation induced by acute high-frequency stimulation within the globus pallidus in Parkinson's disease. J Cereb Blood Flow Metab. 2009;29:235-43.

55. Davis KD, Taub E, Houle S, Lang AE, Dostrovsky JO, Tasker RR, Lozano AM. Globus pallidus stimulation activates the cortical motor system during alleviation of parkinsonian symptoms. Nat Med. 1997;3:671-4.

56. Fukuda M, Barnes A, Simon ES, Holmes A, Dhawan V, Giladi N, Fodstad H, Ma Y, Eidelberg D. Thalamic stimulation for parkinsonian tremor: correlation between regional cerebral blood flow and physiological tremor characteristics. Neuroimage. 2004;21:608-15.

57. Deiber MP, Pollak P, Passingham R, Landais P, Gervason C, Cinotti L, Friston K, Frackowiak R, Mauguière $F$, Benabid AL. Thalamic stimulation and suppression of parkinsonian tremor. Evidence of $a$ cerebellar deactivation using positron emission tomography. Brain. 1993;116:267-79.

58. Strafella AP, Lozano AM, Ballanger B, Poon YY, Lang AE, Moro E. rCBF changes associated with PPN stimulation in a patient with Parkinson's disease: a PET study. Mov Disord. 2008;23:1051-4.

59. Ballanger B, Lozano AM, Moro E, van Eimeren T, Hamani C, Chen R, Cilia R, Houle S, Poon YY, Lang AE, Strafella AP. Cerebral blood flow changes induced by pedunculopontine nucleus stimulation in patients with advanced Parkinson's disease: a [(15)O] H2O PET study. Hum Brain Mapp. 2009;30:3901-9.

60. Schweder PM, Joint C, Hansen PC, Green AL, Quaghebeur G, Aziz TZ. Chronic pedunculopontine nucleus stimulation restores functional connectivity. Neuroreport. 2010;21: 1065-8.

61. Savaki HE, Desban M, Glowinski J, Besson MJ. Local cerebral glucose consumption in the rat. II. Effects of unilateral substantia nigra stimulation in conscious and in halothane-anesthetized animals. J Comp Neurol. 1983;213:46-65.

62. Tzagournissakis M, Dermon CR, Savaki HE. Functional metabolic mapping of the rat brain during unilateral electrical stimulation of the subthalamic nucleus. J Cereb Blood Flow Metab. 1994;14: 132-44.

63. Raos VC, Dermon CR, Savaki HE. Functional anatomy of the thalamic centrolateral nucleus as revealed with the $[14 \mathrm{C}]$ deoxyglucose method following electrical stimulation and electrolytic lesion. Neuroscience. 1995;68:299-313.

64. Gao F, Guo Y, Zhang H, Wang S, Wang J, Wu JM, Chen Z, Ding MP. Anterior thalamic nucleus stimulation modulates regional cerebral metabolism: an FDG-MicroPET study in rats. Neurobiol Dis. 2009;34:477-83.

65. Racine RJ. Modification of seizure activity by electrical stimulation. II. Motor seizure. Electroencephalogr Clin Neurophysiol. 1972;32:281-94.

66. Rijkers K, Aalbers M, Hoogland G, van Winden L, Vles J, Steinbusch H, Majoie M. Acute seizuresuppressing effect of vagus nerve stimulation in the amygdala kindled rat. Brain Res. 2010;1319: 155-63. 
67. Wang S, Wu DC, Ding MP, Li Q, Zhuge ZB, Zhang SH, Chen Z. Low-frequency stimulation of cerebellar fastigial nucleus inhibits amygdaloid kindling acquisition in Sprague-Dawley rats. Neurobiol Dis. 2008;29:52-8.

68. Blackwood DH, Kapoor V, Martin MJ. Regional changes in cerebral glucose utilization associated with amygdaloid kindling and electroshock in the rat. Brain Res. 1981;224:204-8.

69. Sonnenberg JL, Frantz GD, Lee S, Heick A, Chu C, Tobin AJ, Christakos S. Calcium binding protein (calbindin-D28k) and glutamate decarboxylase gene expression after kindling induced seizures. Brain Res Mol Brain Res. 1991;9:179-90.

70. Khurgel M, Switzer RC, 3rd, Teskey GC, Spiller AE, Racine RJ, Ivy GO. Activation of astrocytes during epileptogenesis in the absence of neuronal degeneration. Neurobiol Dis. 1995;2:23-35.

71. Fabisiak JP, Schwark WS. Cerebral free amino acids in the amygdaloid kindling model of epilepsy. Neuropharmacology. 1982;21:179-82.

72. Loscher W, Schwark WS. Further evidence for abnormal GABAergic circuits in amygdala-kindled rats. Brain Res. 1987;420:385-90.

73. Tietz EI, Chiu TH. Regional GABA-stimulated chloride uptake in amygdala kindled rats. Neurosci Lett. 1991;123:269-72.

74. Teskey GC, Atkinson BG, Cain DP. Expression of the proto-oncogene c-fos following electrical kindling in the rat. Brain Res Mol Brain Res. 1991;11:1-10.

75. Seegers U, Potschka H, Loscher W. Expression of the multidrug transporter P-glycoprotein in brain capillary endothelial cells and brain parenchyma of amygdala-kindled rats. Epilepsia. 2002;43:675-84.

76. Nobrega JN, Petrasek JS, Raymond R, Dixon LM, Burnham WM. Brain cytochrome oxidase activity after kindled seizures: a quantitative histochemical mapping study. Brain Res. 1993;622:113-8.

77. Desbonnet L, Temel Y, Visser-Vandewalle V, Blokland A, Hornikx V, Steinbusch HW. Premature responding following bilateral stimulation of the rat subthalamic nucleus is amplitude and frequency dependent. Brain Res. 2004;1008:198-204.

78. Lim LW, Blokland A, Visser-Vandewalle V, Vlamings R, Sesia T, Steinbusch H, Schruers K, Griez E, Temel $Y$. High-frequency stimulation of the dorsolateral periaqueductal gray and ventromedial hypothalamus fails to inhibit panic-like behaviour. Behav Brain Res. 2008;193:197-203.

79. Schenberg LC, Bittencourt AS, Sudre EC, Vargas LC. Modeling panic attacks. Neurosci Biobehav Rev. 2001;25:647-59. 


\section{Chapter}

Cerebellar nuclei are involved in impulsive behaviour

VMP Moers-Hornikx, T Sesia, K Basar, LW Lim, G Hoogland, HWM Steinbusch, AWD Gavilanes, Y Temel, JSH Vles

Behav Brain Res. 2009;203:256-63 


\section{Abstract}

Recent anatomical and clinical evidence has shown that the cerebellum, primarily considered a motor control structure, is also involved in higher cognitive functions and behavioural changes, such as impulsive behaviour. Impulsive behaviour has been shown in several studies to be increased by lesions of the mediodorsal (MD) thalamic nucleus. We performed deep brain stimulation (DBS) of the mediodorsal and ventrolateral (VL) thalamic nuclei in rats, clinically mimicking such a lesion, and tested them for changes in impulsive behaviour in a choice reaction time test. We then analysed the effects of this stimulation on c-Fos expression in both the deep cerebellar nuclei $(D C b N)$ and the prefrontal cortex ( $P F C)$, and correlated these outcomes to the measured changes in impulsive behaviour. DBS of the MD thalamic nucleus increased impulsive behaviour without changing motor parameters. This was accompanied by a decrease in the c-Fos expression in all cerebellar nuclei; with a corresponding increase in c-Fos expression in the PFC. DBS of the VL thalamic nucleus caused no significant change in behaviour or c-Fos expression in either region. The present study demonstrates that impulsive behaviour involves the cerebellar nuclei, possibly through a decreased selective attention caused by a disruption of the cerebellothalamo-cortical pathways through the MD thalamic nucleus. 


\section{Introduction}

Since the first description of the cerebellum as a motor structure by Flourens in the beginning of the nineteenth century, the cerebellum has been regarded to be involved in motor coordination and control ${ }^{1}$. Inputs from several brain regions including the motor, premotor, posterior parietal, cingulate and prefrontal cortex (PFC) were shown to converge on the cerebellar cortex and deep cerebellar nuclei $(D C b N)^{2,3}$. It was thought that the deep cerebellar nuclei project strictly to a specific region in the ventrolateral thalamic nucleus $(\mathrm{VL})$, and from there to the primary motor cortex ${ }^{4,5}$. The last two decades, however, there has been increasing attention for a possible nonmotor role of the cerebellum. Leiner and associates first hypothesized that the disproportional increase in size of the phylogenetically newer parts of the cerebellum in anthropoid apes and humans might imply a role of the cerebellum in mental skills in the same manner as the phylogenetically older parts of the cerebellum contribute to motor skills ${ }^{6}$. Neuroanatomical research has shown that the cerebellum projects to the PFC through the VL and other thalamic nuclei including the mediodorsal thalamic nucleus $(\mathrm{MD})^{7,8}$ and the reticular nucleus of the thalamus (RNT) ${ }^{9}$. These anatomical connections between the cerebellum and the PFC suggest that the cerebellum is involved in non-motor circuits.

Other findings supporting the theory of a non-motor role of the cerebellum come from the clinical setting. This was first pointed out by case reports and case series describing behavioural disturbances in patients with cerebellar lesions due to for example stroke or tumours ${ }^{10-13}$. Nowadays there is an increasing amount of information from human neuroimaging studies to support this view of a non-motor role of the cerebellum ${ }^{14,15}$. In this respect, Schmahmann and colleagues have described a constellation of cognitive deficits in a group of patients with selective cerebellar damage which has been termed the cerebellar cognitive affective syndrome (CCAS) ${ }^{14}$. This syndrome consists of impairment of executive functions, difficulties with spatial cognition, changes of personality including disinhibited or inappropriate behaviour, and language deficits. A similar syndrome has been described in children after resection of cerebellar tumours. This cerebellar mutism syndrome (CMS) is characterized by mutism in combination with other neurological, cognitive and behavioural deficits including either decreased initiation of activity or disinhibition ${ }^{11,16-18}$. It has been suggested that CCAS and CMS are in fact different presentations of the same syndrome ${ }^{11,18}$. Additional evidence that the cerebellum plays a role in higher-order behaviour comes from imaging studies showing significantly smaller cerebellar volumes in patients with neuropsychiatric diseases such as ADHD and schizophrenia ${ }^{19-23}$, and altered metabolism in the cerebellum in patients with obsessive compulsive disorder $(O C D)^{24-27}$. There is also an abnormal cerebellar activation on functional MRI in autistic subjects during motor and cognitive tasks ${ }^{28}$. Pathological findings in these subjects include loss of Purkinje cells (PC's) ${ }^{29}$. Impulsivity is a core feature of these neuropsychiatric diseases ${ }^{30}$. As described above, there have 
been several reports of changes in impulsive behaviour as part of the CCAS or CMS in patients with cerebellar damage ${ }^{11,12,14}$. In autism spectrum disorders the degree of explorative behaviour is correlated to the volume of vermal lobules VI-VII of the cerebellum $^{31}$. Duchenne's muscular dystrophy has been shown to have a heightened association with ADHD, autism spectrum disorders and $O C D^{32}$, which is speculated to be caused by the absence of dystrophin in the cerebellum ${ }^{33}$. Furthermore, King and coworkers showed a change of activity in the cerebellar vermis in a rat model of ADHD after treatment with a 5-HT agonist that is known to relieve impulsivity ${ }^{30}$.

These recent findings suggest a key role of the cerebellum in impulsive behaviour. In this study we have tested this hypothesis. Previous research has shown that lesions of the MD thalamic nucleus induce an increase of impulsivity in rats as measured by premature responding in a choice reaction time test ${ }^{34}$. High frequency stimulation (HFS) has been shown to cause a net inhibition of the target region, an effect that is clinically comparable to a lesion ${ }^{35}$, although the underlying mechanisms are probably different. The benefit of HFS above lesions is that it is reversible and adjustable. Therefore, we chose to perform electrical stimulation of the MD thalamic nucleus in rats as a behavioural model of impulsivity, in which premature responses serve as a measure of inhibitory control ${ }^{36}$. To evaluate the specificity of the site of stimulation, we stimulated the VL thalamic nucleus. DBS of the VL thalamic nucleus is known to effectively reduce various types of tremor in patients ${ }^{37}$. Case series show a subtle memory effect of $\mathrm{VL}$ thalamic $\mathrm{DBS}^{38,39}$, but to the author's knowledge changes of impulsivity were never reported. Finally, activation in the DCbN and the PFC was evaluated using c-Fos immunohistochemistry. We focussed on the cerebellar nuclei as these are the output structures of the cerebellum. c-Fos was chosen as it is an

immediate early gene which is considered to reflect neuronal activation ${ }^{40,41}$. The expression of c-Fos is maximal about two hours after administration of a stimulus, and disappears again after four to eight hours even if the stimulus is continued ${ }^{41}$. Therefore the c-Fos expression found two hours after a specific stimulus can be considered to be the direct effect of this stimulus, and this expression can be used to identify brain areas directly influenced by this stimulus ${ }^{40}$.

\section{Materials and methods}

\section{Animals}

We used male Lewis rats (12 weeks old, bred and housed at the Central Animal Facility of the Maastricht University, The Netherlands), with an average body weight of $300 \mathrm{~g}$. The rats were housed individually in standard cages with sawdust bedding in an airconditioned room (about $20^{\circ} \mathrm{C}$ ) under a 12/12-h reversed light/dark cycle. During testing ( 5 days a week), the rats were given 10-20 g of food (standard laboratory chow; Hopefarms, Woerden, The Netherlands) a day; the other 2 days they were left to rest 
and fed ad libitum. This schedule reduced their weight to approximately $90 \%$ of their free feeding weight during the week. The rats had free access to water at all times. All experiments were approved by the Animal Experiments and Ethics Committee of the Maastricht University.

\section{Surgical procedure}

Rats were randomly assigned to one of the following groups: control (no surgery; $n=8$ ), bilateral VL thalamic nucleus electrode implantation $(n=10)$, or bilateral MD thalamic nucleus electrode implantation $(n=10)$. A detailed description of the surgical procedure has been reported previously ${ }^{42}$. In brief, animals were anaesthetized throughout the entire procedure using a combination of ketamine $(90 \mathrm{mg} / \mathrm{kg})$ and xylazine $(10 \mathrm{mg} / \mathrm{kg})$ injected subcutaneously. Rats were placed in a stereotactic apparatus (Stoelting, Wood Dale, USA; model 51653). Two burr holes were made in the skull immediately above the targets to allow for insertion of electrodes in the MD thalamic nucleus (coordinates from bregma: $A P=-2.8 \mathrm{~mm}, \mathrm{ML}= \pm 0.6 \mathrm{~mm}, \mathrm{~V}=-5.0 \mathrm{~mm}$ ) or in the $\mathrm{VL}$ thalamic nucleus (coordinates from bregma: $\mathrm{AP}=-2.8 \mathrm{~mm}, \mathrm{ML}= \pm 1.5 \mathrm{~mm}, \mathrm{~V}=-6.5 \mathrm{~mm}$ ). A construction of two stainless steel electrodes (Technomed, The Netherlands), both concentric and bipolar, with a tip diameter of $50 \mu \mathrm{m}$ and a shaft diameter of $250 \mu \mathrm{m}$, was implanted in this experiment. The electrodes were fixed in position using dental cement (Heraeus Kulzer, Germany).

\section{Deep brain stimulation}

The stimulus was delivered using a World Precision Instrument (WPI, Berlin, Germany) accupulser (A310) and a stimulus isolator (A360). Real time verification of the parameters applied during stimulation was obtained for both electrodes of the bilateral construction, using a digital oscilloscope (Agilent Technologies, Agilent 54622D oscilloscope, The Netherlands). Stimulation started a few minutes before the test and lasted for the duration of each session. The rats had 12 to 72 hours without stimulation between sessions.

\section{Behavioural evaluation}

A choice reaction time task was performed as described previously ${ }^{36}$. In summary, rats were tested in Skinner chambers (inner dimensions: $40 \mathrm{~cm} \times 30 \mathrm{~cm} \times 33 \mathrm{~cm}$ ) equipped with two retractable levers with cue lights just above the levers. A food tray $(5 \mathrm{~cm} \times$ $5 \mathrm{~cm}$ and $2.5 \mathrm{~cm}$ above the grid floor) was positioned equidistant between the two levers and could be accessed by pushing a hinged panel. The levers ( $4 \mathrm{~cm}$ wide) projected $2 \mathrm{~cm}$ into the conditioning chamber and were located $6 \mathrm{~cm}$ from both sides of the food tray and $12 \mathrm{~cm}$ above the grid floor. A light and a loudspeaker were fixed in the ceiling of the conditioning chamber. The test procedure was controlled by a personal computer and the data stored on disk at the end of a session (time precision=1 ms). During a trial, the rat had to insert its nose into the central panel until 
a tone sounded. A high tone $(10 \mathrm{kHz} ; 80 \mathrm{~dB})$ required the rat to press the left lever and a low tone $(2.5 \mathrm{kHz} ; 80 \mathrm{~dB})$ required pressing of the right lever. The period (randomly chosen from $0.6 \mathrm{~s}$ to $1.5 \mathrm{~s}$, with steps of $0.1 \mathrm{~s}$ ) between nose insertion and tone was termed the hold duration. When a rat did not hold the panel for the entire hold duration, the same interval was repeated when the panel was next pushed. Depression of the lever resulted in food reward in only $50 \%$ of cases to increase the motivation of the animals for the task ${ }^{43}$. The reinforcement was given independently of the reaction time. After pushing the panel there was a lapse of ten seconds before the next trial could be started. Each session lasted $30 \mathrm{~min}$ or until the completion of 60 trials. The following parameters were analysed:

Reaction time (RT) is defined as the latency between the onset of the tone and the release of the tray panel. It is generally accepted that RT's shorter than $100 \mathrm{~ms}$ are unlikely to be true reaction times, and RT's longer than $1500 \mathrm{~ms}$ should not be considered to be task-related; therefore these $\mathrm{RT}^{\prime}$ 's were discarded ${ }^{43}$.

Motor time (MT) is defined as the latency between the release of the tray panel and the lever press. It was assumed that $\mathrm{MT}^{\prime}$ s longer than $2 \mathrm{~s}$ did not reflect 'true' motor time; these were left out of the analysis ${ }^{43}$.

Premature responses ratio ( $\mathrm{PRr}$ ) is defined as the total number of times the rat released the tray panel before the hold duration had elapsed, divided by the number of trials. After a premature response the rats had to start the same trial again by pushing the hinged panel.

Prior to surgery, the rats underwent a period of two weeks of preliminary training until the mean reaction time of performances was stabilized, after which the baseline behavioural performance was evaluated ${ }^{43}$.

\section{Experimental procedures}

Rats were allowed to recover from surgery for 1 week after which they were retrained in the Skinner boxes (modified to allow for the attachment of a stimulation cable), until behavioural parameters were stabilized. We then tested all rats during stimulation using the following DBS parameters: a pulse width of $60 \mu \mathrm{s}$, a frequency of $130 \mathrm{~Hz}$ (high frequency), and variable amplitudes of $150,30,3$, and $1 \mu \mathrm{A}$ to investigate the effect of a relatively high, medium and low current density with respect to the human condition $^{44}$. In addition all rats were tested with the stimulation cable attached but without stimulation. The order of the various amplitudes was randomized using a Latin square design in which each condition was repeated two times. Following this, the average of the 2 sessions was calculated for each behavioural parameter.

\section{Histological processing}

One week after the final behavioural experiment all rats were stimulated in their home cage for 30 minutes using a pulse width of $60 \mu \mathrm{s}$, a frequency of $130 \mathrm{~Hz}$, and an amplitude of $30 \mu \mathrm{A}$. One hour after the end of the stimulation, they were perfused 
transcardially with Tyrode $(0.1 \mathrm{M})$, followed by a fixative containing $4 \%$ paraformaldehyde, $15 \%$ picric acid, and $0.05 \%$ gluteraldehyde in $0.1 \mathrm{M}$ phosphate buffer $(\mathrm{pH}$ 7.6). The brains were removed and postfixed for $2 \mathrm{~h}$ followed by immersion in $10 \%$ sucrose at $4^{\circ} \mathrm{C}$ until saturated. The concentration of sucrose solution was increased to $20 \%$ and finally to $30 \%$ each time the brains were saturated. Brain tissue was then quickly frozen with $\mathrm{CO}_{2}$ and stored at $-80^{\circ} \mathrm{C}$.

The prefrontal regions of the brains were cut serially on a cryostat into $30 \mu \mathrm{m}$ frontal sections and collected per 10 sections in a cup for free floating staining. The cerebellum was cut serially into $10 \mu \mathrm{m}$ sections which were collected on gelatinecoated glasses. We used a previously published protocol for c-Fos immunohistochemistry ${ }^{45}$, with some adjustments for the staining of sections on glass to achieve a similar staining intensity. In summary, this staining was carried out by incubating sections with a polyclonal rabbit anti-c-Fos antibody (Santa Cruz Biotechnology Inc., Santa Cruz, CA, USA) (Free floating sections: dilution 1:20,000; incubation overnight. Sections on glass: dilution 1:600, incubation two days), followed by incubation with the secondary antibody (biotinylated donkey anti-rabbit, Jackson Immunoresearch Laboratories Inc., Westgrove, USA) (Dilution 1:400; free floating: 90 minutes incubation; on glass: overnight incubation). Subsequently, the sections were incubated with an avidin-biotin-peroxidase complex (Elite ABC-kit, Vectastatin; Burlingame, USA) for two hours. To visualize the immune complex of Horse Radish Peroxide reaction product, sections were incubated with 3,3'-diaminobenzidine tetrahydrochloride (DAB)/ Nickel Chloride $\left(\mathrm{NiCL}_{2}\right)$ solution $(5 \mathrm{ml}$ DAB solution, $5 \mathrm{ml}$ Tris/ $\mathrm{HCl}, 50 \mu \mathrm{NiCL}_{2} 8 \%$, and $3.35 \mu$ hydrogen peroxide $30 \%$ ) for ten minutes. This reaction was stopped by rinsing all sections thoroughly with TBS. The free floating sections were then mounted on gelatine-coated glasses. After dehydrating, all sections were cover-slipped with Pertex (HistolabProducts ab, Goteborg, Sweden).

Additionally, for each animal one other series of sections from the PFC was stained with standard haematoxylin-eosin (Merck, Darmstadt, Germany) to locate the electrode tips.

\section{Quantitative evaluation of c-Fos immunoreactive cells}

Systematic cell counts were performed of c-Fos immunoreactive (c-Fos-ir) cells in the prefrontal cortex and in the deep cerebellar nuclei. Our quantification method was similar to a previously reported method ${ }^{46}$, with minor modifications with respect to the magnification and the threshold value for a positive cell. These modifications were previously explained by Lamprea et al. ${ }^{46}$ and by Lim et al. ${ }^{47}$. In summary, photographs of the areas of interest were taken at 4x magnification using an Olympus DP70 camera connected to an Olympus AX70 bright-field microscope (analySIS; Imaging System, Münster, Germany). The same light intensity and threshold settings of the camera were employed for all sections. For each animal we selected four sections of the PFC (AP coordinates from bregma: 2.2 to 2.7) and four to six sections of the cerebellar nuclei (AP coordinates from bregma: -11.2 to -11.8 ) for quantification. The boundaries 
of the areas of interest (PFC (defined by the prelimbic cortex and the infralimbic cortex), DN, IN and FN) were delineated and the area was measured. The counting of the c-Fos-ir cells was performed using the image analysis program 'Image J' (version 1.38, NIH, USA). A cell was counted as c-Fos-ir if its density was $75 \%$ higher than the mean background density of that section. Artefacts in the sections were excluded from analysis to ensure the accuracy of measurements. The number of c-Fos-ir cells per square millimeter was calculated for each area of interest.

\section{Statistical analysis}

For the analysis of the behavioural data the results during stimulation were compared to the results in the off stimulation setting using the Analysis of Variance (ANOVA) repeated measures analysis. The c-Fos expression in the prefrontal cortex was analysed using ANOVA. An LSD post hoc test was used in both cases to analyse group differences in more detail. The data of the c-Fos analysis in the deep cerebellar nuclei showed a skewed distribution and were therefore analysed using the Kruskal-Wallis test. A Mann-Whitney test was used to follow up significant findings. Correlation analysis was done using a bivariate analysis and is presented as Kendall's correlation coefficient tau. Data are presented as mean \pm S.E.M. p-values lower than 0.05 were considered significant.

\section{Results}

\section{Histological evaluation of electrode localization}

In 1 rat of the VL thalamic DBS group the electrodes were out of the target (centromedian nucleus of the thalamus) and 2 rats of the VL thalamic DBS group and 3 rats of the MD thalamic DBS group lost their electrode construction during the experiment. These rats were excluded from analysis. Electrode tips were located at the level of the VL thalamic nucleus in 5 rats and in the MD thalamic nucleus in 7 rats (Figure 2.1). No histological damage was observed except for the electrode trajectory, suggesting that repeated stimulation with the current stimulation settings did not cause observable tissue damage. 

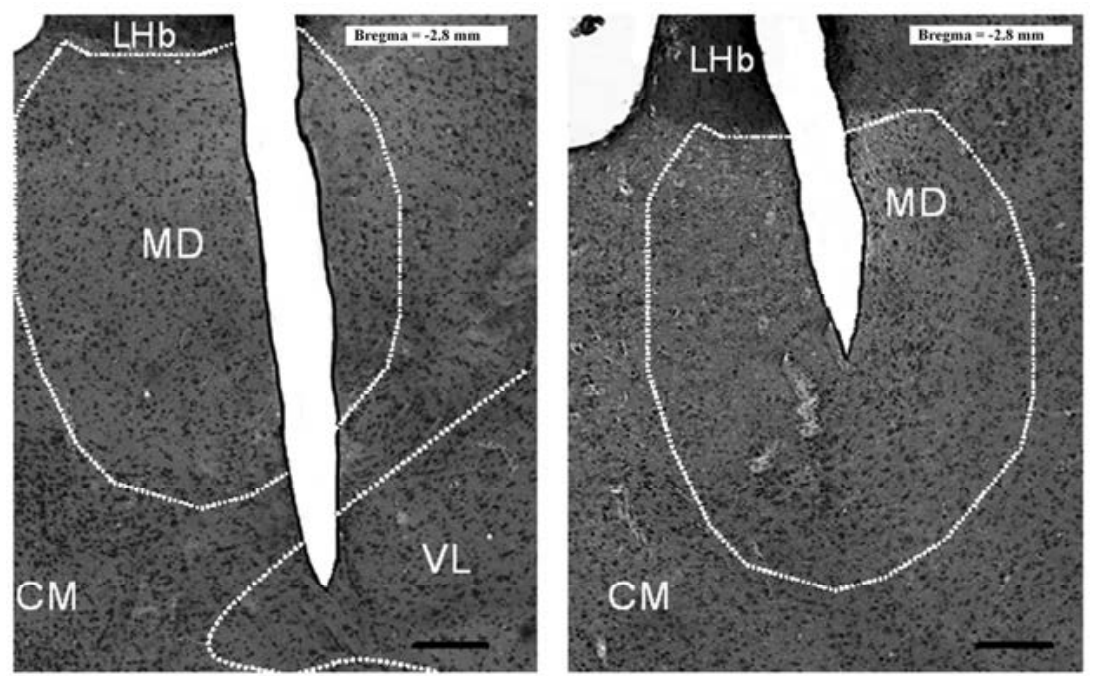

Figure 2.1 Representative low-power photomicrograph of a $30 \mu \mathrm{m}$-thick frontal section from the brains of rats with bilateral electrode implantation respectively in the ventrolateral (VL) and mediodorsal (MD) thalamic nuclei. The tips of the electrodes are situated within the respective targets. The white dotted lines show the boundaries of the structures and the dark lines delineate the boundaries of the electrodes trajectories. Scale bar=200 $\mu \mathrm{m}$. LHb: lateral habenula; CM: centromedian thalamic nucleus.

\section{Behavioural evaluation}

The behavioural parameters never exceeded the time boundaries specified in the methods section; therefore all were used for the analysis. There was a significant increase in $\mathrm{PRr}$ after DBS of the MD thalamic nucleus as compared to the off stimulation setting $\left(\mathrm{F}_{4,3}=11.061 ; \mathrm{p}<0.05\right)$ (Figure 2.2). Post hoc comparisons show an increase in $\mathrm{PRr}$ in the MD thalamic nucleus stimulated group at all stimulation parameters, however, only stimulation at $1 \mu \mathrm{A}$ caused a significant change compared to the off stimulation state. DBS of the MD thalamic nucleus had no effect on RT $\left(F_{4,3}=0.108 ; p=0.972\right)$ or $\mathrm{MT}\left(F_{4,3}=0.357 ; p=0.858\right)$ compared to the off stimulation setting. There was no significant change of $R T\left(F_{3,2}=0.869 ; p=0.574\right), M T\left(F_{3,2}=4.524\right.$; $\mathrm{p}=0.186$ ) or $\mathrm{PRr}\left(\mathrm{F}_{4,1}=92.178 ; \mathrm{p}=0.078\right)$ (Figure 2.2$)$ in the group with $\mathrm{VL}$ thalamic $\mathrm{DBS}$ as compared to the off stimulation setting. Comparison between the two groups revealed a significant difference with respect to $\mathrm{PRr}$ (stimulation*group; $\mathrm{F}_{8,24}=2.502 \mathrm{p}=0.39$ ) (Figure 2.2). 


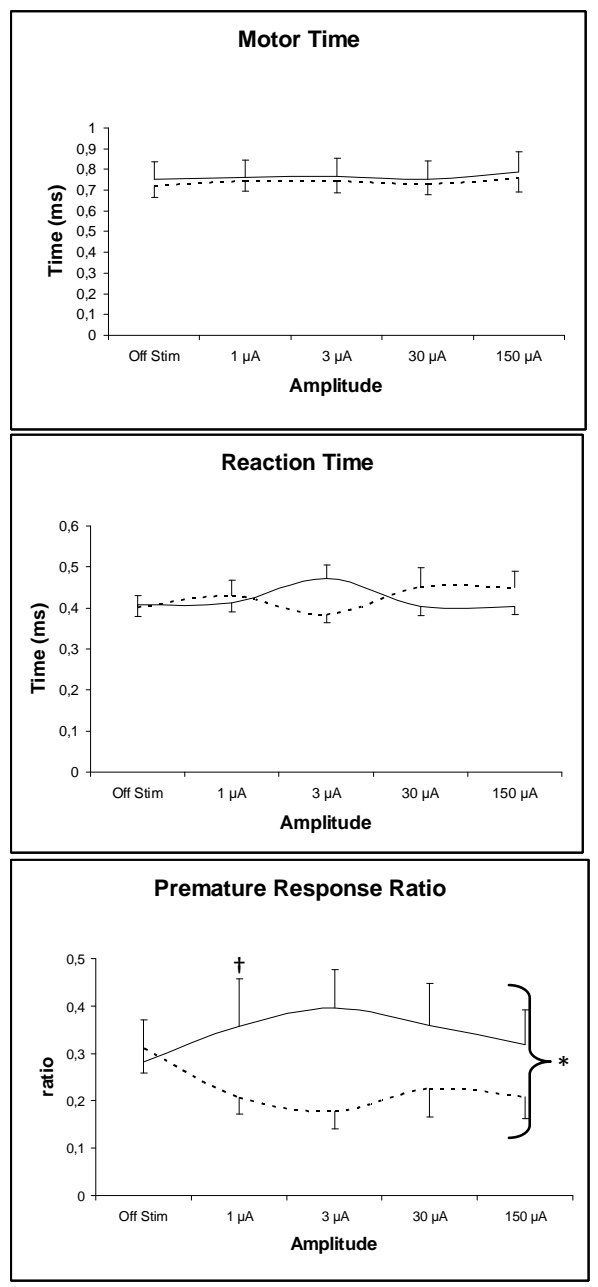

Figure 2.2 Behavioural results of rats subjected to different stimulation amplitudes using bilateral high frequency stimulation in either the MD thalamic nucleus (straight line) or the VL thalamic nucleus (dotted line). Data are shown as mean \pm SEM. A dagger $(+)$ indicates significant difference as compared to off stimulation within groups, an asterisk $\left({ }^{*}\right)$ indicates significant difference between groups. MD: mediodorsal thalamic nucleus; VL: ventrolateral thalamic nucleus.
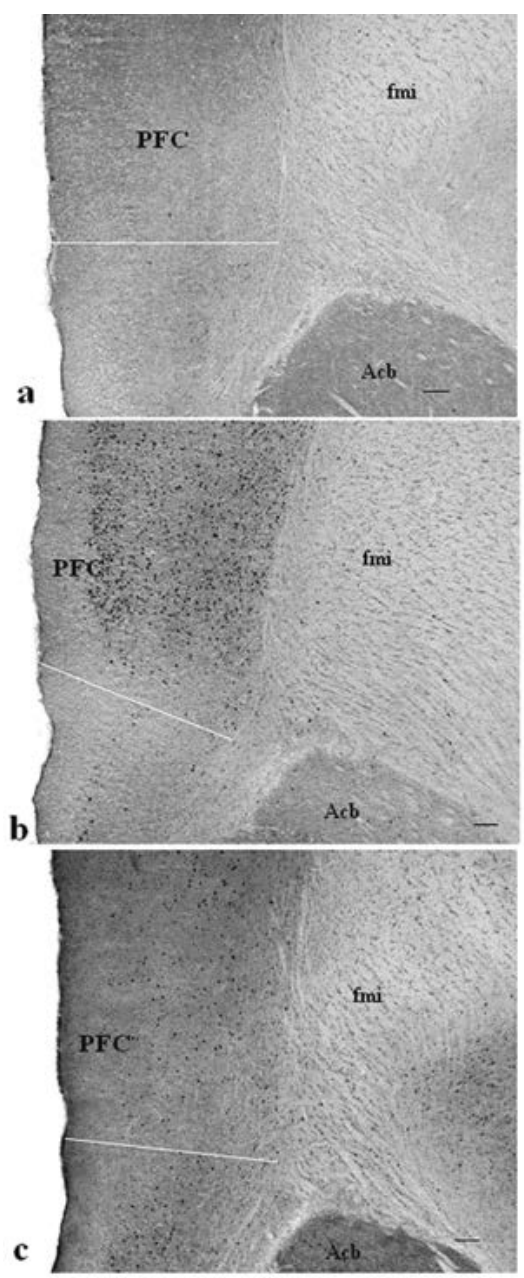

Figure 2.3 Representative low-power photomicrographs of a $30 \mu \mathrm{m}$-thick frontal section at bregma level $2.20 \mathrm{~mm}$ showing the prefrontal cortex (PFC) of a control rat (a), a rat stimulated in the MD thalamic nucleus (b) and a rat stimulated in the VL thalamic nucleus (c). The small dark dots represent c-Fos-ir cells. White lines show the lower border of the PFC. Scale bar $=300 \mu \mathrm{m}$. fmi: forceps minor of the corpus callosum; Cg1: Cingulate cortex area 1; DP: dorsopeduncular cortex. 


\section{Evaluation of the number of c-Fos-ir cells}

Since stimulation was performed bilaterally and no left/right difference was found in the number of c-Fos-ir cells, data from the left and right hemispheres were pooled. We found a significant increase in the number of c-Fos-ir cells in the prefrontal cortex $\left(F_{2,11}=4.494, p<0.05\right.$; Figure 2.3$)$ in the rats stimulated in the $M D$ thalamic nucleus $\left(21.92 \pm 5.404\right.$ c-Fos-ir cells $\left./ \mathrm{mm}^{2}\right)$ as compared to control animals $(7.603 \pm 2.913 \mathrm{c}$-Fos-ir cells $/ \mathrm{mm}^{2}$ ) and animals with DBS of the VL thalamic nucleus $(9.188 \pm 2.179 \mathrm{c}$-Fos-ir cells $/ \mathrm{mm}^{2}$ ).

In the deep cerebellar nuclei a significant difference in the number of c-Fos-ir cells in the dentate nucleus $\left(\mathrm{H}_{2}=6.057, \mathrm{p}<0.05\right)$, the interpositus nucleus $\left(\mathrm{H}_{2}=7.214, \mathrm{p}<0.05\right)$, and in the fastigial nucleus $\left(\mathrm{H}_{2}=7.419, \mathrm{p}<0.05\right)$ was found after thalamic DBS. The number of c-Fos-ir cells is lower in the MD thalamic nucleus -stimulated group compared to the VL thalamic nucleus -stimulated group and the control group in all three cerebellar nuclei (Figure 2.4 and Figure 2.5). Post-hoc analysis shows that this difference is significant between the MD thalamic nucleus -stimulated group and the control group in the dentate nucleus $(U=6.00, r=-0.59)$; in the other nuclei there is a trend which does not reach significance (IN: $U=8.00, r=-0.52 ; F N: U=7.50, r=-0.54$ ). When comparing the MD thalamic DBS group and the VL thalamic stimulated animals there is a significant difference in the interpositus nucleus $(U=1.00, r=-0.77)$ and in the fastigial nucleus $(U=1.00, r=-0.77)$; in the dentate nucleus there is a trend to significance $(U=4.00, r=-0.61)$. There is a negative relationship between the c-Fos expression in the PFC and the c-Fos expression in the $D C b N$ in the animals that were stimulated in the MD thalamic nucleus ( $\tau=-1.000, p<0.05$; Figure 2.6); a higher $c$-Fos expression in the PFC correlated with a lower c-Fos expression in the DCbN. No correlation was found in the animals with VL thalamic DBS $(\tau=0.200, p=0.624)$. All results are summarized in Table 2.1 . 

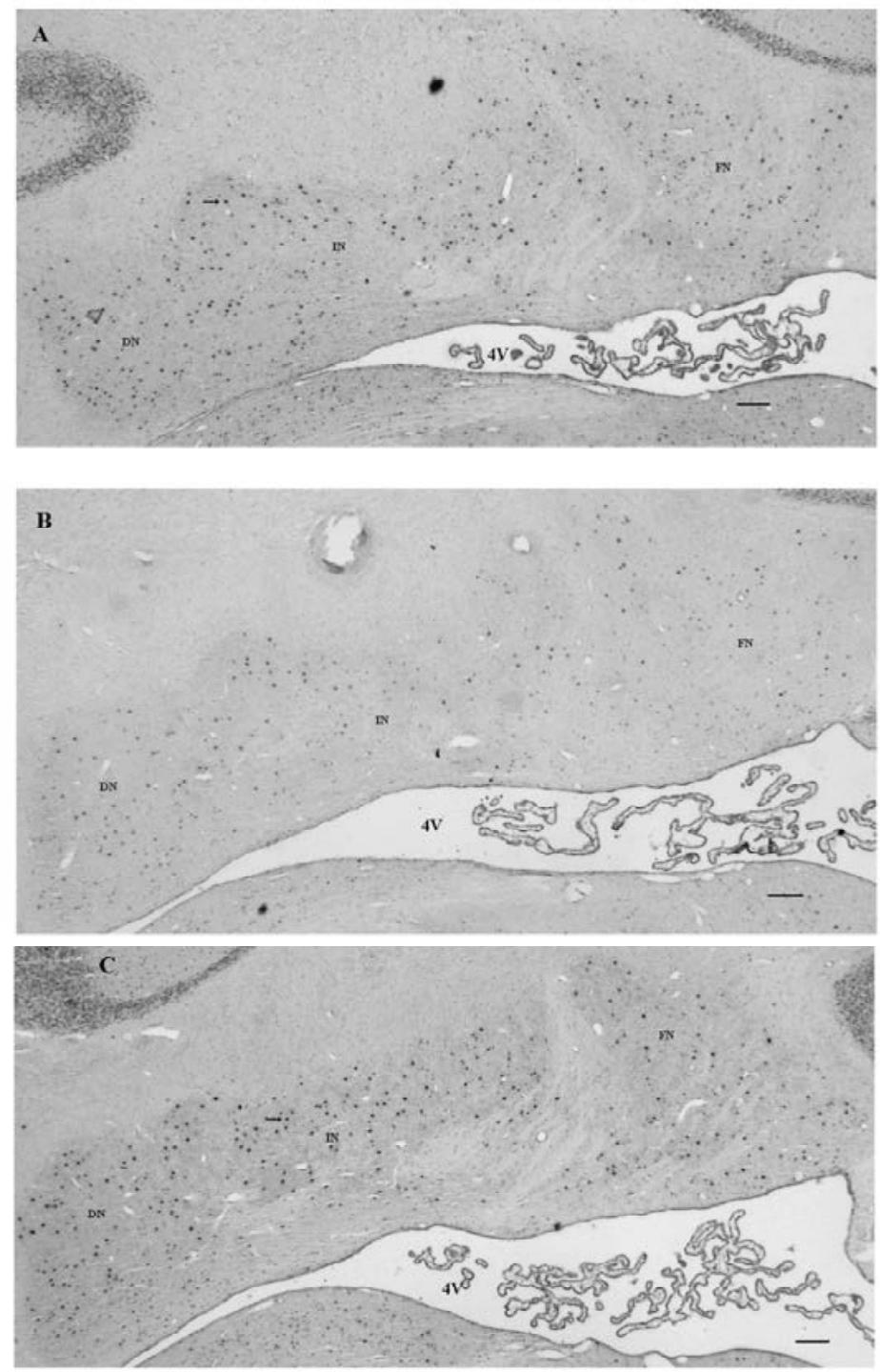

Figure 2.4: Representative low power photomicrographs of c-Fos expression in a $10 \mu \mathrm{m}$ thick frontal section of the deep cerebellar nuclei at bregma level $-11.60 \mathrm{~mm}$ in a control rat (A), a rat stimulated in the MD thalamic nucleus (B), and a rat stimulated in the $\mathrm{VL}$ thalamic nucleus (C). The arrows point to a $\mathrm{c}$-Fos immunoreactive (c-Fos-ir) cell. Cells are considered to be c-Fos-ir if their density is $75 \%$ above the background density of the section. In picture B there are no cells that meet this criterion. Scale bar $=300 \mu \mathrm{m}$. MD: mediodorsal thalamic nucleus; VL: ventrolateral thalamic nucleus; DN: dentate nucleus of the cerebellum; IN: interpositus nucleus of the cerebellum; FN: fastigial nucleus of the cerebellum; 4V: fourth ventricle. 

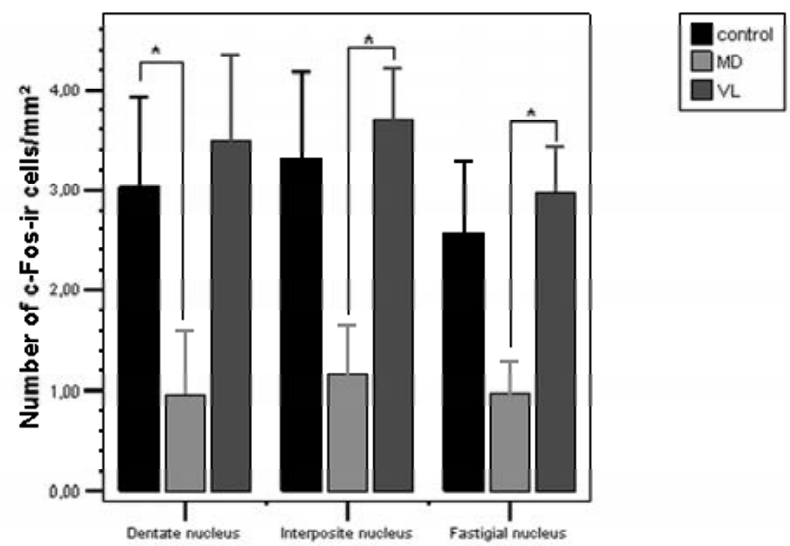

Figure 2.5 Grouped data of the c-Fos expression in the deep cerebellar nuclei. Data are represented as mean \pm S.E., showing control animals, animals with MD thalamic DBS and animals with VL thalamic DBS. An asterisk $\left({ }^{*}\right)$ indicates a significant difference between groups. MD: mediodorsal thalamic nucleus; VL: ventrolateral thalamic nucleus; DN: dentate nucleus of the cerebellum; IN: interpositus nucleus of the cerebellum; FN: fastigial nucleus of the cerebellum.

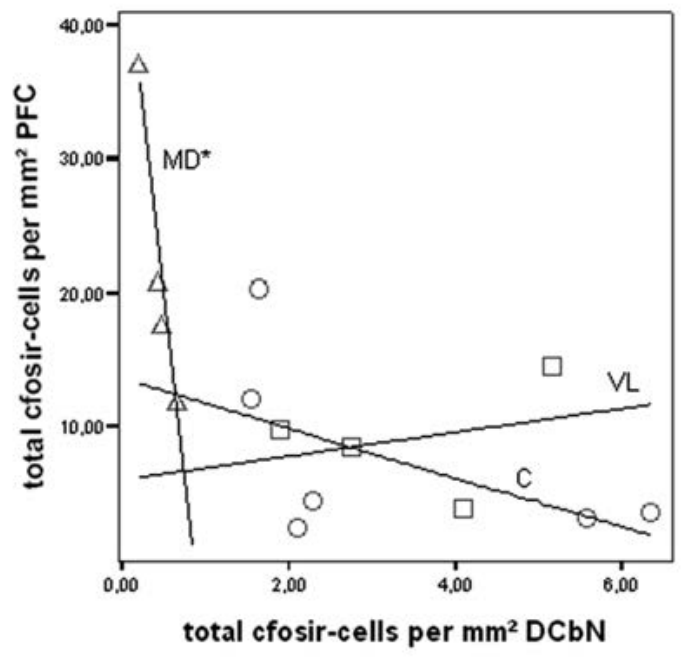

Figure 2.6 Correlation graph of c-Fos data in the DCbN and the PFC. The graph shows the amount of c-Fos-ir cells/mm2 in the DCbN on the X-axis and the amount of c-Fos-ir cells/mm2 in the PFC on the Y-axis. Circles represent control animals, triangles represent animals with MD thalamic DBS, and squares represent animals with VL thalamic DBS. Regression lines were fitted to the data to clarify the relationship between the parameters. An asterisk $(*)$ indicates a significant correlation $(\tau<0.05)$. DCbN: deep cerebellar nuclei; PFC: prefrontal cortex; MD: mediodorsal thalamic nucleus; VL: ventrolateral thalamic nucleus. 
Table 2.1 Summary of results.

\begin{tabular}{|l|c|c|c|c|c|c|c|}
\hline Parameter & \multicolumn{3}{|c|}{ Behaviour } & \multicolumn{4}{c|}{ C-Fos expression } \\
\cline { 5 - 8 } \multirow{2}{*}{$\begin{array}{l}\text { Stimulation } \\
\text { Site (below) }\end{array}$} & PRr & RT & MT & PFC & DN & IN & FN \\
\cline { 2 - 8 } & MD & $=$ & $=$ & $\uparrow *+$ & $\downarrow *$ & $\downarrow \dagger$ & $\downarrow \dagger$ \\
\hline VL & $=$ & $=$ & $=$ & $=$ & $=$ & $=$ & $=$ \\
\hline
\end{tabular}

Upward arrows $(\uparrow)$ indicate a significant increase of the measured parameter; downward arrows $(\downarrow)$ indicate a significant decrease. An equation-sign (=) indicates no significant change was found. * indicates a comparison to control; + indicates a comparison between stimulation groups. The behavioural parameters were compared to the values in the same group when the animals were not stimulated. Significant changes in PRr were only found when stimulating with $1 \mu \mathrm{A}$. No change was found in the motor parameters (RT and MT) irrespective of the stimulation parameters. PFC: prefrontal cortex; DCbN: deep cerebellar nuclei; DN: dentate nucleus of the cerebellum; IN: interposite nucleus of the cerebellum; FN: fastigial nucleus of the cerebellum; PRr: premature response ratio; RT: reaction time; MT: motor time; MD: Mediodorsal thalamic nucleus; VL: ventrolateral thalamic nucleus.

\section{Discussion}

The present study shows that there is an increase in PRr in rats after MD thalamic DBS. This can be interpreted as an increase in impulsivity as $\mathrm{PRr}$ is thought to reflect an impairment in response inhibition ${ }^{48}$. This is in line with previously described effects of lesioning of the MD thalamic nucleus on impulsive behaviour ${ }^{34}$ and confirms that, at the behavioural level, high frequency DBS mimics that lesion.

The c-Fos expression in all deep cerebellar nuclei is lower after mediodorsal thalamic nucleus stimulation as compared to animals stimulated in the ventrolateral thalamic nucleus and controls; this is significantly correlated with an increase of c-Fos expression in the prefrontal cortex as compared to controls and animals stimulated in the VL thalamic nucleus: the higher the expression in the PFC, the lower the expression in the deep cerebellar nuclei. There is no change in motor parameters of the choice reaction time test after MD thalamic stimulation, which implicates that the lower c-Fos expression we found in this group is related to the increase in impulsivity. Nabeyama and co-workers recently showed that in OCD patients the activation on f-MRI while performing a Stroop test is significantly lower in the right cerebellar tonsil compared to control patients, and that this activation increases after successful behavioural treatment ${ }^{27}$. They suggest that activation of the cerebellum and other regions related to the process of inhibition of irrelevant information might play a role in the improvement of symptoms in these patients. Others have also suggested that the cerebellum is involved in direction of selective attention, and that disruption of cerebello-thalamo-cortical connections leads to an impairment of selective attentional processes $^{49,50}$. Anatomical studies have shown that the $\mathrm{DCbN}$ project to the MD thalamic nucleus ${ }^{5,7}$. In the rat, projections from the MD thalamic nucleus to the cortex are considered to define the rat $\mathrm{PFC}^{51}$. Retrograde tracing studies have shown that the cerebellar-recipient parts of the $\mathrm{MD}$ and $\mathrm{VL}$ thalamic nuclei project to the dorsolateral 
$P F C^{8}$. The same group has shown that specific areas of the cerebellar cortex that project to the PFC also receive projections from the same part of the PFC ${ }^{4}$. They hypothesize that there are several anatomically and functionally distinct cerebellocortico-cerebellar loops. Our findings confirm that the MD thalamic nucleus is part of a cerebello-cortico-cerebellar loop through the PFC. Strick and co-workers show that the majority of the projections from the DCbN to the PFC arise from the dentate nucleus ${ }^{8}$, and that the projections from the dentate nucleus to the motor cortex originate only from $30 \%$ of the volume of the dentate nucleus ${ }^{52}$. They also show that these projections to the motor cortex arise from the dorsal part of the dentate nucleus, while the projections to the PFC arise predominantly from the ventral part of the dentate nucleus ${ }^{8,53,54}$. Based on these different anatomical connections they suggest that different parts of the dentate nucleus subserve different functions. Matano showed that there is a larger increase in the volume of the ventral part of the dentate nucleus in comparison to the dorsal part when comparing humans to great apes ${ }^{55}$. This also supports the conclusion that the ventral part of the dentate nucleus is involved in cognitive processing whereas the dorsal part subserves motor functions. In our study we found lower c-Fos expression in all cerebellar nuclei after MD thalamic DBS, without predominance in the dentate nucleus. However, if there are functionally distinct subdivisions of the dentate nucleus, an effect in one subdivision may not be evident when analysing the nucleus as a whole. Since we did not analyse the areas of the dentate nucleus separately, we cannot draw any conclusions about anatomically or functionally distinct subdivisions of the dentate nucleus. Furthermore, as discussed later, there are probably several underlying mechanisms by which deep brain stimulation can exert its influence on adjacent and remote brain structures. The exact underlying mechanisms, however, are not entirely clear. It is therefore as yet unknown whether these mechanisms pay respect to somatotopic organisation of neurons, especially when concerning remote effects. Additionally, there are authors who claim a more diffuse somatotopic organisation in the $\mathrm{DCbN}$ more consistent with a representation of muscle synergies ${ }^{56}$, in contrast to the above mentioned point-topoint somatotopy $y^{5,52,53}$.

In the animals stimulated in the VL thalamic nucleus we found no changes of behavioural parameters or c-Fos expression in the PFC or the DCbN as compared to control animals. The VL thalamic nucleus has always been seen as the primary target of cerebellar projections, and is still considered to be the main relay nucleus through which the cerebellum projects to the somatomotor cortex. VL thalamic DBS has been shown to be effective in reducing tremor in patients ${ }^{37}$ and in an animal model of tremor $^{57}$. It is therefore very interesting that we found no changes in motor parameters after DBS of the VL thalamic nucleus. This does seem to correspond to the unaltered c-Fos expression in the DCbN. Possibly the lack of effect of VL thalamic DBS in our model is due to the fact that we stimulate naïve animals. To our knowledge, studies evaluating the mechanism of thalamic DBS in animals without tremor have never analysed effects on motor parameters. VL thalamic DBS in tremor patients is not 
known to induce changes in impulsive behaviour; the lack of behavioural change in our model is in concordance with this. The absence of an effect of VL thalamic DBS on premature responding and on c-Fos expression in the DCbN supports the idea that the effects of MD thalamic stimulation we described are mediated by the prefrontal cortex and the cerebellum and are stimulation area dependent.

There is ongoing discussion about the underlying mechanism of electrical brain stimulation, despite extensive research. Nevertheless, some concepts have been generally accepted. Functionally, DBS mimics a lesion ${ }^{35}$, suggesting that the main effect of DBS is caused by inhibition of the target area ${ }^{58}$. However, the effect of DBS may be more complex: local inhibition of soma in combination with generation of an action potential in the surrounding axons ${ }^{35,59}$. Recently, the role of orthodromic versus antidromic action of DBS has gained interest. For instance, the effect that DBS of the rat nucleus accumbens has on the PFC is likely to be antidromic, whereas the effect of stimulation of the MD thalamic nucleus was more likely to be orthodromic ${ }^{60}$. We hypothesize that in our model the activation of neurons in the PFC is caused by a combination of orthodromic and antidromic stimulation since we see increased c-Fos expression in all layers of the PFC. The projections from the MD thalamic nucleus to the PFC terminate mostly in layer III of the cortex and to a lesser degree in layer I, while the reciprocate corticothalamic projections from the PFC to the MD thalamic nucleus originate mostly in layer $\mathrm{VI}^{51}$. It is postulated that, depending on the myeloarchitecture of the stimulation site, electrical stimulation leads to antidromic spikes in axons which either collide with ongoing spontaneous spikes or cause a low-frequency spike output ${ }^{61,62}$, thereby functionally suppressing the neuronal message ${ }^{63}$. Thus, MD thalamic stimulation possibly, via antidromic inhibition, resulted in a decrease in c-Fos expression in the DCbN. Alternatively, it is known that DBS can alter neurotransmitter release $^{35,63}$. Hence, orthodromic activation of the PFC may have altered the neurochemical message in the cerebellum, leading to a decreased activation of the DCbN.

In conclusion, this study supports the hypothesis that the cerebellum is involved in impulsive behaviour. High-frequent DBS of the MD thalamic nucleus has a strong effect on PRr and on c-Fos expression in the PFC and the DCbN, while VL thalamic stimulation has no significant effect on behaviour or c-Fos expression. The lack of effect of thalamic DBS on motor parameters implies that the role of the cerebellum may be a non-motor one, however, the exact nature of the place that the cerebellum has in the generation of impulsive behaviour remains to be elucidated. It is possible that the cerebellum plays a role in the process of inhibition of irrelevant information, thereby directing selective attention, and that the decreased c-fos expression we find implies a decrease of this inhibition, resulting in impulsive behaviour. 


\section{References}

1. Fine EJ, lonita CC, Lohr L. The history of the development of the cerebellar examination. Semin Neurol. 2002;22:375-84.

2. Allen GI, Tsukahara N. Cerebrocerebellar communication systems. Physiol Rev. 1974;54:957-1006.

3. Schmahmann JD, Pandya DN. Anatomic organization of the basilar pontine projections from prefrontal cortices in rhesus monkey. J Neurosci. 1997;17:438-58.

4. Kelly RM, Strick PL. Cerebellar loops with motor cortex and prefrontal cortex of a nonhuman primate. J Neurosci. 2003;23:8432-44.

5. Asanuma C, Thach WT, Jones EG. Distribution of cerebellar terminations and their relation to other afferent terminations in the ventral lateral thalamic region of the monkey. Brain Res. 1983;286:237-65.

6. Leiner HC, Leiner AL, Dow RS. Does the cerebellum contribute to mental skills? Behav Neurosci. 1986;100:443-54.

7. Yamamoto $\mathrm{T}$, Yoshida K, Yoshikawa H, Kishimoto $\mathrm{Y}, \mathrm{Oka} \mathrm{H}$. The medial dorsal nucleus is one of the thalamic relays of the cerebellocerebral responses to the frontal association cortex in the monkey: horseradish peroxidase and fluorescent dye double staining study. Brain Res. 1992 May 8;579:315-20.

8. Middleton FA, Strick PL. Cerebellar projections to the prefrontal cortex of the primate. J Neurosci. 2001;21:700-12.

9. Cavdar S, Onat FY, Yananli HR, Sehirli US, Tulay C, Saka E, Gürdal E. Cerebellar connections to the rostral reticular nucleus of the thalamus in the rat. J Anat. 2002;201:485-91.

10. Ronning C, Sundet K, Due-Tonnessen B, Lundar T, Helseth E. Persistent cognitive dysfunction secondary to cerebellar injury in patients treated for posterior fossa tumors in childhood. Pediatr Neurosurg. 2005;41:15-21.

11. Levisohn L, Cronin-Golomb A, Schmahmann JD. Neuropsychological consequences of cerebellar tumour resection in children: cerebellar cognitive affective syndrome in a paediatric population. Brain. 2000;123 ( Pt 5):1041-50.

12. Baillieux H, De Smet HJ, Paquier PF, De Deyn PP, Marien P. Cerebellar neurocognition: insights into the bottom of the brain. Clin Neurol Neurosurg. 2008;110:763-73.

13. Gordon N. The cerebellum and cognition. Eur J Paediatr Neurol. 2007;11:232-4.

14. Schmahmann JD, Sherman JC. The cerebellar cognitive affective syndrome. Brain. 1998;121: 561-79.

15. Timman D, Daum I. Cerebellar contributions to cognitive functions: A progress report after two decades of research. Cerebellum. 2007;6:159-62.

16. Catsman-Berrevoets CE, Van Dongen HR, Mulder PG, Paz y Geuze D, Paquier PF, Lequin MH. Tumour type and size are high risk factors for the syndrome of "cerebellar" mutism and subsequent dysarthria. J Neurol Neurosurg Psychiatry. 1999;67:755-7.

17. Catsman-Berrevoets $\mathrm{CE}$, van Breemen $\mathrm{M}$, van Veelen $\mathrm{ML}$, Appel IM, Lequin $\mathrm{MH}$. Supratentorial arterial ischemic stroke following cerebellar tumor resection in two children. Pediatr Neurosurg. 2005;41: 206-11.

18. Wells EM, Walsh KS, Khademian ZP, Keating RF, Packer RJ. The cerebellar mutism syndrome and its relation to cerebellar cognitive function and the cerebellar cognitive affective disorder. Dev Disabil Res Rev. 2008;14:221-8.

19. Kibby MY, Fancher JB, Markanen R, Hynd GW. A quantitative magnetic resonance imaging analysis of the cerebellar deficit hypothesis of dyslexia. J Child Neurol. 2008;23:368-80.

20. Berquin PC, Giedd JN, Jacobsen LK, Hamburger SD, Krain AL, Rapoport JL, Castellanos FX. Cerebellum in attention-deficit hyperactivity disorder; A morphometric MRI study. Neurology. 1998;50:1087-93.

21. Thomann PA, Wustenberg T, Santos VD, Bachmann S, Essig M, Schroder J. Neurological soft signs and brain morphology in first-episode schizophrenia. Psychol Med. 2008;26:1-9.

22. Andreasen NC, Pierson R. The role of the cerebellum in schizophrenia. Biol Psychiatry. 2008; 64:81-8.

23. Roth RM, Saykin AJ. Executive dysfunction in attention-deficit/hyperactivity disorder: cognitive and neuroimaging findings. Psychiatr Clin North Am. 2004;27:83-96.

24. Busatto GF, Zamignani DR, Buchpiguel CA, Garrido GE, Glabus MF, Rocha ET, Maia AF, Rosario-Campos MC, Campi Castro C, Furuie SS, Gutierrez MA, McGuire PK, Miguel EC. A voxel-based investigation of regional cerebral blood flow abnormalities in obsessive-compulsive disorder using single photon emission computed tomography (SPECT). Psychiatry Res. 2000;99:15-27. 
25. Kang DH, Kwon JS, Kim JJ, Youn T, Park HJ, Kim MS, Lee DS, Lee MC. Brain glucose metabolic changes associated with neuropsychological improvements after 4 months of treatment in patients with obsessive-compulsive disorder. Acta Psychiatr Scand. 2003;107:291-7.

26. Pujol J, Soriano-Mas C, Alonso P, Cardoner N, Menchón JM, Deus J, Vallejo J. Mapping structural brain alterations in obsessive-compulsive disorder. Arch Gen Psychiatry. 2004;61:720-30.

27. Nabeyama M, Nakagawa A, Yoshiura T, Nakao T, Nakatani E, Togao O, Yoshizato C, Yoshioka K, Tomita M, Kanba S. Functional MRI study of brain activation alterations in patients with obsessive-compulsive disorder after symptom improvement. Psychiatry Res. 2008;163:236-47.

28. Allen G, Courchesne E. Differential effects of developmental cerebellar abnormality on cognitive and motor functions in the cerebellum: an fMRI study of autism. Am J Psychiatry. 2003;160:262-73.

29. Palmen SJ, van Engeland H, Hof PR, Schmitz C. Neuropathological findings in autism. Brain. 2004;127(Pt 12):2572-83.

30. King JA, Tenney J, Rossi V, Colamussi L, Burdick S. Neural substrates underlying impulsivity. Ann N Y Acad Sci. 2003;1008:160-9.

31. Pierce K, Courchesne E. Evidence for a cerebellar role in reduced exploration and stereotyped behavior in autism. Biol Psychiatry. 2001;49:655-64.

32. Hendriksen JG, Vles JS. Neuropsychiatric disorders in males with duchenne muscular dystrophy: frequency rate of attention-deficit hyperactivity disorder (ADHD), autism spectrum disorder, and obsessive--compulsive disorder. J Child Neurol. 2008;23:477-81.

33. Cyrulnik SE, Hinton VJ. Duchenne muscular dystrophy: a cerebellar disorder? Neurosci Biobehav Rev. 2008;32:486-96.

34. Chudasama Y, Muir JL. Visual attention in the rat: a role for the prelimbic cortex and thalamic nuclei? Behav Neurosci. 2001;115:417-28.

35. Benazzouz A, Hallett M. Mechanism of action of deep brain stimulation. Neurology. 2000;55(12 Suppl 6):S13-6.

36. Temel Y, Visser-Vandewalle V, Aendekerk B, Rutten B, Tan S, Scholtissen B, Schmitz C, Blokland A, Steinbusch HW Acute and separate modulation of motor and cognitive performance in parkinsonian rats by bilateral stimulation of the subthalamic nucleus. Exp Neurol. 2005;193:43-52.

37. Lyons KE, Pahwa R. Deep brain stimulation and tremor. Neurotherapeutics. 2008;5:331-8.

38. Tröster Al, Wilkinson SB, Fields JA, Miyawaki K, Koller WC. Chronic electrical stimulation of the left ventrointermediate (Vim) thalamic nucleus for the treatment of pharmacotherapy-resistant Parkinson's disease: a differential impact on access to semantic and episodic memory? Brain Cogn. 1998;38:125-49.

39. Loher TJ, Gutbrod K, Fravi NL, Pohle T, Burgunder JM, Krauss JK. Thalamic stimulation for tremor. Subtle changes in episodic memory are related to stimulation per se and not to a microthalamotomy effect. J Neurol. 2003;250:707-13.

40. Smith MA, Banerjee S, Gold PW, Glowa J. Induction of c-fos mRNA in rat brain by conditioned and unconditioned stressors. Brain Res. 1992;578:135-41.

41. Sharp FR, Sagar SM, Hicks K, Lowenstein D, Hisanaga K. c-fos mRNA, Fos, and Fos-related antigen induction by hypertonic saline and stress. J Neurosci. 1991;11:2321-31.

42. Temel Y, Boothman L, Blokland A, Magill PJ, Steinbusch HW, Visser-Vandewalle V, Sharp T. Inhibition of 5-HT neuron activity and induction of depressive-like behavior by high-frequency stimulation of the subthalamic nucleus. Proc Natl Acad Sci U S A. 2007;104:17087-92.

43. Blokland A. Reaction time responding in rats. Neurosci Biobehav Rev. 1998 Oct;22(6):847-64.

44. Lozano AM, Dostrovsky J, Chen R, Ashby P. Deep brain stimulation for Parkinson's disease: disrupting the disruption. Lancet Neurol. 2002;1:225-31.

45. Lim LW, Temel Y, Sesia T, Vlamings R, Visser-Vandewalle V, Steinbusch HW, Blokland A. Buspirone induced acute and chronic changes of neural activation in the periaqueductal gray of rats. Neuroscience. 2008;155:164-73.

46. Lamprea MR, Cardenas FP, Vianna DM, Castilho VM, Cruz-Morales SE, Brandao ML. The distribution of fos immunoreactivity in rat brain following freezing and escape responses elicited by electrical stimulation of the inferior colliculus. Brain Res. 2002;950:186-94.

47. Lim LW, Blokland A, Visser-Vandewalle V, Vlamings R, Sesia T, Steinbusch H, Schruers K, Griez E, Temel Y. High-frequency stimulation of the dorsolateral periaqueductal gray and ventromedial hypothalamus fails to inhibit panic-like behaviour. Behav Brain Res. 2008;193:197-203. 
48. Desbonnet L, Temel Y, Visser-Vandewalle V, Blokland A, Hornikx V, Steinbusch HW. Premature responding following bilateral stimulation of the rat subthalamic nucleus is amplitude and frequency dependent. Brain Res. 2004;1008:198-204.

49. Marien P, Engelborghs S, Fabbro F, De Deyn PP. The lateralized linguistic cerebellum: a review and a new hypothesis. Brain Lang. 2001;79:580-600.

50. Courchesne E, Townsend J, Akshoomoff NA, Saitoh O, Yeung-Courchesne R, Lincoln AJ, James HE, Haas $\mathrm{RH}$, Schreibman L, Lau L. Impairment in shifting attention in autistic and cerebellar patients. Behav Neurosci. 1994;108:848-65.

51. Groenewegen HJ, Witter MP. Thalamus. In: Paxinos G, editor. The rat nervous system. Third ed: Elsevier (USA); 2004:407-53.

52. Hoover JE, Strick PL. The organization of cerebellar and basal ganglia outputs to primary motor cortex as revealed by retrograde transneuronal transport of herpes simplex virus type 1 . J Neurosci. 1999;19:1446-63.

53. Dum RP, Strick PL. An unfolded map of the cerebellar dentate nucleus and its projections to the cerebral cortex. J Neurophysiol. 2003;89:634-9.

54. Dum RP, Li C, Strick PL. Motor and nonmotor domains in the monkey dentate. Ann N Y Acad Sci. 2002;978:289-301.

55. Matano S. Brief communication: Proportions of the ventral half of the cerebellar dentate nucleus in humans and great apes. Am J Phys Anthropol. 2001;114:163-5.

56. Evrard HC, Craig AD. Retrograde analysis of the cerebellar projections to the posteroventral part of the ventral lateral thalamic nucleus in the macaque monkey. J Comp Neurol. 2008; 508:286-314.

57. Bekar L, Libionka W, Tian GF, Xu Q, Torres A, Wang X, Lovatt D, Williams E, Takano T, Schnermann J, Bakos R, Nedergaard M. Adenosine is crucial for deep brain stimulation-mediated attenuation of tremor. Nat Med. 2008;14:75-80.

58. Filali M, Hutchison WD, Palter VN, Lozano AM, Dostrovsky JO. Stimulation-induced inhibition of neuronal firing in human subthalamic nucleus. Exp Brain Res. 2004;156:274-81.

59. McIntyre CC, Savasta M, Kerkerian-Le Goff L, Vitek JL. Uncovering the mechanism(s) of action of deep brain stimulation: activation, inhibition, or both. Clin Neurophysiol. 2004;115: 1239-48.

60. McCracken CB, Grace AA. High-frequency deep brain stimulation of the nucleus accumbens region suppresses neuronal activity and selectively modulates afferent drive in rat orbitofrontal cortex in vivo. J Neurosci. 2007;27:12601-10.

61. Chomiak T, Hu B. Axonal and somatic filtering of antidromically evoked cortical excitation by simulated deep brain stimulation in rat brain. J Physiol. 2007;579(Pt 2):403-12.

62. Hammond C, Ammari R, Bioulac B, Garcia L. Latest view on the mechanism of action of deep brain stimulation. Mov Disord. 2008;23:2111-21.

63. Benabid AL, Wallace B, Mitrofanis J, Xia C, Piallat B, Fraix V, Batir A, Krack P, Pollak P, Berger F. Therapeutic electrical stimulation of the central nervous system. C R Biol. 2005;328:177-86. 



\section{Chapter}

Cerebellar nuclei are activated by high-frequency stimulation of the subthalamic nucleus

VMP Moers-Hornikx, JSH Vles, SKH Tan, K Cox, G Hoogland, HWM Steinbusch, Y Temel Neurosci Lett. 2011;496:111-5 


\section{Abstract}

The cerebellum, primarily considered a pure motor structure, is increasingly considered to play a role in behaviour and cognition. In a similar manner, there is increasing evidence that the basal ganglia are involved in non-motor processes. Recently a direct connection between the cerebellum and the basal ganglia has been shown to exist. High-frequency stimulation (HFS) of the subthalamic nucleus (STN) has become an accepted treatment in advanced Parkinson's disease (PD). We performed HFS of the STN in rats to evaluate the neuronal activation in the deep cerebellar nuclei (DCbN) using c-Fos immunohistochemistry. We found an increased c-Fos expression in the DCbN. Previously, we have shown that STN HFS in rats leads to decreased impulsive behaviour and our findings now suggest a link with increased DCbN activity. This is in line with our previous work showing that decreased $\mathrm{DCbN}$ activity is accompanied by disruptive behaviour. We suggest that the DCbN play a role in the selection of relevant information on which a behavioural response is based. The connection between the cerebellum and the basal ganglia may imply a role for the cerebellum in behavioural aspects of disorders of the basal ganglia. 


\section{Introduction}

The cerebellum was primarily considered to be predominantly involved in motor coordination and control ${ }^{1}$. The last two decades there is increasing evidence for a role of the cerebellum in cognition and behaviour. This evidence comes from tracing studies showing a projection from the cerebellum to the prefrontal cortex ${ }^{2,3}$ and clinical studies in patients with cerebellar lesions showing cognitive and behavioural disturbances $^{4-9}$. Furthermore imaging studies in patients with neuropsychiatric disorders such as schizophrenia ${ }^{10}$, attention-deficit-hyperactivity disorder ${ }^{11-13}$, and anxiety disorders ${ }^{14-18}$ show altered anatomy and activation patterns in the cerebellum compared to controls. In a similar manner, the basal ganglia have classically been regarded as being predominantly involved in motor behaviour. Results from tracing studies imply that the basal ganglia are also involved in cognitive and behavioural processing ${ }^{3}$. Clinical evidence can be found in idiopathic Parkinson's disease (PD) which is characterised by motor as well as cognitive and psychiatric symptoms ${ }^{19}$.

A central feature of the pathophysiology in PD is hyperactivity of the subthalamic nucleus (STN) caused by a loss of dopaminergic cells in the substantia nigra ${ }^{20}$. This leads to an increased activity of the output of the basal ganglia and subsequent inhibition of thalamocortical pathways. High-frequency stimulation (HFS) of the STN has become an important treatment option in advanced PD in the last three decades ${ }^{20}$. A substantial portion of patients treated with STN HFS develop cognitive problems ${ }^{21}$. Moreover, STN HFS in animal models has been shown to alter cognitive functioning ${ }^{22,23}$. Functional imaging is increasingly being used to analyse which structures are involved in PD and also to analyse the effect of STN HFS. These studies almost invariably show increased metabolism of the cerebellum in PD patients compared to control patients ${ }^{24-26}$, which can be reversed by levodopa treatment ${ }^{27}$. Studies analyzing STN HFS show decrease as well as increase of metabolism in several regions of the cerebellum during therapeutic STN $\mathrm{HFS}^{25,27-33}$. In some studies these changes in metabolism have been linked to motor improvement ${ }^{31,34}$; however, in other studies this correlation was not found ${ }^{33,35}$. Correlation with cognition or behaviour was not addressed in these studies. From an anatomical point of view, recent tracing experiments in nonhuman primates using a retrograde tracer have shown that there is a disynaptic projection from the STN to the cerebellum, and from the dentate nucleus of the cerebellum (DN) to the striatum, the input structure of the basal ganglia ${ }^{36,37}$. These findings imply that there is a closed loop between the cerebellum and the basal ganglia, which seems to be independent of cortical input ${ }^{37,38}$.

In this study we used HFS of the STN in a rat model to study the relationship of the STN with the cerebellum. Previously, we have demonstrated that HFS of the mediodorsal nucleus of the thalamus (MD) causes an increase in impulsive behaviour, which is accompanied by a decreased activation of the deep cerebellar nuclei (DCbN) ${ }^{39}$. Furthermore we have shown that in rats in which panic-like behaviour is induced by HFS of the dorsolateral periaqueductal gray (dIPAG), there is again a decreased 
activation of the $\mathrm{DCbN}^{40}$. We hypothesize that the cerebellum plays a role in the selection of relevant information to coordinate appropriate behaviour, and that deactivation of the cerebellum may lead to an increase of inappropriate behaviour. It has been shown in a rat model that HFS of the STN in naïve animals causes a decrease in impulsive behaviour ${ }^{23}$. We therefore expect an increased activation of the DCbN in the present study. To map the activation in the $D C b N$ we used c-Fos immunohistochemistry. c-Fos is an immediate early gene which is considered to reflect acute neuronal activation ${ }^{41,42}$.

\section{Materials and methods}

\section{Subjects}

Subjects were Lewis male rats ( $n=10,10$ weeks old, bred and housed at the Central Animal Facility of Maastricht University, Maastricht, The Netherlands) with an average body weight of 300 to 400 grams at the time of surgery. They were housed individually in standard cages with sawdust bedding in an air-conditioned room (about $20^{\circ} \mathrm{C}$ ) under a 12/12-h reversed light/dark cycle. Standard laboratory chow (Hopefarms, Woerden, The Netherlands) and water were available ad libitum. All experimental procedures were approved by the Animal Experiments and Ethics Committee of Maastricht University.

\section{Surgical procedure}

Rats were randomly assigned to one of two experimental groups: "sham" $(n=5)$ and "stimulation" $(n=5)$. A detailed description of the surgical procedure was reported previously ${ }^{43-45}$. In brief, animals were anaesthetized throughout the entire procedure using a combination of ketamine $(90 \mathrm{mg} / \mathrm{kg}$ s.c.) and xylazine $(10 \mathrm{mg} / \mathrm{kg}$ s.c.). They were placed in a stereotactic apparatus (Stoelting, Wood Dale, USA; model 51653). After making a burr hole in the skull all rats received bilateral implantation of electrodes at the level of the STN (coordinates from Bregma : AP -3.8, ML 2.5 and $\mathrm{V}-8,0)^{46}$. A gold-plated needle-like electrode with an inner wire of a platinum-iridium combination (Technomed, Beek, The Netherlands), with a tip diameter of $50 \mu \mathrm{m}$ and a shaft diameter of $250 \mu \mathrm{m}$ was used. In the sham group the electrodes were inserted and immediately removed. In the stimulation group the electrodes were fixed in place using dental cement (Henaeus Kulzer, Hanau, Germany).

\section{Deep brain stimulation}

Rats were allowed to recover from surgery for four days. On days five, six and seven postoperatively they received chronic stimulation during one hour each day. Stimulation was performed at $130 \mathrm{~Hz}, 60 \mu \mathrm{s}$ and $30 \mu \mathrm{A}$. These are therapeutic 
stimulation parameters ${ }^{43}$. Stimuli were delivered using a World Precision Instrument (WPI, Berlin, Germany) accupulser (A310) and a stimulus isolater (A360). Using a digital oscilloscope (Agilent 54622D oscilloscope, Agilent Technologies, The Netherlands), real time verification was obtained for the parameters applied during stimulation for both electrodes of the bilateral construction. The mode of stimulation was bipolar in all cases (inner electrode negative and outer electrode positive).

\section{Histological processing}

On post-operative day 7 the rats were sacrificed two hours after their last stimulation session. The rats were deeply anaesthetized and transcardially perfused with Tyrode $(0,1 \mathrm{M})$ and fixative containing $4 \%$ paraformaldehyde, $15 \%$ picric acid and $0.05 \%$ glutaraldehyde in $0.1 \mathrm{M}$ phosphate buffer $(\mathrm{pH} 7.6)$. Brains were removed and postfixed for $2 \mathrm{~h}$ followed by overnight immersion in $15 \%$ sucrose at $4^{\circ} \mathrm{C}$. Brain tissue was then quickly frozen with $\mathrm{CO} 2$ and stored at $-80^{\circ} \mathrm{C}$. Subsequently, the cerebelli were cut serially on a cryostat into $10 \mu \mathrm{m}$ frontal sections. These were collected on gelatinecoated glasses and stored at $-20^{\circ} \mathrm{C}$. We used a previously published protocol for c-Fos immuno-histochemistry ${ }^{39}$. In brief, sections were incubated for two days with the primary antibody (polyclonal Rabbit anti c-Fos; sc-52; Santa Cruz Biotechnology Inc., Santa Cruz, CA, USA). Subsequently sections were incubated overnight with the secondary antibody, followed by incubation with avidin-biotin-peroxidase complex for 2 hours. To visualize the immune complex sections were incubated with 3,3-diaminobenzidine (DAB) solution for 10 minutes. After dehydrating, all sections were coverslipped with Pertex (HistolabProducts ab, Goteborg, Sweden). A series of parallel sections was Nissl-stained to facilitate correct identification of the cerebellar nuclei. Additionally, for each animal the area of the STN was cut into $30 \mu \mathrm{m}$ sections and stained with standard haematoxylin-eosin (Merck, Darmstadt, Germany) staining to locate the electrode tips.

\section{Quantitative evaluation of c-Fos positive cells}

Systematic cell counts of c-Fos immunoreactive (c-Fos-ir) cells were performed in the $\mathrm{DCbN}$ as previously described ${ }^{39}$. For each animal four to ten sections with cerebellar nuclei (coordinates from Bregma AP -11.2 to -11.8) were analysed. In ImageJ (version 1.41, NIH, USA) the nuclei were delineated, the delineated area and the average grey value for each nucleus were measured. C-Fos-ir cells were counted in a semi-automatic fashion. We considered a cell to be c-Fos positive if its density was $65 \%$ higher than the average gray value of that nucleus. Artefacts in the sections were excluded from the delineations to ensure accuracy of the measurements. The number of c-Fos-ir cells per square millimeter was calculated for each nucleus. 


\section{Statistical analysis}

The data of the c-Fos-ir cell density showed a non-normal distribution in KolmogorovSmirnov and Shapiro-Wilk tests. C-Fos expression in the DCbN was therefore analysed with the non-parametric Mann-Whitney tests.

\section{Results and discussion}

In the present study we performed high frequency stimulation of the subthalamic nucleus to analyse the relationship of the STN with the cerebellum. In all animals included in this study, the electrode tips were positioned correctly in the STN (Figure 3.1). No histological damage was observed except in the electrode trajectory, suggesting that repeated stimulation did not cause observable tissue damage. Our hypothesis was that there would be increased activation in the deep cerebellar nuclei of stimulated animals compared to controls. We found an increase in c-Fos expression in the rats stimulated in the STN compared to the sham-group in the right DN $(p<0,001)$, right interpositus nucleus (IN) $(p<0,05)$ and the right fastigial nucleus (FN) $(p<0,05)$ (Figures 3.2 and 3.3). In the left nuclei there was a trend towards a higher density in the stimulated rats, however, this did not reach significance. Both the STN and the cerebellum have motor functions as well as cognitive and limbic functions. In previous studies, we performed STN HFS and found no effects on motor function but a decrease in impulsive behaviour in naïve rats ${ }^{23}$. In a rat model of Parkinson's disease using 6-OHDA-infusion in the striatum, these animals showed worsened motor function and an increase in impulsive behaviour, both of which were reversed by bilateral STN HFS ${ }^{43}$. This suggests that the increase of c-Fos expression in the DCbN is accompanied by improved cognitive functioning during STN HFS. We have previously shown that increased impulsivity caused by HFS of the MD thalamic nucleus is accompanied by a decrease of c-Fos expression in the $\mathrm{DCbN}^{39}$. In another study we showed a decrease in c-Fos expression in the DCbN of rats with dIPAG HFS induced panic-like behaviour ${ }^{40}$. Altogether these results suggest a role for the cerebellum in non-motor behaviour, in which an increase of abnormal behaviour seems to be related to a decrease in activity of the $\mathrm{DCbN}$ and in which a decrease of abnormal behaviour is associated to an increase in DCbN activity. Interestingly, in contrast to the results of our previous behavioural studies in rats ${ }^{23,43}$, PD patients treated with STN HFS often show an increase of impulsive behaviour [for review see Temel et al. $^{22}$ ]. On the other hand, several studies in PD patients treated with STN HFS show a decrease in obsessive compulsive behaviour ${ }^{47,48}$. 


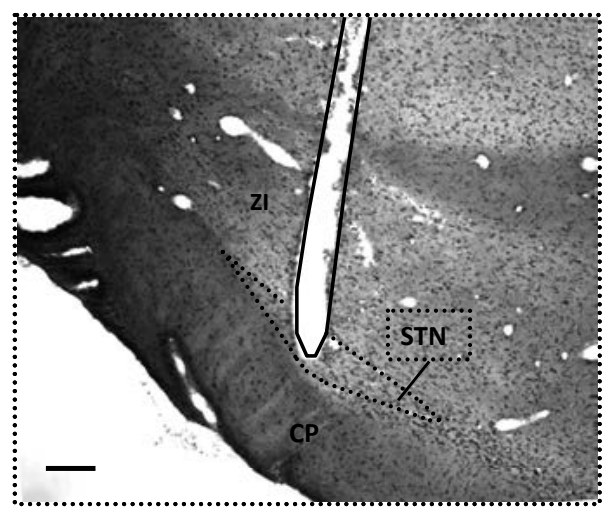

Figure 3.1 Representative low power photomicrograph of a $30 \mu \mathrm{m}$-thick frontal section of the brain of a rat subjected to stereotactic implantation of a concentric bipolar electrode in the subthalamic nucleus (STN) (scale bar $=400 \mu \mathrm{m}$ ) CP: cerebral peduncle; ZI: zona incerta.
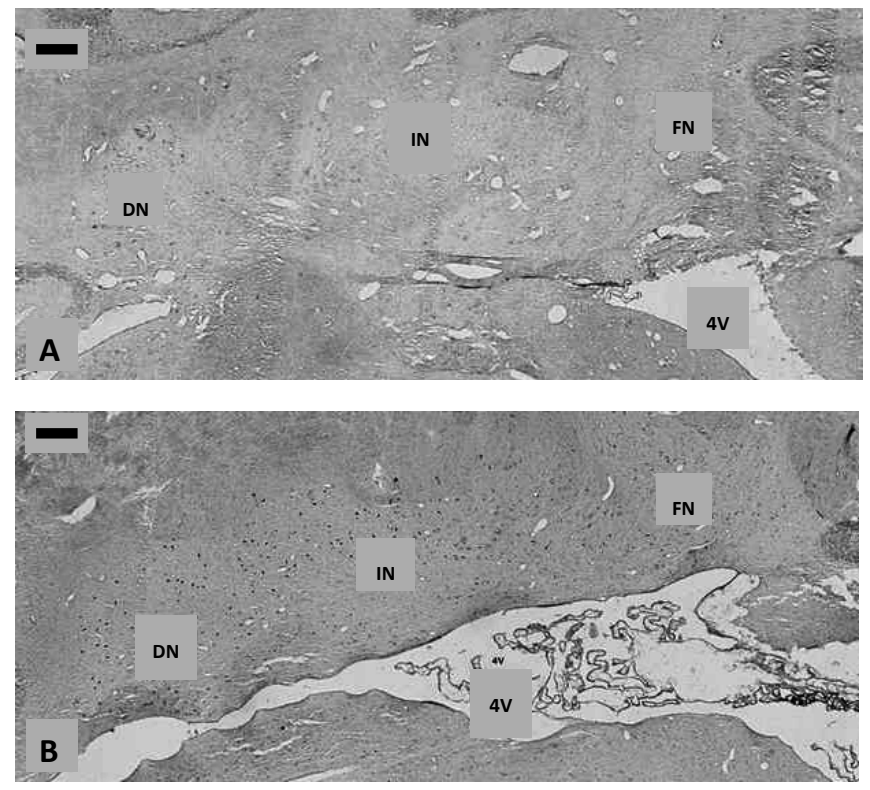

Figure 3.2 Representative low power photomicrographs of a $10 \mu \mathrm{m}$-thick horizontal section of the deep cerebellar nuclei (DCbN) at bregma $-11.60 \mathrm{~mm}$ in a sham operated animal $(\mathrm{A})$ and an animal stimulated in the subthalamic nucleus (STN) (B). Dark-gray cells represent c-Fos-immunoreactive (c-Fos-ir) cells. Note the higher density of c-Fos-ir cells in the DCbN of the animal stimulated in the STN. Scale bar $=250 \mu \mathrm{m}$ Abbreviations: DN: Dentate Nucleus, IN: Interpositus nucleus, FN: Fastigial Nucleus, 4V: fourth ventricle. 


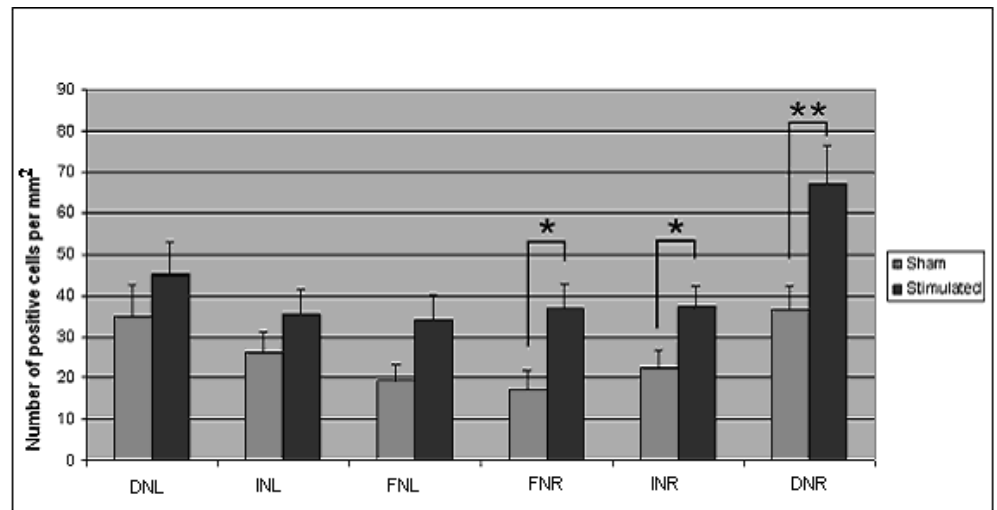

Figure 3.3 Density of c-Fos-ir cells in the deep cerebellar nuclei. Data are represented as means plus S.E.M. showing sham operated animals and animals stimulated in the subthalamic nucleus (STN) The asterisk $\left({ }^{*}\right)$ indicates a significant difference between the groups $(p<0,05)$; a double asterisk $\left({ }^{* *}\right)$ indicates $p<0,001$. Note the significantly higher density of c-Fos-ir neurons in the groups with animals stimulated in the STN. DNL: Dentate nucleus, left side, INL: Interpositus nucleus left side, FNL: Fastigial nucleus, left side, FNR: Fastigial nucleus right side, INR: Interpositus nucleus, right side, DNR: Dentate nucleus right side.

Functional imaging studies analysing the effect of STN HFS using FDG$\mathrm{PET}^{25,27,28,31,32}$, rCBPF-PET ${ }^{29,30,33}$, ECD-SPECT ${ }^{35}$ and $\left[{ }^{15} \mathrm{O}^{\mathrm{H}} \mathrm{H}_{2} \mathrm{O}-\mathrm{PET}^{49}\right.$ show increased as well as decreased metabolic activity in the cerebellum during STN HFS. These changes were sometimes correlated to motor improvement ${ }^{31,34}$, although other groups found no correlation $^{33,35}$. Correlation with cognitive or behavioural parameters was never analysed. Stefurak and coworkers describe a patiënt with bilateral STN-HFS who showed motor improvement and increased activity of the cerebellum on fMRI when the left STN was stimulated ${ }^{34}$. When the right electrode, which was in the zona incerta, was stimulated she suffered from acute depressive dysphoria. There was no change in activity of the cerebellum when this electrode was stimulated.

These results imply that there may be a functional connection between the STN and the cerebellum. Aravamuthan and coworkers used probabilistic diffusion tractography (PDT) to analyse the topographical connections of the STN in humans ${ }^{50}$. They found an anatomical connection between the STN and the mid-cerebellum. However, this technique does not give information about the direction or the excitatory or inhibitory nature of these connections. Recent tracing experiments in nonhuman primates using a retrograde tracer that is transported transneuronally show that there is a bisynaptic connection from the STN to the contralateral cerebellar cortex, and from the $\mathrm{DCbN}$ to the striatum, the input stage of basal ganglia processing $^{36-38}$. These connections are topographically organised ${ }^{36,38}$, and seem to be involved in integrating basal ganglia and cerebellar functions in motor as well as 
nonmotor processing ${ }^{37,38}$. It has already been shown that both the basal ganglia and the cerebellum form anatomically and functionally separate closed loops with several cortical areas ${ }^{2,3}$. It was postulated that interaction between these loops only takes place at the level of the cortex. However, the current findings imply that the cerebellum and the basal ganglia may be linked together to form a functional network which is independent from the cortex ${ }^{37,38}$.

We found a significant effect only in the right DCbN. Although a technical problem such as a slight difference in electrode positioning between the right and the left STN cannot be ruled out, it is possible that this is related to the different functions of the cerebellar hemispheres. Several studies have shown that injury to the cerebellum in preterm infants as well as adults leads to neuropsychological deficits, regardless of which cerebellar hemisphere is injured ${ }^{4,9}$. However, there is a difference in the type of neuropsychological deficits that are seen: right-sided lesions were associated with deficits of logical reasoning and language processing; functions typically ascribed to the left cerebral hemisphere ${ }^{9}$. In contrast, left-sided cerebellar lesions were associated with deficits in right-hemispheric functions including attentional and visuospatial skills ${ }^{9}$. Behavioural and affective disturbances were seen almost exclusively in patients with right-sided cerebellar lesions ${ }^{9}$.

\section{Conclusion}

The present study adds to the evidence that there is a functional connection between the cerebellum and the basal ganglia, and that this connection may play a role in nonmotor as well as in motor behaviour. STN HFS leads to an increased activation of the DCbN. Previous research has shown that STN HFS in naïve rats leads to a decrease of impulsive behaviour; furthermore we have previously shown that impulsive behaviour as well as panic-like behaviour is accompanied by de-activation of the DCbN. The DCbN may therefore play a role in the selection of relevant information on which a behavioural response is based. The connection between the cerebellum and the basal ganglia further implies a role for the cerebellum in behavioural aspects of basal ganglia disorders. 


\section{References}

1. Fine EJ, lonita CC, Lohr L. The history of the development of the cerebellar examination. Semin Neurol. 2002;22:375-84.

2. Kelly RM, Strick PL. Cerebellar loops with motor cortex and prefrontal cortex of a nonhuman primate. J Neurosci. 2003;23:8432-44.

3. Middleton FA, Strick PL. Basal ganglia and cerebellar loops: motor and cognitive circuits. Brain Res Rev. 2000;31:236-50.

4. Limperopoulos C, Bassan H, Gauvreau K, Robertson RL Jr, Sullivan NR, Benson CB, Avery L, Stewart J, Soul JS, Ringer SA, Volpe JJ, duPlessis AJ. Does cerebellar injury in premature infants contribute to the high prevalence of long-term cognitive, learning, and behavioral disability in survivors? Pediatrics. 2007;120:584-93.

5. Levisohn L, Cronin-Golomb A, Schmahmann JD. Neuropsychological consequences of cerebellar tumour resection in children: cerebellar cognitive affective syndrome in a paediatric population. Brain. 2000;123 ( Pt 5):1041-50.

6. Schmahmann JD, Sherman JC. The cerebellar cognitive affective syndrome. Brain. 1998; 121:561-79.

7. Catsman-Berrevoets CE, Van Dongen HR, Mulder PG, Paz y Geuze D, Paquier PF, Lequin MH. Tumour type and size are high risk factors for the syndrome of "cerebellar" mutism and subsequent dysarthria. J Neurol Neurosurg Psychiatry. 1999;67:755-7.

8. Wells EM, Walsh KS, Khademian ZP, Keating RF, Packer RJ. The cerebellar mutism syndrome and its relation to cerebellar cognitive function and the cerebellar cognitive affective disorder. Dev Disabil Res Rev. 2008;14:221-8.

9. Baillieux H, De Smet HJ, Dobbeleir A, Paquier PF, De Deyn PP, Marien P. Cognitive and affective disturbances following focal cerebellar damage in adults: a neuropsychological and SPECT study. Cortex. 2010;46:869-79.

10. Andreasen NC, Pierson R. The role of the cerebellum in schizophrenia. Biol Psychiatry. 2008; 64:81-8.

11. Berquin PC, Giedd JN, Jacobsen LK, Hamburger SD, Krain AL, Rapoport JL, Castellanos FX. Cerebellum in attention-deficit hyperactivity disorder; A morphometric MRI study. Neurology. 1998;50:1087-93.

12. Kibby MY, Fancher JB, Markanen R, Hynd GW. A quantitative magnetic resonance imaging analysis of the cerebellar deficit hypothesis of dyslexia. J Child Neurol. 2008;23:368-80.

13. Roth RM, Saykin AJ. Executive dysfunction in attention-deficit/hyperactivity disorder: cognitive and neuroimaging findings. Psychiatr Clin North Am. 2004;27:83-96.

14. Kilts CD, Kelsey JE, Knight B, et al. The neural correlates of social anxiety disorder and response to pharmacotherapy. Neuropsychopharmacology. 2006;31:2243-53.

15. Busatto GF, Zamignani DR, Buchpiguel CA, Garrido GE, Glabus MF, Rocha ET, Maia AF, Rosario-Campos MC, Campi Castro C, Furuie SS, Gutierrez MA, McGuire PK, Miguel EC. A voxel-based investigation of regional cerebral blood flow abnormalities in obsessive-compulsive disorder using single photon emission computed tomography (SPECT). Psychiatry Res. 2000;99:15-27.

16. Nabeyama M, Nakagawa A, Yoshiura T, Nakao T, Nakatani E, Togao O, Yoshizato C, Yoshioka K, Tomita M, Kanba S. Functional MRI study of brain activation alterations in patients with obsessive-compulsive disorder after symptom improvement. Psychiatry Res. 2008;163:236-47.

17. Sakai $Y$, Kumano H, Nishikawa M, Sakano $Y$, Kaiya H, Imabayashi E, Ohnishi T, Matsuda H, Yasuda A, Sato A, Diksic M, Kuboki T. Cerebral glucose metabolism associated with a fear network in panic disorder. Neuroreport. 2005;16:927-31.

18. Warwick JM, Carey P, Jordaan GP, Dupont $P$, Stein DJ. Resting brain perfusion in social anxiety disorder: a voxel-wise whole brain comparison with healthy control subjects. Prog Neuropsychopharmacol Biol Psychiatry. 2008;32:1251-6.

19. Chaudhuri KR, Schapira AH. Non-motor symptoms of Parkinson's disease: dopaminergic pathophysiology and treatment. Lancet Neurol. 2009;8:464-74.

20. Lozano AM, Dostrovsky J, Chen R, Ashby P. Deep brain stimulation for Parkinson's disease: disrupting the disruption. Lancet Neurol. 2002;1:225-31.

21. Temel Y, Blokland A, Ackermans L, Boon P, van Kranen-Mastenbroek VH, Beuls EA, Spincemaille GH, Visser-Vandewalle V. Differential effects of subthalamic nucleus stimulation in advanced Parkinson disease on reaction time performance. Exp Brain Res. 2006;169: 389-99. 
22. Temel Y, Blokland A, Steinbusch HWM, Visser-Vandewalle V. The functional role of the subthalamic nucleus in cognitive and limbic circuits. Prog Neurobiol. 2005;76:393-413.

23. Desbonnet L, Temel Y, Visser-Vandewalle V, Blokland A, Hornikx V, Steinbusch HW. Premature responding following bilateral stimulation of the rat subthalamic nucleus is amplitude and frequency dependent. Brain Res. 2004;1008:198-204.

24. Kapitán M, Ferrando R, Diéguez E, de Medina O, Aljanati R, Ventura R, Amorin I, Salinas D, Langhain M, Gioia A, Cardoso A, Lago G, Buzó R. [Regional cerebral blood flow changes in Parkinson's disease: correlation with disease duration]. Rev Esp Med Nucl. 2009;28:114-20.

25. Hilker R, Voges J, Weisenbach S, Kalbe E, Burghaus L, Ghaemi M, Lehrke R, Koulousakis A, Herholz K, Sturm V, Heiss WD. Subthalamic nucleus stimulation restores glucose metabolism in associative and limbic cortices and in cerebellum: evidence from a FDG-PET study in advanced Parkinson's disease. J Cereb Blood Flow Metab. 2004;24:7-16.

26. Sen S, Kawaguchi A, Truong Y, Lewis MM, Huang X. Dynamic changes in cerebello-thalamo-cortical motor circuitry during progression of Parkinson's disease. Neuroscience. 2010; 166:712-9.

27. Asanuma K, Tang C, Ma Y, Dhawan V, Mattis P, Edwards C, Kaplitt MG, Feigin A, Eidelberg D. Network modulation in the treatment of Parkinson's disease. Brain. 2006;129:2667-78.

28. Hilker R, Voges J, Thiel A, Ghaemi M, Herholz K, Sturm V, Heiss WD. Deep brain stimulation of the subthalamic nucleus versus levodopa challenge in Parkinson's disease: measuring the on- and offconditions with FDG-PET. J Neural Transm. 2002;109:1257-64.

29. Grafton ST, Turner RS, Desmurget M, Bakay R, Delong M, Vitek J, Crutcher M. Normalizing motorrelated brain activity; Subthalamic nucleus stimulation in Parkinson disease. Neurology. 2006;66: 1192-9.

30. Geday J, Ostergaard K, Johnsen E, Gjedde A. STN-stimulation in Parkinson's disease restores striatal inhibition of thalamocortical projection. Human Brain Mapping. 2009;30: 112-21.

31. Trost M, Su S, Su P, Yen RF, Tseng HM, Barnes A, Ma Y, Eidelberg D. Network modulation by the subthalamic nucleus in the treatment of Parkinson's disease. Neuroimage. 2006;31:301-7.

32. Wang J, Ma Y, Huang Z, Sun B, Guan Y, Zuo C. Modulation of metabolic brain function by bilateral subthalamic nucleus stimulation in the treatment of Parkinson's disease. J Neurol. 2010;257:72-8.

33. Sestini S, Ramat S, Formiconi AR, Ammanati F, Sorbi S, Pupi A. Brain Networks Underlying the Clinical Effects of Long-Term Subthalamic Stimulation for Parkinon's Disease: A 4-Year Follow-up Study with rCBF SPECT. J Nucl Med. 2005;46:1444-54.

34. Stefurak T, Mikulis D, Mayberg H, Lang AE, Hevenor S, Pahapill P, Saint-Cyr J, Lozano A. Deep brain stimulation for Parkinson's disease dissociates mood and motor circuits: a functional MRI case study. Mov Disord. 2003;18:1508-16.

35. Cilia R, Marotta G, Landi A, Isaias IU, Mariani CB, Vergani F, Benti R, Sganzerla E, Pezzoli G, Antonini A. Clinical and cerebral activity changes induced by subthalamic nucleus stimulation in advanced Parkinson's disease: A prospective case-control study. Clin Neurol Neurosurg. 2009;111:140-6.

36. Hoshi E, Tremblay L, Feger J, Carras PL, Strick PL. The cerebellum communicates with the basal ganglia. Nat Neurosci. 2005;8:1491-3.

37. Bostan AC, Strick PL. The cerebellum and basal ganglia are interconnected. Neuropsychol Rev. 2010;20:261-70.

38. Bostan AC, Dum RP, Strick PL. The basal ganglia communicate with the cerebellum. Proc Natl Acad Sci U S A. 2010;107:8452-6.

39. Moers-Hornikx VM, Sesia T, Basar K, et al. Cerebellar nuclei are involved in impulsive behaviour. Behav Brain Res. 2009;203:256-63.

40. Moers-Hornikx VM, Vles JS, Lim LW, Ayyildiz M, Kaplan S, Gavilanes AW, Hoogland G, Steinbusch HW, Temel Y. Periaqueductal grey stimulation induced panic-like behaviour is accompanied by deactivation of the deep cerebellar nuclei. Cerebellum. 2011;10:61-9.

41. Smith MA, Banerjee S, Gold PW, Glowa J. Induction of c-fos mRNA in rat brain by conditioned and unconditioned stressors. Brain Res. 1992;578:135-41.

42. Sharp FR, Sagar SM, Hicks K, Lowenstein D, Hisanaga K. c-fos mRNA, Fos, and Fos-related antigen induction by hypertonic saline and stress. J Neurosci. 1991;11:2321-31.

43. Temel Y, Visser-Vandewalle V, Aendekerk B, Rutten B, Tan S, Scholtissen B, Schmitz C, Blokland A, Steinbusch HW. Acute and separate modulation of motor and cognitive performance in parkinsonian rats by bilateral stimulation of the subthalamic nucleus. Exp Neurol. 2005;193:43-52. 
44. Temel Y, Boothman L, Blokland A, Magill PJ, Steinbusch HW, Visser-Vandewalle V, Sharp T. Inhibition of 5-HT neuron activity and induction of depressive-like behavior by high-frequency stimulation of the subthalamic nucleus. Proc Natl Acad Sci U S A. 2007;104:17087-92.

45. Tan SKh, Vlamings R, Lim L, Sesia T, Janssen ML, Steinbusch HW, Visser-Vandewalle V, Temel Y. Experimental deep brain stimulation in animal models. Neurosurgery. 2010;67:1073-9; discussion 80.

46. Paxinos G, Watson C. The Rat Brain in Stereotaxic Coordinates. Fourth ed. New York: Academic Press; 1998.

47. Mallet L, Mesnage V, Houeto JL, Pelissolo A, Yelnik J, Behar C, Gargiulo M, Welter ML, Bonnet AM, Pillon B, Cornu P, Dormont D, Pidoux B, Allilaire JF, Agid Y. Compulsions, Parkinson's disease, and stimulation. Lancet. 2002;360:1302-4.

48. Alegret $M$, Junqué $C$, Valldeoriola $F$, Vendrell $P$, Pilleri $M$, Rumià J, Tolosa $E$. Effects of bilateral subthalamic stimulation on cognitive function in Parkinson disease. Arch Neurol. 2001;58:1223-7.

49. Payoux P, Remy P, Damier P, Miloudi M, Loubinoux I, Pidoux B, Gaura V, Rascol O, Samson Y, Agid Y. Subthalamic nucleus stimulation reduces abnormal motor cortical activity in Parkinson disease. Arch Neurol. 2004;61:1307-13.

50. Aravamuthan BR, Muthusamy KA, Stein JF, Aziz TZ, Johansen-Berg H. Topography of cortical and subcortical connections of the human pedunculopontine and subthalamic nuclei. Neuroimage. 2007;37:694-705. 


\section{Chapter 4}

Periaqueductal grey stimulation induced panic-like behaviour is accompanied by deactivation of the deep cerebellar nuclei

VMP Moers-Hornikx, JSH Vles, LW Lim, M Ayyildiz, S Kaplan, AWD Gavilanes, G Hoogland, HWM Steinbusch, Y Temel 


\section{Abstract}

Until recently the cerebellum was primarily considered to be a structure involved in motor behaviour. New anatomical and clinical evidence has shown that the cerebellum is also involved in higher cognitive functions and non-motor behavioural changes. Functional imaging in patients with anxiety disorders and in cholecystokinin tetrapeptide (CCK-4) induced panic-attacks show activation changes in the cerebellum. Deep brain stimulation (DBS) of the dorsolateral periaqueductal grey (dIPAG) and the ventromedial hypothalamus (VMH) in rats has been shown to induce escape behaviour, which mimics a panic attack in humans. We used this animal model to study the neuronal activation in the deep cerebellar nuclei (DCbN) using c-Fos immunohistochemistry. c-Fos expression in the $\mathrm{DCbN}$ decreased significantly after inducing escape behaviour by stimulation of the dIPAG and the VMH, indicating that the DCbN were deactivated. This study demonstrates that the $\mathrm{DCbN}$ are directly or indirectly involved in panic attacks. We suggest that the cerebellum plays a role in the selection of relevant information, and that deactivation of the cerebellar nuclei is required to allow inappropriate behaviour to occur, such as panic attacks. 


\section{Introduction}

The cerebellum has long been considered to be predominantly involved in motor coordination and control $^{1,2}$. The last two decades, however, it has become increasingly clear that the cerebellum is also involved in behaviour and cognition ${ }^{3,4}$. Schmahmann and Sherman described the cerebellar cognitive affective syndrome (CCAS) consisting of impairment of executive functions, difficulties with spatial cognition, changes of personality including disinhibited or inappropriate behaviour and language deficits ${ }^{5}$. Recent neuroanatomical and functional imaging research has pointed towards a role for the cerebellum in neuropsychiatric diseases such as autism, schizophrenia and mood and anxiety disorders ${ }^{6-11}$. With respect to anxiety disorders, Levinson described the existence of cerebellar-vestibular dysfunction in $94 \%$ of patients with various types of anxiety disorders ${ }^{12}$. De Bellis and Kuchibhatla showed that children and adolescents with post-traumatic stress disorder related to maltreatment have significantly smaller cerebelli than paediatric subjects with generalized anxiety disorder and healthy subjects $^{13}$. Several groups have shown structural and functional differences in the cerebellum of patients with anxiety disorders compared to control subjects ${ }^{14-22}$. In patients with obsessive-compulsive disorder (OCD), a disorder characterised by anxiety, an increase in grey matter was found in the anterior cerebellum ${ }^{22}$. Using functional imaging, Busatto and colleagues found an increased activation in the superior cerebellum of OCD patients compared to control subjects ${ }^{14}$. Another group showed that after treatment, OCD patients showed a decreased activation in the cerebellum compared to pretreatment scans ${ }^{15}$. In contrast, Nabeyama and coworkers found decreased activation in untreated OCD-patients compared to control subjects, which increased after succesfull treatment of these patients ${ }^{16}$. In patients with panic disorder an increased activation of the cerebellum was found when compared to control patients ${ }^{20}$, this activation decreased again after successful treatment of these patients $^{21}$. In healthy individuals in whom panic attacks were induced by cholecystokinin tetrapeptide (CCK-4) an increased activation of the cerebellum was found, especially in the vermis ${ }^{23-26}$. However, the significance of these findings is not yet clear.

Panic disorder is one of the most frequently encountered anxiety disorders ${ }^{27}$. Panic attacks consist of an acute, sometimes unexpected onset of intense fear, accompanied by a variety of physical symptoms ${ }^{27}$. Treatment usually includes behavioural and pharmacological therapies, either alone or in combination. It is thought that panic attacks may be the result of an abnormally sensitive fear network ${ }^{28}$. The periaqueductal grey (PAG) is known to be part of this fear network which further consists of the central nucleus of the amygdala, ventromedial hypothalamus (VMH), hippocampus, and other brainstem regions ${ }^{28}$. Deep brain stimulation (DBS) in rats of the dorsolateral periaqueductal grey (dIPAG) and one of its target structures, the ventromedial hypothalamus has been shown to elicit a typical behaviour consisting of vigorous running and jumping ${ }^{29}$. This behaviour, also known as escape behaviour, is 
accompanied by strong emotional and autonomic activation, and thereby mimics panic attacks in humans ${ }^{29,30}$.

In the present study, we used DBS of the dIPAG and the VMH as a model of panic attack to evaluate the involvement of the deep cerebellar nuclei (DCbN). In a previous study, we have found that increased impulsivity in rats was accompanied by a deactivation of the $\mathrm{DCbN}^{4}$. These findings suggested that the cerebellum plays a role in regulating appropriate behaviour. Based on their findings in a patient with pathological laughter and crying, Parvizi and co-workers hypothesized that the cerebellum adjusts behavioural responses to the correct situational context of a stimulus, and that when the cerebellum operates on the basis of incorrect information, this could lead to inappropriate or even chaotic behaviour ${ }^{31}$. We hypothesized that deactivation of the $\mathrm{DCbN}$ was needed for pathological behaviour to occur. In line with this, here we predicted to find again a deactivation of the $\mathrm{DCbN}$ in rats with panic-like behaviour. Panic attacks consist of an inadequate response to a stimulus. Possibly the cerebellum also plays a role in regulation of the response to these stimuli by selection of relevant information. Deactivation of the $\mathrm{DCbN}$ may then be linked to an inadequate response to a certain stimulus by disabling this selection process. To analyse activation patterns we have used c-Fos immuno-histochemistry. We focused on the DCbN as these are the output structures of the cerebellum. c-Fos was chosen as it is an immediate early gene which is considered to reflect neuronal activation ${ }^{32,33}$. The level of c-Fos protein is maximal about two hours after administration of a stimulus, and disappears again after four to eight hours even if the stimulus is continued ${ }^{32}$. Therefore, the c-Fos expression found two hours after a specific stimulus in controlled conditions can be considered to result directly from the stimulus, and this expression can be used to identify brain areas influenced by this stimulus ${ }^{33}$.

\section{Materials and methods}

\section{Animals}

The animals used were male albino Wistar rats $(n=15,12$ weeks old, bred and housed at the Central Animal Facility of the Maastricht University, The Netherlands), with an average body weight of 300-350g at the time of surgery. Rats were housed individually in standard cages with sawdust bedding in an air-conditioned room under a 12/12-h reversed light/dark cycle. Food, standard laboratory chow (Hopefarms, Woerden, the Netherlands) and water were available ad libitum. All experiments were approved by the Animal Experiments and Ethics Committee of Maastricht University.

\section{Surgical procedure}

Rats were randomly assigned to one of the following three experimental groups: control (no surgery), dIPAG DBS or VMH DBS. A detailed description of the surgical 
procedure was reported previously ${ }^{29,34}$. In brief, animals were anaesthetized throughout the entire procedure using a combination of ketamine $(90 \mathrm{mg} / \mathrm{kg})$ and xylazine $(10 \mathrm{mg} / \mathrm{kg})$ injected subcutaneously. Rats were placed in a stereotactic apparatus (Stoelting, Wood Dale, USA; model 51653). After making a burr hole, rats received implantation of a unilateral electrode at the level of the right dIPAG (coordinates from Bregma: $A P=-7.6 \mathrm{~mm}, \mathrm{ML}=0.7 \mathrm{~mm}, \mathrm{~V}=-4.8 \mathrm{~mm}$; approached with a coronal angle of 10 degree) and the right $\mathrm{VMH}$ (coordinates from Bregma: $A P=-2.5 \mathrm{~mm}$, $\mathrm{ML}=0.5 \mathrm{~mm}, \mathrm{~V}=-9.5 \mathrm{~mm}$ ). A construction of one electrode with an inner wire of a platinum-irridium combination (Technomed, Beek, the Netherlands), with a tip diameter of $50 \mu \mathrm{m}$ and a shaft diameter of $250 \mu \mathrm{m}$, was implanted in this experiment ${ }^{35}$. The electrodes were fixed in position using dental cement.

\section{Deep brain stimulation}

After a recovery period of about one week, all animals underwent a first stimulation session to determine the escape threshold. The stimulation amplitudes were gradually increased until escape behaviour was observed. At each step, stimulation duration was $15 \mathrm{~s}$ followed by a period without stimulation of $45 \mathrm{~s}$. The stimulation frequency was set at $50 \mathrm{~Hz}$ and pulse width at $0.1 \mathrm{~ms}$ based on previous experiments ${ }^{29}$. A World Precision Instruments digital stimulator (DS8000, WPI, Berlin, Germany) and a stimulus isolator (DLS100, WPI, Berlin, Germany) were used to deliver the stimuli. Real time verification of the parameters applied during stimulation was obtained using a digital oscilloscope (Agilent 54622D oscilloscope, Agilent Technologies, Amstelveen, the Netherlands). After confirming the current amplitudes necessary to evoke escape behaviour, all rats had a period of two weeks without stimulation before the final stimulation session in the open field (OF) arena was performed. Control rats were handled and placed in the OF similarly.

\section{Behavioural evaluation}

Rats were evaluated in an OF test. For more details of this test, please see Lim et al. ${ }^{29}$. In summary, rats were placed in the arena and were connected with an external stimulator through externalized leads. The stimulation started approximately $1 \mathrm{~min}$ after the rat was placed in the OF arena, using the current amplitudes previously eliciting escape behaviour. The behaviour of the rats was videotaped (Ethovision, Noldus Information Technology, Wageningen, the Netherlands).

\section{Histological processing}

Two hours after the final stimulation session, rats were deeply anesthetized and perfused transcardially with Tyrode $(0.1 \mathrm{M})$, followed by a fixative containing $4 \%$ paraformaldehyde, $15 \%$ picric acid and $0.05 \%$ gluteraldehyde in $0.1 \mathrm{M}$ phosphate buffer ( $\mathrm{pH}$ 7.6). The brains were removed and postfixed for $2 \mathrm{~h}$ followed by immersion in $20 \%$ sucrose at $4^{\circ} \mathrm{C}$ until saturated. Brain tissue was then quickly frozen with $\mathrm{CO}_{2}$ and stored 
at $-80^{\circ} \mathrm{C}$. The cerebellum was cut serially into $10-\mu \mathrm{m}$ sections which were collected on gelatine-coated glasses. We used a previously published protocol for c-Fos immunohistochemistry ${ }^{4}$. In summary, this staining was carried out by incubating sections two nights with a polyclonal rabbit anti-c-Fos antibody (Santa Cruz Biotechnology Inc., Santa Cruz, CA, USA).This was followed by overnight incubation with the secondary antibody (biotinylated donkey anti-rabbit, Jackson Immunoresearch Laboratories Inc., Westgrove, USA). Subsequently, the sections were incubated with an avidin-biotin-peroxidase complex (Elite ABC-kit, Vectastatin; Burlingame, USA) for two hours. To visualize the immune complex of horseradish peroxide reaction product, sections were incubated with 3,3'-diaminobenzidine tetrahydrochloride (DAB)/Nickel Chloride $(\mathrm{NiCl} 2)$ solution for ten minutes. After dehydrating, all sections were coverslipped with Pertex (HistolabProducts ab, Goteborg, Sweden). Additionally, for each animal, the area of the dIPAG and VMH was cut into $30 \mu \mathrm{m}$ sections and stained with standard haematoxylin-eosin (Merck, Darmstadt, Germany) staining to locate the electrode tips.

\section{Stereological quantification of c-Fos immunoreactive cells}

Stereological analysis was performed with a stereology workstation (CAST-GRIDComputer Assisted Stereological Toolbox-Olympus, Denmark). After exactly tracing boundaries of the fastigial nucleus (FN) and dentate nucleus of the cerebellum (DN) on microscopic video images displayed on a monitor, numbers of c-Fos immunoreactive (c-Fos-ir) cells in both nuclei were evaluated with the modified Abercrombie cell counting method ${ }^{36}$. All neurons of which the cell bodies gave a positive reaction within an unbiased counting frame distributed in a systematic-random fashion throughout the delineated regions were counted. Estimated numbers of neurons were calculated from the numbers of counted neurons and the corresponding sampling probability. The numerical density $\left(N_{v}\right)$ of c-Fos-ir cells in the nuclei was estimated using the formula $N_{V}=\left(N_{A} / T\right)(T /(T+D))^{36}$. In this formula, $N_{A}, T$ and $D$ are the positive cell number in the unbiased counting frame, the section thickness and the mean particle diameter, respectively. To make an acceptable biological comment on any subject one should estimate total number of cells rather than numerical density of cells in the interested region. For this reason, we estimated the volume (V) of each cerebellar nucleus by multiplying the total area of each nucleus that is seen in the whole section of cerebellum $(\Sigma A)$ with the mean section thickness $(T)$, as shown in this formula $V=\Sigma A T^{37}$. The total number of cells in a nucleus was estimated by the formula $N=N_{V} V$.

\section{Semiquantitative analysis of c-Fos-immunoreactieve cells}

Analysis of the cFos-ir cells in the interpositus nucleus of the cerebellum (IN) were performed using a previously described semiquantitative method ${ }^{4}$. In summary, photographs of the IN were taken at $4 \mathrm{X}$ magnification using an Olympus DP70 camera connected to an Olympus AX70 bright-field microscope (analySIS; Imaging System, 
Münster, Germany). The same light intensity and threshold settings of the camera were employed for all sections. For each animal we selected two sections of the IN for quantification. The boundaries of the IN were delineated and the area measured. The counting of the c-Fos-ir cells was performed using the image analysis program 'Image J' (version 1.38, NIH, USA). A cell was counted as c-Fos-ir if its density was $65 \%$ higher than the mean background density of that section. Artefacts in the sections were excluded from analysis to ensure the accuracy of measurements. The number of c-Fos-ir cells per square millimeter was calculated.

\section{Statistical analysis}

The data of the c-Fos counts were analysed using the Kruskall-Wallis test for nonparametric data. Non-parametric testing was chosen due to small group sizes. A value of $p<0.05$ was considered significant. Post hoc analysis was performed using the MannWhitney test. Since we expected a deactivation in the stimulated groups, we used a one-tailed procedure.

\section{Results}

\section{Histological evaluation of the electrode localisation}

In all animals included in this study, the electrode tips were positioned correctly in the dIPAG and VMH, respectively (Figure 4.1). No histological damage was observed except for the electrode trajectory with the standard haematoxylin-eosin staining, suggesting that stimulation with the current stimulation settings did not cause observable tissue damage.

\section{Behavioural evaluation}

The intensity of the electrical current applied to the dIPAG and VMH of the animals was based on the threshold shown to induce escape behaviour. All rats showed 'escape behaviour' characterized by rigorous and aimless running. The current amplitudes necessary for inducing escape behaviour were significantly different between rats with dIPAG- and VMH-DBS. (F's>351.13; $\mathrm{p}<0.00)$. The mean current density applied to the dIPAG to induce escape behaviour was approximately $90 \mu \mathrm{A}$ and for the $\mathrm{VMH}$ approximately $600 \mu \mathrm{A}$. 


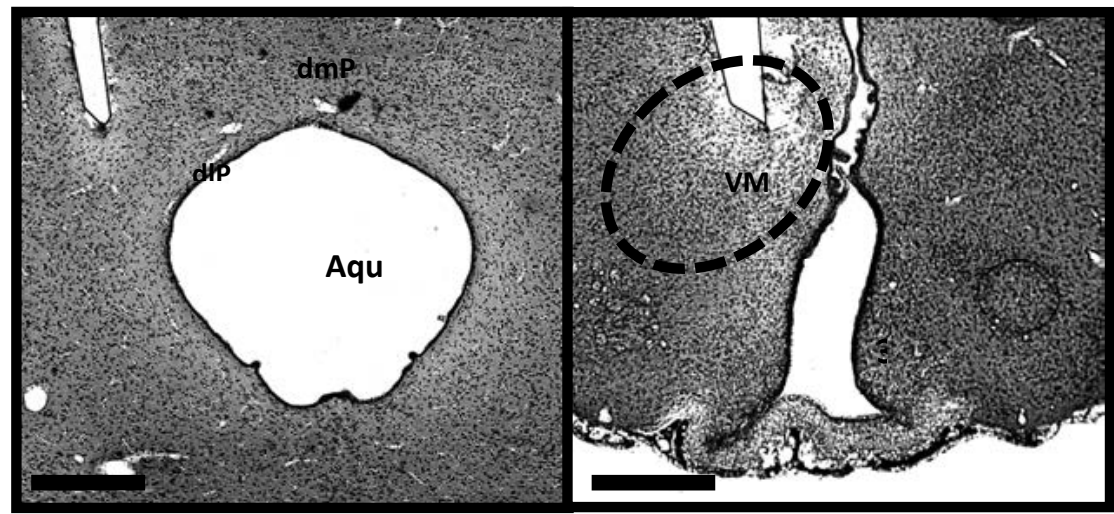

Figure 4.1 Representative low-power photomicrographs of $30 \mu \mathrm{m}$-thick frontal sections from the brain of a rat subjected to stereotactic implantation of a concentric bipolar electrode to stimulate the dIPAG (Figure 4.1a, scale bar $=250 \mu \mathrm{m}$ ) and $\mathrm{VMH}$ (Figure $4.1 \mathrm{~b}$, scale bar=500 $\mu \mathrm{m}$ ). The tips of the electrodes are situated within the respective targets. Aquad.: aqueduct of Sylvius, dmPAG: dorsomedial periaqueductal grey, dIPAG: dorsolateral periaqueductal grey, $3 \mathrm{~V}$ : third ventricle, $\mathrm{VMH}$ : ventromedial hypothalamus

\section{Evaluation of the number of c-Fos-immunoreactive cells}

There was a significant decrease in the amount of c-Fos-ir cells in the dentate nucleus $\left(H_{2}=6.343, p<0.05\right.$; Figure 4.2 and 4.3$)$ and in the fastigial nucleus $\left(H_{2}=6.870, p<0.05\right.$; Figure 4.2 and 4.3) after DBS. As expected, we found a lower amount of c-Fos-ir cells in the dentate and fastigial nucleus of the stimulated rats compared to the control rats. Post hoc analysis showed that in the rats stimulated in the dIPAG, the difference with control rats was significant for both nuclei. (DN: $U=1.00, r=-2.21 ; F N$ : $U=0.00, r=-2.45$ ). When comparing $\mathrm{VMH}$-stimulated rats to control rats this difference was significant only in the nucleus dentatus $(U=1.00, r=-2.02)$; the difference in the fastigial nucleus showed a trend towards significance $(U=2.00, r=-1.73)$. There was no significant difference in the amount of c-fos-ir cells between the stimulated groups ( $D N: U=7.00$, $r=-0.74 ; F N$ : $U=6.00, r=-0.98$ ).

Analysis of the interpositus nucleus (IN) showed a similar trend towards decreased c-Fos expression in the stimulated rats compared to controls (PAG: U $=19.00$, $r=-1.04 ; V M H: U=21.00, r=-1.37)$. Again there was no difference between the stimulated groups $(U=36.00, r=-0.36)$. 

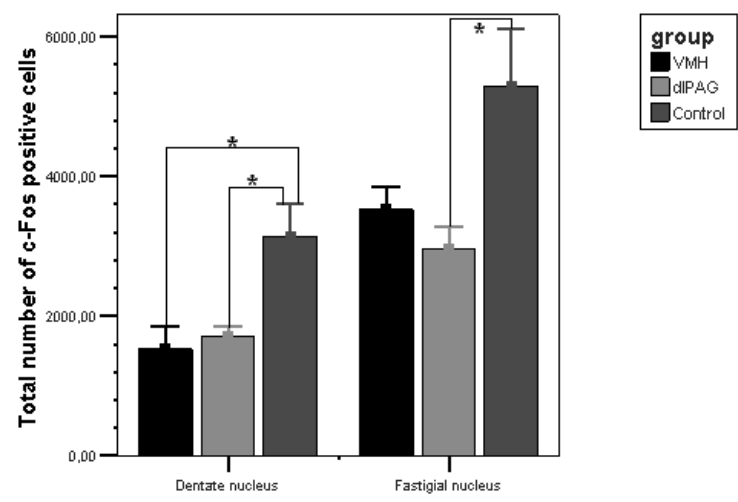

Figure 4.2 C-Fos expression in the dentate nucleus and in the fastigial nucleus of the cerebellum. Data are represented as means plus S.E.M. showing control animals, animals stimulated in the dorsolateral periaqueductal grey (dIPAG) and animals stimulated in the ventromedial hypothalamus (VMH). The asterisk $\left({ }^{*}\right)$ indicates a significant difference between groups. Note the significantly lower numbers of c-Fos immunoreactive neurons in the groups with animals showing panic attacks evoked by stimulation of the dIPAG and VMH.

\section{Discussion}

The objective of our experiment was to use electrical stimulation of the dorsolateral periaqueductal grey and of the ventromedial hypothalamus in rats as a model of panic attack to study the involvement of the deep cerebellar nuclei. We have found that panic-like behaviour was accompanied by a decrease of c-Fos-ir cells in the DCbN, indicating deactivation. c-Fos expression was significantly lower in the DN of both treatment groups and in the FN of the dIPAG DBS group when compared to controls. In the VMH DBS group, c-Fos expression in the FN was lower compared to controls, with a trend towards significance. Using a semiquantitative analysis, the IN showed a similar trend towards decreased c-Fos expression in the stimulated rats compared to controls. This should be considered a preliminary finding since the method of counting was not performed using stereological principles.

There is anatomical and functional evidence supporting the role of the cerebellum in panic. Sakai and colleagues found a significantly higher glucose uptake in the cerebellum of patients with panic disorder compared to control patients ${ }^{20}$. After clinical improvement of these patients due to cognitive-behavioural therapy, the glucose uptake in cerebellum had decreased ${ }^{21}$. Several groups found increased activation of the cerebellum after CCK-4-induced panic attacks in healthy subjects ${ }^{24,25}$. This increase was not seen in these subjects during anticipatory anxiety. 


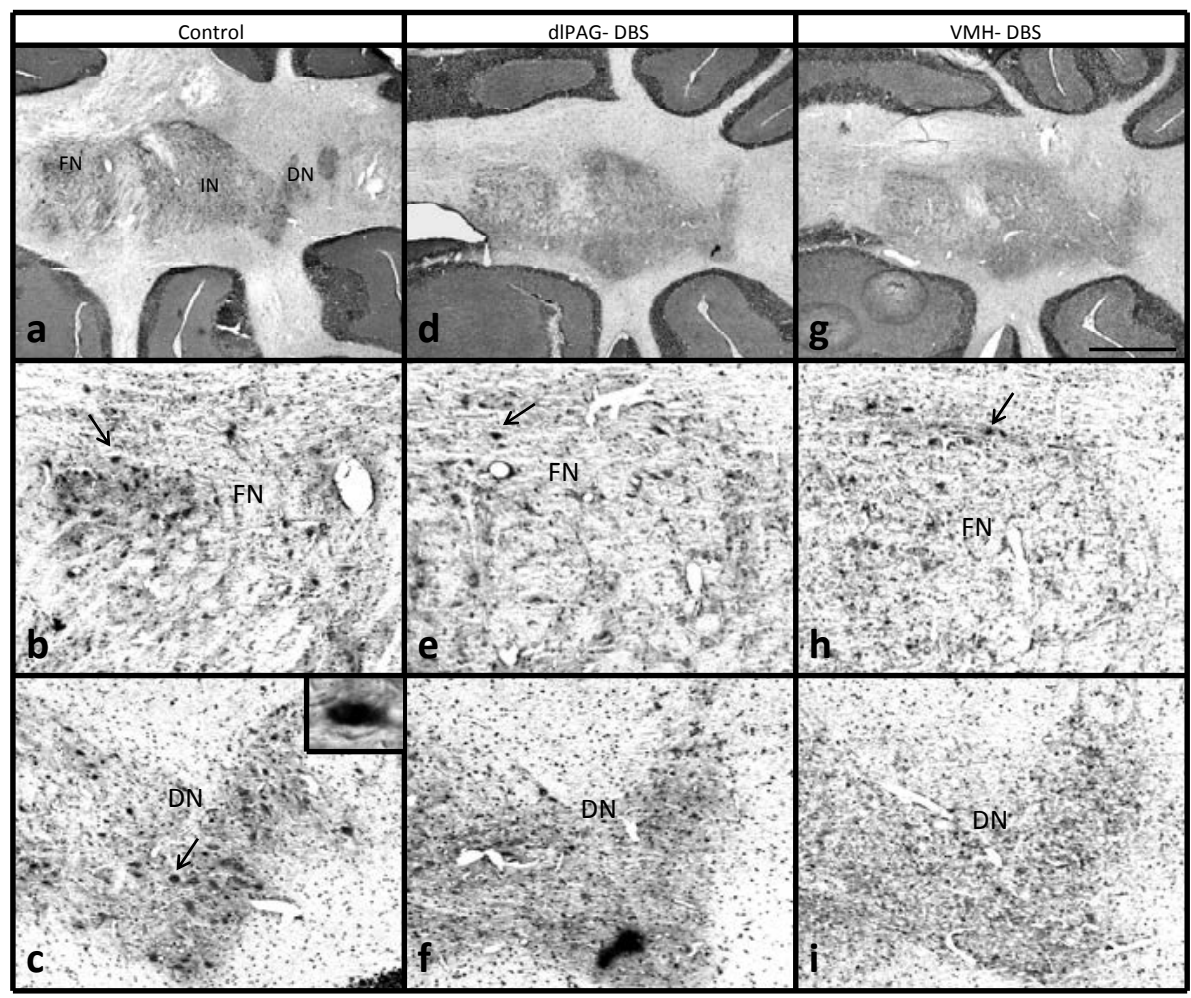

Figure 4.3 Representative low-power photomicrographs of c-Fos expression in a $10 \mu \mathrm{m}$-thick horizontal section of the deep cerebellar nuclei at bregma level $-6.10 \mathrm{~mm}$ (according to the stereotactic rat brain atlas of Paxinos and Watson of 1998) in a sham rat (a, b, c), a rat stimulated in the dIPAG (d, e f), and a rat stimulated in the VMH (g, h, i). Figures $a, d$, and g were taken at $2 \mathrm{X}$ magnification; and figures b, c, e, f, h, and i were taken at high-power magnification. The arrows point to a c-Fos-ir cell, represented by a small dark dot. The inset in c shows a representative high-power photomicrograph of a c-Fos-ir cell. Scale bar for $2 \mathrm{X}$ magnification power=1 mm. dIPAG: dorsolateral periaqueductal grey; VMH: ventromedial hypothalamus; DBS: deep brain stimulation; DN: dentate nucleus of the cerebellum; FN: fastigial nucleus of the cerebellum.

These studies were not initially designed to analyse changes in the cerebellum, and the changes found are usually unexpected. The findings are often simply mentioned as interesting or surprising, although several groups relate to the role of the cerebellum in fear conditioning as proposed by Sacchetti and others ${ }^{38}$. Sacchetti and coworkers reviewed the evidence showing that the cerebellar vermis plays a role in the fear response and in fear conditioning, especially in fear consolidation. The most important changes are thought to take place at the level of the Purkinje cell (PC). It has been shown that several forms of fear conditioning lead to increased PC excitability and an increased firing rate of the PCs. In contrast, heterozygous Lurcher mice, which show early and complete apoptosis of cerebellar PCs, show reduced inhibition to 
anxiety-provoking aversive areas ${ }^{39}$. In summary, it seems that increased PC activity leads to more fear and decreased or absent PC activity to less fear. Functional imaging is designed to analyse activation changes in the cerebral cortex; therefore, it is likely that the activation seen in the cerebellum is also located in the cortex. Increased activation found in functional imaging studies may therefore reflect the increased activity of the PCs. Since PCs are known to have an inhibitory action on the DCbN, these findings are in line with deactivation that we found in the DCbN in the stimulated rats which show panic-like behaviour.

In the cerebellum, the vermis, projecting through the fastigial nucleus, seems to be the most important structure in fear and panic. In the cases described by Schmahmann and colleagues the vermis was always involved in patients with changes of affect ${ }^{5}$. In early experiments vermal lesions were shown to attenuate a variety of fear behaviour, whereas vermal stimulation leads to increased fear-related responses ${ }^{40,41}$ Other animal research shows a selective role for the fastigial nucleus in heart rate conditioning ${ }^{40,42,43}$. The vermis also contributes to consolidation of fear memory ${ }^{44}$. There is also evidence of a role of the interpositus nucleus and the dentate nucleus in fear. In studies investigating several aspects of fear conditioning, animals with lesions of the dentate and interpositus nucleus do not acquire an aversive conditioned response, but they do acquire an appetitive conditioned response ${ }^{45}$ and show unaltered heart-rate conditioning ${ }^{42}$ and vocalisation indicative of unspecific fear $^{46}$. Furthermore, there are clear bi-directional connections to the hypothalamus from (greatest to least concentration) the dentate nucleus, the interpositus nucleus and the fastigial nucleus ${ }^{47-50}$, supporting a role of all DCbN in autonomic processes, for example those related to fear. In addition, the $\mathrm{DCbN}$ have been shown to project to several parts of the fear network: Teune and colleagues injected tracers in the DCbN in rats and documented projections to the PAG from the fastigial nucleus and the dentate nucleus and, in lesser degree, from the interpositus nucleus ${ }^{51}$. Several groups have suggested projections from the fastigial nucleus to the amygdala, hippocampus, septal nuclei and the nucleus accumbens, based on behavioural changes following cerebellar stimulation or lesioning ${ }^{38,41,52}$. Berntson and Torello, for example, showed that hyperemotionality caused by septal lesions was largely attenuated by lesions of the fastigial nucleus ${ }^{53}$. An exact anatomical pathway has never been shown ${ }^{54}$. However, these behavioural changes indicate that the involvement of the cerebellum in fear does not merely consist of regulation of an autonomic visceromotor response but that there is a place for the cerebellum in the network regulating fear processing ${ }^{38}$. In a recent review, Stoodley and Schmahmann present a functional somatotopy of the non-motor functions of the cerebellum based on functional imaging ${ }^{55}$. They conclude that the vermis of the posterior lobe seems to be specifically related to emotional processing, whereas activation in the posterior cerebellar hemispheres may be related to the decision-making aspects of the tasks used in the experimental setting ${ }^{55}$. On the other hand, Timman and colleagues reviewed anatomical evidence for a role of the cerebellum in emotional and cognitive learning, and they conclude that with respect to 
fear the vermis, projecting through the fastigial nucleus, contributes to the autonomic and somatic aspects, whereas the posterolateral cerebellar hemispheres, projecting through the dentate and interpositus nucleus, play a role in the emotional content of fear processing ${ }^{40}$.

In summary there is ample evidence that all cerebellar nuclei are involved in fear, in which the fastigial nucleus possibly mediates a different aspect of fear than the dentate nucleus and the interpositus nucleus. This correlates with our findings in the present study showing a similar deactivation in the dentate nucleus and in the fastigial nucleus, and possibly also in the interpositus nucleus. Increased fear is associated with increased PC activity in functional imaging studies, which is hypothesised to lead to inhibition of the $\mathrm{DCbN}$ and therefore of cerebellar output. We speculate that the cerebellum plays a role in regulating appropriate behaviour in response to any stimulus, and that a decreased cerebellar output may play a role in emergence of an inappropriate response, such as a panic attack. This decreased output may be in response to incorrect input, as suggested by Parvizi and colleagues ${ }^{31}$; however, we suggest that the cerebellum also plays a direct role in the selection of relevant information on which an adequate behavioural response is based, and that deactivation of the $\mathrm{DCbN}$ then leads to inappropriate behaviour by inhibiting this selection process.

\section{Conclusion}

This study supports the hypothesis that the cerebellum is involved in panic attacks. Using DBS of the dIPAG and the VMH in rats as a model of a panic attack, we found that the activity of the DCbN as measured by c-Fos expression decreased significantly in the dentate nucleus and in the fastigial nucleus, and possibly also in the interpositus nucleus. In a previous study, we showed that the $\mathrm{DCbN}$ are de-activated in rats showing increased impulsivity. In conclusion, deactivation of the cerebellum is associated with inappropriate behaviour such as panic and impulsivity. We suggest that the cerebellum plays a role in the selection of relevant information, thereby inhibiting such inappropriate behaviour. 


\section{References}

1. Fine EJ, lonita CC, Lohr L. The history of the development of the cerebellar examination. Semin Neurol. 2002;22:375-84.

2. Schmahmann JD. Disorders of the cerebellum: ataxia, dysmetria of thought, and the cerebellar cognitive affective syndrome. J Neuropsychiatry Clin Neurosci. 2004;16:367-78.

3. Leiner HC, Leiner AL, Dow RS. Does the cerebellum contribute to mental skills? Behav Neurosci. 1986;100:443-54.

4. Moers-Hornikx VM, Sesia T, Basar K, Lim LW, Hoogland G, Steinbusch HW, Gavilanes DA, Temel Y, Vles JS. Cerebellar nuclei are involved in impulsive behaviour. Behav Brain Res. 2009;203:256-63.

5. Schmahmann JD, Sherman JC. The cerebellar cognitive affective syndrome. Brain. 1998;121:561-79.

6. Baldacara L, Borgio JG, de Lacerda AL, Jackowski AP. Cerebellum and psychiatric disorders. Rev Bras Psiquiatr. 2008;30:281-9.

7. Hoppenbrouwers SS, Schutter DJ, Fitzgerald PB, Chen R, Daskalakis ZJ. The role of the cerebellum in the pathophysiology and treatment of neuropsychiatric disorders: a review. Brain Res Rev. 2008;59: 185-200.

8. Andreasen NC, Pierson R. The role of the cerebellum in schizophrenia. Biol Psychiatry. 2008;64:81-8.

9. Picard H, Amado I, Mouchet-Mages S, Olie JP, Krebs MO. The role of the cerebellum in schizophrenia: an update of clinical, cognitive, and functional evidences. Schizophr Bull. 2008; 34:155-72.

10. Palmen SJ, van Engeland H, Hof PR, Schmitz C. Neuropathological findings in autism. Brain. 2004;127(Pt 12):2572-83.

11. Pierce $\mathrm{K}$, Courchesne E. Evidence for a cerebellar role in reduced exploration and stereotyped behavior in autism. Biol Psychiatry. 2001;49:655-64.

12. Levinson HN. The cerebellar-vestibular predisposition to anxiety disorders. Percept Mot Skills. 1989;68:323-38.

13. De Bellis MD, Kuchibhatla M. Cerebellar volumes in pediatric maltreatment-related posttraumatic stress disorder. Biol Psychiatry. 2006;60:697-703.

14. Busatto GF, Zamignani DR, Buchpiguel CA, Garrido GE, Glabus MF, Rocha ET, Maia AF, Rosario-Campos MC, Campi Castro C, Furuie SS, Gutierrez MA, McGuire PK, Miguel EC. A voxel-based investigation of regional cerebral blood flow abnormalities in obsessive-compulsive disorder using single photon emission computed tomography (SPECT). Psychiatry Res. 2000;99:15-27.

15. Kang DH, Kwon JS, Kim JJ, Youn T, Park HJ, Kim MS, Lee DS, Lee MC. Brain glucose metabolic changes associated with neuropsychological improvements after 4 months of treatment in patients with obsessive-compulsive disorder. Acta Psychiatr Scand. 2003;107:291-7.

16. Nabeyama M, Nakagawa A, Yoshiura T, Nakao T, Nakatani E, Togao O, Yoshizato C, Yoshioka K, Tomita $\mathrm{M}$, Kanba S. Functional MRI study of brain activation alterations in patients with obsessive-compulsive disorder after symptom improvement. Psychiatry Res. 2008;163:236-47.

17. Kilts CD, Kelsey JE, Knight B, Ely TD, Bowman FD, Gross RE, Selvig A, Gordon A, Newport DJ, Nemeroff $\mathrm{CB}$. The neural correlates of social anxiety disorder and response to pharmacotherapy. Neuropsychopharmacology. 2006;31:2243-53.

18. Tillfors M, Furmark T, Marteinsdottir I, Fredrikson M. Cerebral blood flow during anticipation of public speaking in social phobia: a PET study. Biol Psychiatry. 2002;52:1113-9.

19. Warwick JM, Carey P, Jordaan GP, Dupont $P$, Stein DJ. Resting brain perfusion in social anxiety disorder: a voxel-wise whole brain comparison with healthy control subjects. Prog Neuropsychopharmacol Biol Psychiatry. 2008;32:1251-6.

20. Sakai Y, Kumano H, Nishikawa M, Sakano Y, Kaiya H, Imabayashi E, Ohnishi T, Matsuda H, Yasuda A, Sato A, Diksic M, Kuboki T. Cerebral glucose metabolism associated with a fear network in panic disorder. Neuroreport. 2005;16:927-31.

21. Sakai Y, Kumano H, Nishikawa M, Sakano Y, Kaiya H, Imabayashi E, Ohnishi T, Matsuda H, Yasuda A, Sato A, Diksic M, Kuboki T. Changes in cerebral glucose utilization in patients with panic disorder treated with cognitive-behavioral therapy. Neuroimage. 2006;33:218-26.

22. Pujol J, Soriano-Mas C, Alonso P, Cardoner N, Menchón JM, Deus J, Vallejo J. Mapping structural brain alterations in obsessive-compulsive disorder. Arch Gen Psychiatry. 2004;61:720-30. 
23. Javanmard M, Shlik J, Kennedy SH, Vaccarino FJ, Houle S, Bradwejn J. Neuroanatomic correlates of CCK-4-induced panic attacks in healthy humans: a comparison of two time points. Biol Psychiatry. 1999;45:872-82.

24. Eser D, Leicht G, Lutz J, Wenninger S, Kirsch V, Schüle C, Karch S, Baghai T, Pogarell O, Born C, Rupprecht R, Mulert C. Functional neuroanatomy of CCK-4-induced panic attacks in healthy volunteers. Hum Brain Mapp. 2009;30:511-22.

25. Schunck T, Erb G, Mathis A, Gilles C, Namer IJ, Hode Y, Demaziere A, Luthringer R, Macher JP. Functional magnetic resonance imaging characterization of CCK-4-induced panic attack and subsequent anticipatory anxiety. Neuroimage. 2006;31: 1197-208.

26. Benkelfat C, Bradwejn J, Meyer E, Ellenbogen M, Milot S, Gjedde A, Evans A. Functional neuroanatomy of CCK4-induced anxiety in normal healthy volunteers. Am J Psychiatry. 1995;152:1180-4.

27. Roy-Byrne PP, Craske MG, Stein MB. Panic disorder. Lancet. 2006;368:1023-32.

28. Gorman JM, Kent JM, Sullivan GM, Coplan JD. Neuroanatomical hypothesis of panic disorder, revised. Am J Psychiatry. 2000;157:493-505.

29. Lim LW, Blokland A, Visser-Vandewalle V, Vlamings R, Sesia T, Steinbusch H, Schruers K, Griez E, Temel Y. High-frequency stimulation of the dorsolateral periaqueductal gray and ventromedial hypothalamus fails to inhibit panic-like behaviour. Behav Brain Res. 2008;193:197-203.

30. Schenberg LC, Bittencourt AS, Sudre EC, Vargas LC. Modeling panic attacks. Neurosci Biobehav Rev. 2001;25:647-59.

31. Parvizi J, Anderson SW, Martin CO, Damasio H, Damasio AR. Pathological laughter and crying: a link to the cerebellum. Brain. 2001;124(Pt 9):1708-19.

32. Sharp FR, Sagar SM, Hicks K, Lowenstein D, Hisanaga K. c-fos mRNA, Fos, and Fos-related antigen induction by hypertonic saline and stress. J Neurosci. 1991;11:2321-31.

33. Smith MA, Banerjee S, Gold PW, Glowa J. Induction of c-fos mRNA in rat brain by conditioned and unconditioned stressors. Brain Res. 1992;578:135-41.

34. Temel Y, Boothman L, Blokland A, Magill PJ, Steinbusch HW, Visser-Vandewalle V, Sharp T. Inhibition of 5-HT neuron activity and induction of depressive-like behavior by high-frequency stimulation of the subthalamic nucleus. Proc Natl Acad Sci U S A. 2007;104:17087-92.

35. Temel Y, Visser-Vandewalle V, van der Wolf M, Spincemaille GH, Desbonnet L, Hoogland G, Steinbusch HW. Monopolar versus bipolar high frequency stimulation in the rat subthalamic nucleus: differences in histological damage. Neurosci Lett. 2004;367:92-6.

36. Abercrombie M. Estimation of nuclear population from microtome sections. Anatomical Record. 1946;94:239-47.

37. Tunc AT, Turgut M, Aslan H, Sahin B, Yurtseven ME, Kaplan S. Neonatal pinealectomy induces Purkinje cell loss in the cerebellum of the chick: a stereological study. Brain Res. 2006; 1067:95-102.

38. Sacchetti B, Scelfo B, Strata P. The cerebellum: synaptic changes and fear conditioning. Neuroscientist. 2005;11:217-27.

39. Lorivel T, Hilber P. Effects of chlordiazepoxide on the emotional reactivity and motor capacities in the cerebellar Lurcher mutant mice. Behav Brain Res. 2006;173:122-8.

40. Timmann D, Drepper J, Frings M, Maschke M, Richter S, Gerwig M, Kolb FP. The human cerebellum contributes to motor, emotional and cognitive associative learning. A review. Cortex. 2010;46:845-57.

41. Snider RS, Maiti A. Cerebellar contributions to the Papez circuit. J Neurosci Res. 1976;2: 133-46.

42. Lavond DG, Lincoln JS, McCormick DA, Thompson RF. Effect of bilateral lesions of the dentate and interpositus cerebellar nuclei on conditioning of heart-rate and nictitating membrane/eyelid responses in the rabbit. Brain Res. 1984;305:323-30.

43. Supple WF, Jr., Leaton RN. Lesions of the cerebellar vermis and cerebellar hemispheres: effects on heart rate conditioning in rats. Behav Neurosci. 1990;104:934-47.

44. Sacchetti B, Scelfo B, Tempia F, Strata P. Long-term synaptic changes induced in the cerebellar cortex by fear conditioning. Neuron. 2004;42:973-82.

45. Steinmetz JE, Logue SF, Miller DP. Using signaled barpressing tasks to study the neural substrates of appetitive and aversive learning in rats: behavioral manipulations and cerebellar lesions. Behav Neurosci. 1993;107:941-54.

46. Lee T, Kim JJ. Differential effects of cerebellar, amygdalar, and hippocampal lesions on classical eyeblink conditioning in rats. J Neurosci. 2004;24:3242-50. 
47. Haines DE, Dietrichs E, Mihailoff GA, McDonald EF. The cerebellar-hypothalamic axis: basic circuits and clinical observations. Int Rev Neurobiol. 1997;41:83-107.

48. Zhu JN, Zhang YP, Song YN, Wang JJ. Cerebellar interpositus nuclear and gastric vagal afferent inputs reach and converge onto glycemia-sensitive neurons of the ventromedial hypothalamic nucleus in rats. Neurosci Res. 2004;48:405-17.

49. Çavdar S, San T, Aker R, Sehirli ÜS, Onat FYI. Cerebellar connections to the dorsomedial and posterior nuclei of the hypothalamus in the rat. J Anat. 2001;198:37-45.

50. Dietrichs E, Haines DE. Possible pathways for cerebellar modulation of autonomic responses: micturition. Scand J Urol Nephrol Suppl. 2002(210):16-20.

51. Teune TM, Burg Jvd, Moer Jvd, Voogd J, Ruigrok TJH. Topography of cerebellar nuclear projections to the brain stem in the rat. Prog Brain Res. 2000;124:141-72.

52. Turner BM, Paradiso S, Marvel CL, Pierson R, Boles Ponto LL, Hichwa RD, Robinson RG. The cerebellum and emotional experience. Neuropsychologia. 2007;45:1331-41.

53. Berntson GG, Torello MW. Attenuation of septal hyperemotionality by cerebellar fastigial lesions in the rat. Physiol Behav. 1980;24:547-51.

54. Strick PL, Dum RP, Fiez JA. Cerebellum and nonmotor function. Annu Rev Neurosci. 2009;32:413-34.

55. Stoodley CJ, Schmahmann JD. Functional topography in the human cerebellum: a meta-analysis of neuroimaging studies. Neuroimage. 2009;44:489-501. 



\section{Chapter 5}

c-Fos expression in the deep cerebellar nuclei in a rat model of conditioned fear

VMP Moers-Hornikx, JSH Vles, RJ Hemmes, LW Lim, G Hoogland, HWM Steinbusch, Y Temel

J Exp Clin Med. 2012;29: 201-7 


\section{Abstract}

In contrast to what was previously believed, there is increasing evidence that the cerebellum is involved in emotional and cognitive behaviour. Preclinical and clinical research points towards a role of the cerebellum in impulsivity and panic. Deep brain stimulation (DBS) of the dorsolateral periaqueductal gray (dIPAG) in rats has proved to elicit escape behaviour directly, but can also be used for conditioning of context-dependant fear responses. We used this model to study the activation in the deep cerebellar nuclei $(D C b N)$ after conditioned fear. For this purpose, we performed c-Fos immunohistochemistry in the DCbN.

We found no statistically significant difference in c-Fos expression in the DCbN between the subgroups in this animal model. We hypothesize that the lack of a significant difference in activation of the DCbN may be related to fear consolidation which takes place after dIPAG stimulation, after which retrieval of behaviour, whether adequate or inadequate, does not lead to a different activation of the $\mathrm{DCbN}$. On the other hand the effect of conditioned fear may be smaller than the effect of panic behaviour elicited by DBS, requiring a larger study population. 


\section{Introduction}

The cerebellum has been regarded for decades to be a neuroanatomical structure solely involved in the control of motor coordination ${ }^{1-4}$. However, there is now more than enough evidence that the cerebellum also plays a prominent role in cognitive and behavioural processes ${ }^{5-7}$. The evolutionary newer regions of the cerebellum project to the prefrontal cortex (areas 9 and 46) and the inferior parietal lobe area 7 through the ventrolateral and other thalamic nuclei including the mediodorsal thalamic nucleus and the reticular nucleus ${ }^{8-13}$. The cerebellum also receives a wide range of input from structures involved in cognition and affect ${ }^{14-21}$. These structures form the so called cerebrocerebellar circuit for cognition and emotion ${ }^{9,22}$. Behavioural disturbances resulting from isolated damage to the cerebellum comprise a constellation of symptoms which has been called the cerebellar cognitive affective syndrome $(\text { CCAS })^{23-25}$. These include impairment in executive functions, difficulties with spatial cognition, memory deficiencies, personality changes with disinhibited and inappropriate behaviour, language deficits, and mutism ${ }^{7,26-30}$.

In our previous research we have shown that, in a rat model, impulsivity induced by deep brain stimulation (DBS) of the mediodorsal thalamic nucleus (MD) is associated with deactivation of the deep cerebellar nuclei $(D C b N)^{31}$. In rats in which DBS of the dorsolateral periaqueductal grey (dIPAG) induces panic-like behaviour we have shown the same association ${ }^{32}$. In contrast rats stimulated in the subthalamic nucleus (STN), known to result in a decrease in impulsivity, show increased activation in the $\mathrm{DCbN}^{33,34}$. We hypothesize that the cerebellum plays a role in the selection of relevant information on which a behavioural response is based, thereby coordinating an appropriate response to a stimulus. Deactivation of the cerebellar nuclei may then lead to inappropriate behaviour.

Aside from a role in acute panic, it has been shown that the cerebellum plays a significant role in fear consolidation and fear memory, and therefore also in conditioned fear ${ }^{35,36}$. Lim et al. showed that the dIPAG stimulated rat is not only a good model for acute induced fear- and panic like behaviour, but can also be used for conditioning context dependent fear responses ${ }^{37}$. In the same study this panic behaviour was also shown to be moderated on a behavioural level by escitalopram, with other research stating that similar antidepressants can reduce fos immunoreactivity in the periaqueductal grey $^{37,38}$. In the present study we performed DBS of the dIPAG in rats to induce conditioned fear. These rats were treated chronically with escitalopram or buspiron. We then determined the activation of the DCbN as measured by the amount of c-Fos immunoreactivity two hours after a conditioned fear response. As we have previously shown that panic behaviour induced by dIPAG stimulation is accompanied by a decrease of c-Fos expression in the DCbN, we now expected to find a decreased c-Fos expression in the animals showing a conditioned fear response compared to the animals that don't show this conditioned fear response $^{32,37}$. 


\section{Materials and methods}

\section{Subjects}

Male albino Wistar rats ( $n=40,12$ weeks old, bred and housed at the Central Animal Facility of Maastricht University, the Netherlands). Animals were housed individually in standard cages on sawdust bedding in an air ventilated room (temperature approximately $20^{\circ} \mathrm{C}$ ) under a $12 / 12$-h reversed light/ dark cycle. Standard laboratory chow (Hopefarms, Woerden, the Netherlands) and water were available ad libitum. At the time of surgery the average body weight of the rats was 300-350 g. The present study was approved by the Animal Experiments and Ethics Committee of Maastricht University.

\section{Experimental groups}

Rats were randomly assigned to one of the following six experimental groups: A. dIPAG stimulation and treatment with saline $(n=7) ; B$. dIPAG stimulation and treatment with buspirone $(n=7)$; C. dIPAG stimulation and treatment with escitalopram $(n=7)$; D. dIPAG sham surgery and saline treatment $(n=6)$; E. dIPAG sham surgery and buspirone treatment $(n=6)$; and F. dIPAG sham surgery and escitalopram treatment $(n=7)$.

\section{Surgical procedures}

A description of the surgical procedure has been previously reported ${ }^{39-41}$. Rats were anesthetized using ketamine $(90 \mathrm{mg} / \mathrm{kg})$ and xylazine $(10 \mathrm{mg} / \mathrm{kg})$ injected subcutaneously (s.c.). By means of a stereotactic apparatus (Stoelting, Wood Dale, USA; model 51653) burr holes were made in the skull through which a gold-plated electrode with inner platinum- iridium combination was implanted (Technomed, Beek, the Netherlands) at the level of the right dIPAG (coordinates from Bregma: anteroposterior, $-7.6 \mathrm{~mm}$; mediolateral, $+0.7 \mathrm{~mm}$; and ventral, $-4.8 \mathrm{~mm}$; approached with a coronal angle of $10^{\circ}$; Paxinos and Watson, 1998). The electrodes were fixed in position using dental cement (Heraeus Kulzer, Hanau, Germany). After surgery the rats were injected with Temgesic $(0.1 \mathrm{mg} / \mathrm{kg}$, s.c.) for analgesia and allowed to recover for a period of two weeks.

\section{Deep brain stimulation}

Stimulation was performed at a frequency of $50 \mathrm{~Hz}$ and pulse width of $0.1 \mathrm{~ms}$ based on previous experience ${ }^{37}$. The amplitude was gradually increased until escape behaviour was observed. Stimulation at each amplitude was performed during $15 \mathrm{~s}$ followed by a stimulation-off period of $45 \mathrm{~s}$. Two consecutive positive responses of escape reaction were required to determine the escape threshold. The mean amplitude neccesary to elicit an escape response was $60 \mu \mathrm{A}$. Animals which required stimulation intensities above $100 \mu \mathrm{A}$, suggesting incorrect positioning of the electrode, were discarded from 
analysis. A World Precision Instruments (WPI) digital stimulator (DS8000, WPI, Berlin, Germany) and a stimulus isolator (DLS100, WPI, Berlin, Germany) were used to deliver the stimuli. After the threshold determination session, all rats were given a recovery period of two weeks. The sham animals were similarly connected to the stimulator but no current was delivered to the dIPAG.

\section{Drug administration}

Escitalopram oxalate ( $H$. Lundbeck A/S, Copenhagen, Denmark) and buspirone hydrochloride (TOCRIS, Cookson Inc., Missouri,USA) were dissolved in saline $0.9 \% \mathrm{NaCl}$, as described previously ${ }^{37}$. Habituation to the injection procedure was obtained by injection of $1 \mathrm{ml}$ saline three times on alternating days one week before the actual experimental testing. The animals received 21 daily injections of either escitalopram (ESCIT, s.C., $10 \mathrm{mg} / \mathrm{ml}, 1 \mathrm{ml} / \mathrm{kg}$ ), buspiron (BUSP, s.C., $3 \mathrm{mg} / \mathrm{ml}, 1 \mathrm{ml} / \mathrm{kg}$ ), or saline (SAL, $1 \mathrm{ml} / \mathrm{kg}$ ), respectively. Chronic treatment was chosen as the maximal effect of these drugs is expected in the chronic phase ${ }^{37,42-46}$. The final drug administration was performed $60 \mathrm{~min}$ (ESCIT, SAL) or $120 \mathrm{~min}$ (BUSP) before the test in the open-field.

\section{Behavioural testing}

Rats were tested in an open field (OF). Details of this test were published previously by Lim et al. ${ }^{37}$. The OF consisted of a clear Plexiglas box (square: $100 \mathrm{~cm} \times 100 \mathrm{~cm}$, and height: $40 \mathrm{~cm}$ ) with open top and dark floor ${ }^{46}$. All open-field testing was conducted in the same room in a dimly lit condition. On the day of stimulation in the open-field, rats were placed in the open-field arena and connected with the external stimulator through externalized leads followed after 1 minute by electrical stimulation with parameters based on the previously determined escape threshold. Once the escape reaction was evoked, the cable connected to the rat was detached and the animal was left in the open-field for approximately $30 \mathrm{~s}$. The electrical stimulation to elicit an escape reaction was always carried out between 20:00 and 22:00 $\mathrm{h}$.

Twelve hours after the induced escape behaviour (between 08:00 and 10:00 h) animals were placed in the OF again during which the behaviour of each rat was recorded using an automated system consisting of a camera connected to a computer with the Ethovision tracking software (Ethovision, Noldus Information Technology, Wageningen, the Netherlands). The Ethovision software automatically calculated and analysed temporospatial data of the animal relative to the OF including locomotion / distance moved, velocity and time spent in the corner of the open field, each parameter on a per 2 minutes basis. A trial was stopped after 5 minutes and the rat was removed from the arena. Fear or freezing behaviour was defined by a decreased locomotion (immobility) and increased duration of time spent in the corner of the OF. Escape behaviour was characterized by rigorous and aimless running within the OF arena. 


\section{Histological processing}

Two hours after the final testing of the rats in the OF they were placed under generalized anaesthesia with Nembutal $(75 \mathrm{mg} / \mathrm{kg})$ and transcardially perfused with Tyrode $(0.1 \mathrm{M})$ and a fixative solution which consists of paraformaldehyde, picric acid and glutaraldehyde in phosphate buffer $(\mathrm{pH}$ 7.6). After two hours of post fixation the brains were cryoprotected by overnight sucrose and then quickly frozen with $\mathrm{CO}_{2}$ and stored at $-80^{\circ} \mathrm{C}$. One series of brainstem sections per animal was stained with standard hematoxylin-eosin (Merck, Darmstadt, Germany) to examine the localization of the electrode tips.

The cerebelli were cut serially on a cryostat (MICROM, Walldorf, Germany) into $10 \mu \mathrm{m}$ frontal sections which were collected on gelatin-coated slides and then stored at $-30^{\circ} \mathrm{C}$. We used a previously published protocol for c-Fos immunohistochemistry with minor modifications ${ }^{32}$. In short, we used a primary rabbit polyclonal IgG antibody against c-Fos (diluted 1:300, Santa Cruz Biotechnology Inc., Santa Cruz, CA, USA) for incubation at room temperature for two days on a constant shaker. Subsequently sections were incubated with the secondary antibody (diluted 1:400, biotinylated donkey anti- rabbit biotin; Jackson Immunoresearch laboratories Inc., Westgrove, USA) overnight. This was followed by incubation for 2 hours with an avidin-biotin-peroxidase complex (diluted 1:800, Elite ABC-kit, Vectastatin; Vector, Burlingame, USA). To visualize the immune complex of horseradish peroxide (HRP) reaction product, the sections were incubated with 3,3'-diaminobenzidine tetrahydrochloride (DAB)/nickel chloride $\left(\mathrm{NiCl}_{2}\right)$ solution. Finally sections were dehydrated and cover slipped with Pertex (HistolabProducts, Goteborg, Sweden).

A Nissl stain was conducted on a parallel series of $10 \mu \mathrm{m}$ sections to facilitate delineation of the boundaries of the $\mathrm{DCbN}$. This parallel series was first air-dried for 30 minutes and subsequently exposed to a buffer solution $(40 \mathrm{ml} 136.08 \mathrm{~g} / \mathrm{mol}$ sodium acetate with $9.6 \mathrm{ml}$ glacial acetic acid in a $1000 \mathrm{ml}$ distilled water solution (milli-q)), followed by a $2 \%$ triton $x-100$ solution of $2.5 \mathrm{ml}$ with $150 \mathrm{ml}$ absolute alcohol and $50 \mathrm{ml}$ distilled water, then the previously described buffer solution again, and finally, a Cresyl violet solution $(0.1 \mathrm{~g} / 1000 \mathrm{ml}$ distilled water). Finally sections were rinsed with the buffer solution, dehydrated, and cover slipped with Pertex (HiStolabProducts, Goteborg, Sweden).

\section{Quantative evaluation of c-Fos immunoreactive cells}

Systematic cell counts of c-Fos immunoreactive (c-Fos-ir) cells were performed in the DCbN as previously described ${ }^{34}$. Photography of both the c-Fos and parallel Nissl stained sections was performed at a $4 \mathrm{X}$ magnification on an Olympus AX70 bright-field microscope with an Olympus DP70 camera (analySIS; Imaging System, Münster, Germany). The bregma levels of the $D C b N$ ranged from -10.52 to $-11.80 \mathrm{~mm}$, which was verified with the Paxinos and Watson Atlas of $1998^{47}$. 
In the c-Fos stained sections the delineation of the DCbN was difficult due to a weak background staining. As mentioned above, a parallel series of sections was stained with a Nissl-staining to aid in delineation. Using Image J (version 1.43), a digital substraction of the $\mathrm{DCbN}$ as delineated in the Nissl stained sections were projected on the c-Fos stained slides thereby giving a more accurate delineation of the cerebellar nuclei. Minor deviations in this technique due to slight difference in bregma level were corrected for manually. Artefacts in the sections were excluded from analysis to ensure accuracy of measurements. A cell was defined as being c-Fos-immunoreactive (c-Fos-ir) if it had a $65 \%$ higher density than the corresponding background grey value of the $\mathrm{DCbN}$. The number of c-Fos-ir cells per $\mathrm{mm}^{2}$ was calculated for each nucleus.

\section{Statistical analysis}

Since we were primarily interested in the effect of conditioned fear on c-Fos expression in the $\mathrm{DCbN}$, the treatment groups were pooled according to whether they did or did not show escape behaviour in the final test in the OF. The data of the c-Fos counts were analysed using the Mann-Whitney test for non-parametric data (Monte Carlo method) since the group sizes were relatively small and non-normally distributed. The significance level was set at $p<0.05$. Furthermore we performed a Kruskall-Wallis test to analyse differences between stimulated and sham stimulated groups. Post hoc analysis to follow up significant findings from the Kruskal-Wallis test was performed using the Mann-Whitney test with a one-tailed procedure. The level of significance for the Kruskal-Wallis test was set at $p<0.05$, and for the post-hoc Mann-Whitney tests it was set at $p<0.0167$. Output is provided as the number of $c$-Fos ir cells per square millimeter with 2 standard error of the mean values (S.E.M).

Any outliers identified in the c-Fos data on a per rat per nucleus basis were corrected for by transforming the data into the maximal 2SD+/- the mean. Rats were excluded if their total average values exceeded the $2 \mathrm{SD}+/$ - the mean for the total of the groups of rats. Finally 12 rats showing freezing behaviour and 24 rats showing no freezing behaviour were included for statsitical analysis.

To determine the amount of agreement concerning the semi-quantitative method of cell counting between RJH and $\mathrm{VMH}$ an intraclass correlation coefficient (ICC) was determined on previously stained c-Fos DCbN. This was done using the Statistical Package for the Social Sciences software (SPSS, version 17.0.2, $11^{\text {th }}$ march 2009) in a two- way- mixed model of the exact type.

\section{Results}

\section{Electrode localization}

The histological evaluation of the electrode localization was previously described by Lim et al. ${ }^{37}$. The electrode tip was correctly placed in the dIPAG in six animals per group for the sham-saline, sham-buspirone, stimulation-saline and stimulation-escitalopram 
groups and seven animals for the stimulation-buspirone and sham-escitalopram $\operatorname{group}^{37}$.

\section{Behavioural results}

Results of behavioural testing were published previously by Lim et al. ${ }^{37}$. In summary electrical stimulation of the dIPAG evoked escape behaviour which was followed by decreased locomotion or a freezing response when animals were placed back in the OF 12 hours later; a behavioural pattern indicative of conditioned fear.

Escitalopram reduced the time in the corner squares of the OF in the stimulated animals but not in the sham animals $(F(2,33)=10.12, p<0.01)$. Stimulation decreased the mean velocity of movements $F(1,33)=16.57, p<0.01$ ) while escitalopram corrected for this in the stimulated animals but not the sham animals.

\section{Quantification of c-Fos-ir cells}

There were no significant differences between the left and right $\mathrm{DCbN}$, therefore the results from the left and right hemispheres were pooled for further analysis. There were no significant differences in c-Fos expression in the $D C b N$ between rats that showed conditioned fear in the final OF session and those that did not. Furthermore there were no significant differences in c-Fos expression in the DCbN between the treatment groups. (Figures 5.1-5.3).

A few cells displayed a hyperintense cytoplasma. These were not considered to be the 'classically' stained c-Fos ir cells as often described in the literature and therefore not included in the data analysis.

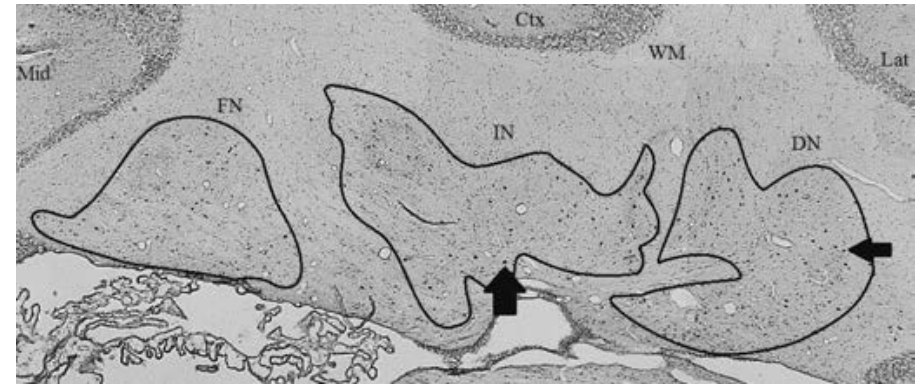

Figure 5.1 Representative low-power photomicrograph of a $10 \mu \mathrm{m}$-thick coronal section of the right cerebellar hemisphere at approximately bregma level $-11.70 \mathrm{~mm}(-11.60 \mathrm{~mm}$ to $-11.80 \mathrm{~mm})$ showing c-Fos-ir cells in the $\mathrm{DCbN}$. The $\mathrm{DCbN}$ are delineated by the black lines. The black arrows point towards a c-Fos positive cell. 4V: fourth ventricle; Ctx: cerebellar cortex; DN: dentate nucleus; FN: fastigial nucleus; IN: interposite nucleus; Lat: lateral aspect of cerebellar hemisphere; Mid: midline; Pc: choroid plexus; Wm: white matter. 

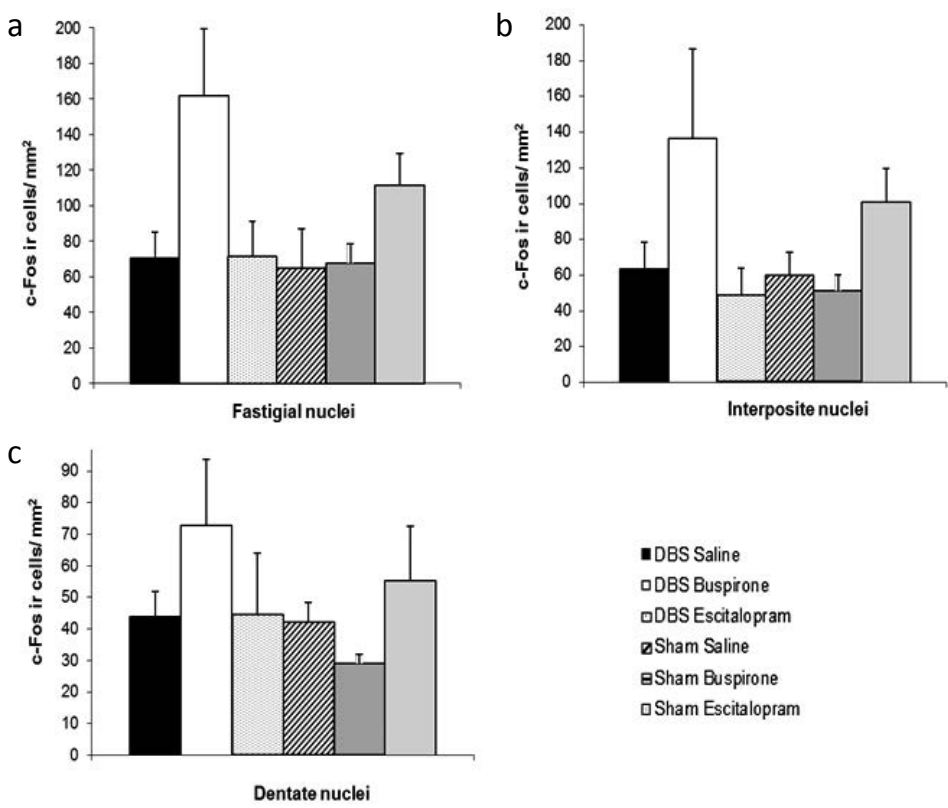

Figure 5.2 C-Fos expression in the deep cerebellar nuclei. Data are presented as mean plus S.E.M. showing six treatment groups: animals stimulated in the dorsolateral periaqueductal gray (dIPAG) and treated with respectively saline, buspiron or escitalopram, followed by animals with sham dIPAG stimulation again treated with respectively saline, buspiron or escitalopram. Data are presented for the fastigial nucleus of the cerebellum (FN; Figure 5.2a), the interposite nucleus (IN; Figure 5.2b) and the dentate nucleus (DN; Figure 5.2c). Note the higher density of c-Fos-ir cells in the dIPAG-buspiron group and in the sham-dIPAGescitalopram group which is consistently present in all nuclei, however, without reaching significance.

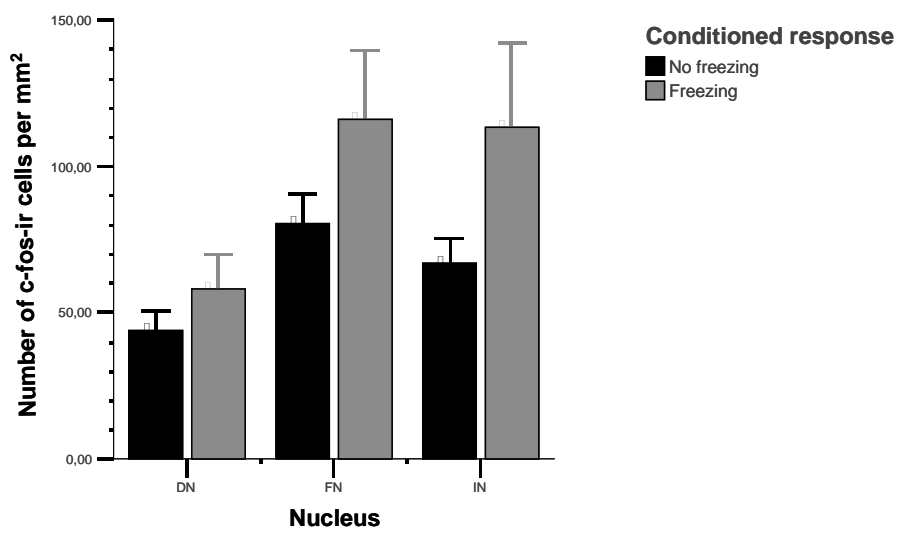

Figure 5.3 C-Fos expression in the deep cerebellar nuclei. Data are presented as mean plus S.E.M. showing the pooled treatment groups: animals showing freezing behaviour 12 hours after dorsolateral periaqueductal grey (dIPAG) stimulation and animals that don't show this freezing behaviour. Data are presented for the dentate nucleus (DN), the interposite nucleus (IN) and the fastigial nucleus (FN). 


\section{Intraclass correlation coefficient}

The intraclass correlation coefficient was determined based on the total c-Fos ir cells $/ \mathrm{mm}^{2}$ counted by $\mathrm{RJH}$ in comparison to $\mathrm{VMH}$ for all the $\mathrm{DCbN}$ in a previous experiment. The ICC for the total of the DCbN yielded a correlation of 0,795 (single value; 0.886 for average), which is a substantial to high level of agreement on the Landis \& Koch scale.

\section{Discussion}

In the present study we analysed changes in activation in the deep cerebellar nuclei after a conditioned fear response 12 hours after dorsolateral periaqueductal greystimulation. We expected a decrease in activation in the animals which show this conditioned fear response in comparison to sham stimulated animals. In the animals treated with escitalopram which do not show a conditioned fear response after dIPAG stimulation we expected a normalisation of the deactivation of the $\mathrm{DCbN}$. We were however not able to show a difference in activation in the $\mathrm{DCbN}$.

The amygdala and the hippocampal complex are important structures in fear memory ${ }^{35}$. There is also evidence for a substantial role of the cerebellum in the process of fear consolidation and retrieval. A key structure is the Purkinje cell (PC). Pairing of an unconditioned stimulus and a conditioning stimulus leads to long term depression (LTD) of the parallel fibre synapses with the PC's. This decreases the firing rate of the PC's, which allows the conditioned stimulus to be passed on to the $\mathrm{DCbN}^{35,36}$. Fear conditioning is important for survival, therefore fear memory is rapidly acquired and long lasting ${ }^{35}$. In classical Pavlovian conditioning several training sessions are usually necessary to elicit a conditioned fear response. In our study a short stimulation of the dIPAG in the open field (OF) is enough to elicit a conditioned fear response when rats are placed in the same OF arena 12 hours later ${ }^{37}$. Possibly this strong stimulus already causes synaptical changes in the cerebellum. One could then hypothesize that in the rats with a conditioned fear response, retrieval of this conditioned behaviour does not cause a different activation than retrieval of normal behaviour in the non-conditioned rats or in the rats where the consolidation of this fear memory has been inhibited by treatment with escitalopram. This may account for our not finding any difference in activation in the DCbN between the different groups.

A different explanation is related to the stimuli themselves. In a previous study we found a decreased activation in the DCbN directly after panic behaviour caused by dIPAG-stimulation ${ }^{32}$. In the present study the delay between stimulation and sacrifice was 14 hours, in contrast to two hours in the previous study. Therefore we did not expect to see an effect of stimulation since the maximal expression of c-Fos takes place two to four hours after a stimulus ${ }^{48}$. Electrical stimulation is a very strong stimulus. Possibly the conditioned fear is a weaker stimulus, as a result of which group sizes may not have been adequate to disclose a significant effect. This may furthermore account 
for the low and variable signal intensity seen in the sections. The variability in signal intensity was not seen in the cerebellar cortical granule cell layer, therefore it could not be attributed to mere staining quality. There was no significant lateralization across the different treatment groups. This could be related to the suboptimal expression. It is also likely that the effects of dIPAG-stimulation are not confined to just one of the cerebellar hemispheres but have a more generalized effect (as claimed in the DBS background effect theory), since many structures in the cerebrocerebellar loop are (at least partially) bilaterally interconnected ${ }^{9}$.

Although not significant, there is a substantial increase in the amount of c-Fos $\mathrm{ir} / \mathrm{mm}^{2}$ in the DBS dIPAG- buspirone group compared to the sham-buspirone group (from 50 cells $/ \mathrm{mm}^{2}$ in the sham buspirone group to 124 cells $/ \mathrm{mm}^{2}$ in the dIPAGbuspirone group) and a decrease in the amount of c-Fos ir/ $\mathrm{mm}^{2}$ in the dIPAGescitalopram group compared to the sham-escitalopram group (from 68 cells $/ \mathrm{mm}^{2}$ in the sham-escitalopram group to $51 \mathrm{cells} / \mathrm{mm}^{2}$ in the dIPAG-escitalopram group) (Figure 5.2). These changes did not show a correlation with the measured behavioural parameters: freezing and escape behaviour increased in the dIPAG stimulated group as a whole and this effect was normalised only by escitalopram in the stimulated group. A possible explanation could be that there is a mild combined permissive effect of DBS with buspirone treatment on c-Fos expression without any significant change in the measured behavioural parameters. No evidence was found in literature to back up this hypothesis.

In some of the sections we identified hyperintense cytoplasma in the absence of any nuclear staining with c-Fos. This was independent of the intensity of the staining. Some of these cells showed almost isointensity with adjacent c-Fos stained nuclei. This could be due to Fos-like immunoreactivity (Fos-LI) as has previously been described ${ }^{49}$. Possibly there is an influence of deep brain stimulation induced electrochemical changes, altering electrical charges or even inducing cellular alterations considering the trafficking of proteins from diverse intracellular departments.These sparsely distributed cells were seen in only a few sections of the dIPAG stimulated group with escitalopram and sham with saline and escitalopram. Therefore there was no relation with the treatment group, which pleads against an influence of DBS. The number of these cells was too small to perform statistical analysis on. However, a vue microscopy did show that these cells were more often to be found in the lateral regions of the $\mathrm{DCbN}$. This interesting finding could point towards several things. It could indicate that these cells are of a different subset of neurons or that the functional state of these neurons has changed (i.e. different metabolism or a pre-apoptotic state). The exact nature of this phenomenon remains a topic of discussion. 


\section{Conclusion}

In this study we found no significant change in activation of the $\mathrm{DCbN}$ in rats that showed a conditioned fear response induced by dIPAG stimulation 12 hours before testing. We hypothesize that the lack of a significant difference in activation of the DCbN may be related to fear consolidation which takes place after dIPAG stimulation, after which retrieval of behaviour, whether adequate or inadequate, does not lead to a different activation of the DCbN. In our previous research we found deactivation of the DCbN associated with panic behaviour induced by dIPAG-stimulation. The conditioned fear response may have caused smaller changes in cerebellar activation which were not disclosed due to small group sizes. 


\section{References}

1. Manto M. The cerebellum, cerebellar disorders, and cerebellar research--two centuries of discoveries. Cerebellum. 2008;7:505-16.

2. Glickstein M, Strata P, Voogd J. Cerebellum: history. Neuroscience. 2009;162:549-59.

3. Fine EJ, Ionita CC, Lohr L. The history of the development of the cerebellar examination. Semin Neurol. 2002;22:375-84.

4. Pearce JM. Marie-Jean-Pierre Flourens (1794-1867) and cortical localization. Eur Neurol. 2009;61: 311-4.

5. Leiner HC, Leiner AL, Dow RS. Does the cerebellum contribute to mental skills? Behav Neurosci. 1986;100:443-54.

6. Leiner HC, Leiner AL, Dow RS. Cognitive and language functions of the human cerebellum. Trends Neurosci. 1993;16:444-7.

7. Schmahmann JD, Sherman JC. The cerebellar cognitive affective syndrome. Brain. 1998;121 (Pt 4): 561-79.

8. Cantalupo $\mathrm{C}$, Hopkins W. The cerebellum and its contribution to complex tasks in higher primates: a comparative perspective. Cortex. 2010;46:821-30.

9. Allen G, McColl R, Barnard H, Ringe WK, Fleckenstein J, Cullum CM. Magnetic resonance imaging of cerebellar-prefrontal and cerebellar-parietal functional connectivity. Neuroimage. 2005;28:39-48.

10. Middleton FA, Strick PL. Cerebellar projections to the prefrontal cortex of the primate. J Neurosci. 2001;21:700-12.

11. Yamamoto T, Yoshida K, Yoshikawa H, Kishimoto $\mathrm{Y}, \mathrm{Oka} \mathrm{H}$. The medial dorsal nucleus is one of the thalamic relays of the cerebellocerebral responses to the frontal association cortex in the monkey: horseradish peroxidase and fluorescent dye double staining study. Brain Res. 19928; 579:315-20.

12. Cavdar S, Onat FY, Yananli HR, Sehirli US, Tulay C, Saka E, Gürdal E. Cerebellar connections to the rostral reticular nucleus of the thalamus in the rat. J Anat. 2002;201:485-91.

13. Middleton FA, Strick PL. Basal ganglia and cerebellar loops: motor and cognitive circuits. Brain research Brain research reviews. 2000;31:236-50.

14. Dietrichs E, Haines DE, Roste GK, Roste LS. Hypothalamocerebellar and cerebello-hypothalamic projections--circuits for regulating nonsomatic cerebellar activity? Histol Histopathol. 1994;9:603-14.

15. Schmahmann JD, Pandya DN. Prelunate, occipitotemporal, and parahippocampal projections to the basis pontis in rhesus monkey. J Comp Neurol. 1993;337:94-112.

16. Brodal P, Bjaalie JG, Aas JE. Organization of cingulo-ponto-cerebellar connections in the cat. Anat Embryol (Berl). 1991;184:245-54.

17. Schmahmann JD, Pandya DN. Projections to the basis pontis from the superior temporal sulcus and superior temporal region in the rhesus monkey. J Comp Neurol. 1991;308:224-48.

18. Brodal P. The corticopontine projection in the rhesus monkey. Origin and principles of organization. Brain. 1978;101:251-83.

19. Turner BM, Paradiso S, Marvel CL, Pierson R, Boles Ponto LL, Hichwa RD, Robinson RG. The cerebellum and emotional experience. Neuropsychologia. 2007;45:1331-41.

20. Snider RS, Maiti A. Cerebellar contributions to the Papez circuit. J Neurosci Res. 1976;2: 133-46.

21. Sacchetti B, Scelfo B, Strata P. The cerebellum: synaptic changes and fear conditioning. Neuroscientist. 2005;11:217-27.

22. Schmahmann JD. From movement to thought: anatomic substrates of the cerebellar contribution to cognitive processing. Hum Brain Mapp. 1996;4:174-98.

23. Marien P, Engelborghs S, De Deyn PP. Cerebellar neurocognition: a new avenue. Acta Neurol Belg. 2001;101:96-109.

24. Baillieux H, De Smet HJ, Dobbeleir A, Paquier PF, De Deyn PP, Marien P. Cognitive and affective disturbances following focal cerebellar damage in adults: a neuropsychological and SPECT study. Cortex. 2010;46:869-79.

25. Schmahmann JD, Sherman JC. The cerebellar cognitive affective syndrome. Brain. 1998;121:561-79.

26. Schmahmann JD. The role of the cerebellum in cognition and emotion: personal reflections since 1982 on the dysmetria of thought hypothesis, and its historical evolution from theory to therapy. Neuropsychol Rev. 2010;20:236-60. 
27. Wells EM, Walsh KS, Khademian ZP, Keating RF, Packer RJ. The cerebellar mutism syndrome and its relation to cerebellar cognitive function and the cerebellar cognitive affective disorder. Dev Disabil Res Rev. 2008;14:221-8.

28. Baillieux H, De Smet HJ, Paquier PF, De Deyn PP, Marien P. Cerebellar neurocognition: insights into the bottom of the brain. Clin Neurol Neurosurg. 2008;110:763-73.

29. Paulesu E, Frith CD, Frackowiak RS. The neural correlates of the verbal component of working memory. Nature. 1993;362:342-5.

30. de Ribaupierre S, Ryser C, Villemure JG, Clarke S. Cerebellar lesions: is there a lateralisation effect on memory deficits? Acta Neurochir (Wien). 2008;150:545-50; discussion 50.

31. Moers-Hornikx VM, Sesia T, Basar K, Lim LW, Hoogland G, Steinbusch HW, Gavilanes DA, Temel Y, Vles JS. Cerebellar nuclei are involved in impulsive behaviour. Behav Brain Res. 2009;203:256-63.

32. Moers-Hornikx VM, Vles JS, Lim LW, Ayyildiz M, Kaplan S, Gavilanes AW, Hoogland G, Steinbusch HW, Temel Y. Periaqueductal grey stimulation induced panic-like behaviour is accompanied by deactivation of the deep cerebellar nuclei. Cerebellum. 2011; 10:61-9.

33. Desbonnet L, Temel Y, Visser-Vandewalle V, Blokland A, Hornikx V, Steinbusch HW. Premature responding following bilateral stimulation of the rat subthalamic nucleus is amplitude and frequency dependent. Brain Res. 2004;1008:198-204.

34. Moers-Hornikx VM, Vles JS, Tan SK, Cox K, Hoogland G, Steinbusch WM, Temel Y. Cerebellar nuclei are activated by high-frequency stimulation of the subthalamic nucleus. Neurosci Lett. 2011;496:111-5..

35. Sacchetti B, Scelfo B, Strata P. The cerebellum: synaptic changes and fear conditioning. Neuroscientist. 2005;11:217-27.

36. Sacchetti B, Scelfo B, Strata P. Cerebellum and emotional behavior. Neuroscience. 2009;162:756-62.

37. Lim LW, Blokland A, Tan S, Vlamings R, Sesia T, Aziz-Mohammadi M, Visser-Vandewalle V, Steinbusch $\mathrm{HW}$, Schruers K, Temel Y. Attenuation of fear-like response by escitalopram treatment after electrical stimulation of the midbrain dorsolateral periaqueductal gray. Exp Neurol. 2010; 226:293-300.

38. Lino-de-Oliveira C, de Oliveira RM, Padua Carobrez A, de Lima TC, del Bel EA, Guimaraes FS. Antidepressant treatment reduces Fos-like immunoreactivity induced by swim stress in different columns of the periaqueductal gray matter. Brain Res Bull. 2006;70:414-21.

39. Temel Y, Boothman LJ, Blokland A, Magill PJ, Steinbusch HW, Visser-Vandewalle V, Sharp T. Inhibition of 5-HT neuron activity and induction of depressive-like behavior by high-frequency stimulation of the subthalamic nucleus. Proc Natl Acad Sci U S A. 2007;104:17087-92.

40. Lim LW, Blokland A, Visser-Vandewalle V, Vlamings R, Sesia T, Steinbusch H, Schruers K, Griez E, Temel $Y$. High-frequency stimulation of the dorsolateral periaqueductal gray and ventromedial hypothalamus fails to inhibit panic-like behaviour. Behav Brain Res. 2008;193:197-203.

41. Tan S, Vlamings R, Lim L, Sesia T, Janssen ML, Steinbusch HW, Visser-Vandewalle V, Temel Y. Experimental deep brain stimulation in animal models. Neurosurgery. 2010;67:1073-9; discussion80.

42. Assie $M B$, Lomenech $H$, Ravailhe $V$, Faucillon V, Newman-Tancredi A. Rapid desensitization of somatodendritic 5-HT1A receptors by chronic administration of the high-efficacy 5-HT1A agonist, F13714: a microdialysis study in the rat. Br J Pharmacol. 2006;149:170-8.

43. Bondi CO, Rodriguez G, Gould GG, Frazer A, Morilak DA. Chronic unpredictable stress induces a cognitive deficit and anxiety-like behavior in rats that is prevented by chronic antidepressant drug treatment. Neuropsychopharmacology. 2008;33:320-31.

44. Burghardt NS, Sullivan GM, McEwen BS, Gorman JM, LeDoux JE. The selective serotonin reuptake inhibitor citalopram increases fear after acute treatment but reduces fear with chronic treatment: a comparison with tianeptine. Biol Psychiatry. 2004;55:1171-8.

45. Sato H, Skelin I, Debonnel G, Diksic M. Chronic buspirone treatment normalizes open field behavior in olfactory bulbectomized rats: assessment with a quantitative autoradiographic evaluation of the 5HT1A binding sites. Brain Res Bull. 2008;75:545-55.

46. Lim LW, Temel Y, Visser-Vandewalle V, Steinbusch H, Schruers K, Hameleers R, Esquivel G, Griez E, Blokland A. Effect of buspirone on the behavioral regulation of rats in low versus high anxiety conditions. Arzneimittelforschung. 2008;58:269-76.

47. Watson GPC. The Rat Brain in Stereotaxic Coordinates. 4th edition ed: Academic Press; 1998.

48. Smith MA, Banerjee S, Gold PW, Glowa J. Induction of c-fos mRNA in rat brain by conditioned and unconditioned stressors. Brain Res. 1992;578:135-41. 
49. Duan XQ, Wu SL, Li T, Liang JC, Qiou JY, Rao ZR, Ju G. Expression and significance of three types of Fosimmunoreactive cells after gamma knife irradiation of the forebrain in the rat. Neurosci Res. 1999;33:99-104. 



\section{Chapter 6}

Sustained reduction of cerebellar activity in experimental epilepsy

K Rijkers, VMP Moers-Hornikx, RJ Hemmes, MW Aalbers, Y Temel, JSH Vles, G Hoogland In preparation 


\section{Abstract}

Clinical and experimental evidence suggests a role for the cerebellum in seizure control, while no data are available on cerebellar activity between seizures. We hypothesized that interictal regional activity of the deep cerebellar nuclei is reduced in epilepsy, and tested this in an animal model by using $\triangle$ FosB and cytochrome oxidase (COX) (immuno)histochemistry. The expression of these two markers of neuronal acivity was analysed in the dentate (DN), interpositus (IN) and fastigial (FN) nucleus of the cerebellum of fully amygdala kindled rats that were sacrificed 48 hours after their last seizure.

Cerebellar nuclei of kindled rats exhibited 22 to $28 \%$ less $\Delta F$ osB immunopositive cells than their respective counterpart in sham controls $(p<0.05)$. The density of $\Delta F o s B$ immunopositive cells correlated negatively with the pre-kindling afterdischarge threshold (DN: $r=-0.726 ; \mathrm{IN}$ : $r=-0.635 ; F N: r=-0.664, p<0.05$ ) and the change in afterdischarge threshold (DN: $r=-0.664 ; I N$ : $r=-0.625 ; p<0.05)$. COX expression in the IN and $\mathrm{FN}$ of kindled animals was reduced by $32 \%$ compared to respective control values $(p<0.05)$.

These results indicate that an epileptogenic state is characterized by decreased activity of deep cerebellar nuclei, especially the FN. Possible consequences may include a decreased activation of the thalamus, contributing to further seizure spread. Restoration of FN activity by low frequency electrical stimulation is suggested as a possible treatment option in chronic epilepsy. 


\section{Introduction}

The cerebellum plays a crucial role in the coordination and control of motor behaviour and cognitive processing ${ }^{1}$. Studies in epilepsy patients and animals models of epilepsy have

demonstrated that the cerebellum takes part in the epileptogenic network as well. First of all, case reports have illustrated that the cerebellum can harbour the epileptic focus ${ }^{2-4}$. Furthermore, SPECT and $\mathrm{FMRI}$ studies in epilepsy patients have revealed that cerebellar blood flow and blood oxygen level-dependant (BOLD) signal, respectively, increase during secondary generalisation of a partial seizure ${ }^{5-11}$. In rats, cerebellar glucose utilization increases upon administration of proconvulsant drugs ${ }^{12}$.

At the cellular level it has been hypothesized that this ictally increased activity reflects increased GABA-ergic Purkinje cell firing ${ }^{13}$. Purkinje cells provide the purely inhibitory,output of the cerebellar cortex ${ }^{14}$. These inhibitory projections modify the output of the cerebellum through the deep cerebellar nuclei, the dentate nucleus (DN), interpositus nucleus (IN) and fastigial nuclei (FN), which in turn project to the thalamus. This way, the cerebellum regulates activity of neurons elsewhere in the brain.

Activation of the Purkinje fibers by electrical stimulation of the cerebellar cortex has anticonvulsive effects in animal studies ${ }^{15,16}$. It has therefore been investigated as an anticonvulsive therapy in humans as well, with varying results ${ }^{17-20}$. The deep cerebellar nuclei are interconnected and therefore their output is affected by more than Purkinje cell firing alone ${ }^{21}$. Modification of this deep cerebellar network by electrical stimulation of the $\mathrm{DN}^{22-24}$ and $\mathrm{FN}^{25,26}$ has been performed in an attempt to treat epilepsy patients.

In summary, seizures are associated with increased activity of the Purkinje cells. Since these cells inhibit the deep cerebellar nuclei, it is likely that epilepsy is associated with decreased activity of these nuclei. It is not clear whether the different deep cerebellar nuclei are all inhibited to the same extent. The aim of the current study was therefore to assess the regional distribution of interictal neuronal activity in the deep cerebellar nuclei. To this end, we submitted cerebellar sections from fully amygdala kindled rats to (immuno)histochemistry of neuronal activity markers $\triangle$ FosB and cytochrome C oxidase (COX, a.k.a. complex IV), and quantified their expression in the DN, IN and FN.

\section{Materials and methods}

\section{Animals}

Experimental procedures were carried out in 12-weeks old male Sprague-Dawley rats (Harlan, Horst, The Neterhlands). Animals were housed under controlled conditions, a 12 hour light/dark cycle (lights on $7 \mathrm{am}$ ), background noise, and food and water ad libitum. Adequate measures were taken to minimize pain and discomfort. All 
experimental procedures were approved by the animal ethics committee of Maastricht University and complied with governmental legislation.

\section{Amygdala kindling}

\section{Electrode placement}

For amygdala kindling, rats were stereotactically implanted with an electrode in the left basolateral amygdala. The surgical procedure was performed under general isoflurane anesthesia ( $5 \%$ for induction and $2.5 \%$ for maintenance). Perioperatieve pain was minimized by administering $0.1 \mathrm{ml}$ buprenofrinehydro-chloride (Temgesic ${ }^{\circledR}$, ScheringPlough Inc., Amstelveen, The Netherlands) subcutaneously 30 minutes before surgery. Rats were stereotactically implanted (Dual Manipulator Lab Standard Stereotact, Stoelting Inc., Wood Dale, USA) with a set of electrode designed and manufactured by the department of Instrument Development, Engineering and Evaluation of Maastricht University in collaboration with prof. dr. Y. Temel (Maastricht University Medical Center, Maastricht, The Netherlands). An electrode set consisted of a bipolar stimulating/recording electrode that was implanted in the left basolateral amygdala (coordinates relative to bregma: $2.5 \mathrm{~mm}$ posteriorly, $4.8 \mathrm{~mm}$ laterally, and $9.6 \mathrm{~mm}$ ventrally ${ }^{27}$ ), and three monopolar stainless steel electrodes that were implanted in the cortex at $1 \mathrm{~mm}$ depth. One cortical electrode was used for EEG, one for reference, and one for ground. Connectors for the kindling/EEG electrodes were fixed on the skull using dental acrylic.

\section{Afterdischarge threshold}

Ten days after surgery the pre-kindling afterdischarge threshold (pre-KADT) was assessed in all rats by stimulating the amygdala with a series of pulses of increasing intensity starting at $10 \mu \mathrm{A}$ ( 2 seconds, $50 \mathrm{~Hz}, 0.2 \mathrm{~ms}$ blockpulse). Stimuli were delivered through a WPI Accupulser A310 connected to a WPI Stimulus Isolation Unit A360 (World Precision Instruments, Sarasota, FL, USA). EEG registrations from the amygdala and cortical electrodes were recorded from $1 \mathrm{~min}$ before the kindling stimulus till the end of the behavioural seizure using a Vangard system (Vangard Systems, Cleveland Clinics Foundation, Cleveland, USA). Recordings were made with a sample frequency of $200 \mathrm{~Hz}$, a frequency band of $0.5-70 \mathrm{~Hz}$ and a $50 \mathrm{~Hz}$ notch filter. The afterdischarge threshold was defined as the stimulus amplitude necessary to elicit a two-second discharge with a high frequency and high voltage. One day after the last kindling stimulus, a post-kindling afterdischarge threshold (post-KADT) was assessed. The difference between pre-KADT and post-KADT ( $\triangle \mathrm{ADT}$ ) was calculated by subtracting the respective values and was considered an indirect measure of excitability. 


\section{Amygdala kindling}

Amygdala kindling started two weeks after surgery by administering two stimulations per day with and interstimulus interval of at least six hours. Each stimulus lasted two seconds and consisted of a $50 \mathrm{~Hz}, 400 \mu \mathrm{A}, 0.2 \mathrm{~ms}$ blockpulses. Seizure severity was assessed based on Racine's scale ${ }^{28}$ (i.e. stage 1: freezing; stage 2: head nodding, blinking; stage 3: unilateral forelimb clonus; stage 4: bilateral forelimb clonus, rearing; stage 5: falling). Fully kindled animals were defined as animals that displayed a stage five seizure upon five consecutive amygdala stimulations. These fully kindled animals were subsequently stimulated once per day for two more weeks. Kindling rate was defined as the number of stimuli needed to reach the fully kindled state. Sham animals underwent the same surgical procedure and aftersdischarge assessments, but were not subjected to daily stimulation.

\section{Histological processing}

Two days after the last seizures the rats received an overdose of pentobarbital (Nembutal), followed by perfusion with tyrode buffer (in mM: $136.9 \mathrm{NaCl}, 2.7 \mathrm{KCl}$, $0.2 \mathrm{MgCl}_{2}, 11.9 \mathrm{NaHCO}_{3}, 0.3 \mathrm{NaH}_{2} \mathrm{PO}_{4}, 5.0$ glucose, equilibrated with $5 \% \mathrm{CO}_{2} / 95 \% \mathrm{O}_{2}$ ), and then with $4 \%$ paraformaldehyde dissolved in $0.1 \mathrm{M}$ phosphate buffer, $\mathrm{pH}$ 7.6. The brains were removed, postfixed in the same fixative $\left(4^{\circ} \mathrm{C}, 24\right.$ hours), rapidly frozen using $\mathrm{CO}_{2}$, and stored at $-80^{\circ} \mathrm{C}$ until sections were cut.

Coronal $10 \mu \mathrm{m}$ serial sections from the level of the central canal-fourth ventricle junction (13.6 $\mathrm{mm}$ posteriorly to bregma according to Paxinos ${ }^{27}$ ) caudally to the inferior colliculus (located $9.6 \mathrm{~mm}$ posteriorly to bregma) were cut on a cryostat, mounted on gelatin-coated glass slides, and stored at $-20^{\circ} \mathrm{C}$ until they were processed for (immune)histochemistry. To minimize staining-to-staining variations, staining experiments always included an equal amount of control and kindled samples.

\section{$\triangle$ FosB immunohistochemistry}

Sections were rinsed in Tris-buffered saline containing $0.3 \%$ Trition (TBS-T), and then incubated $\left(4^{\circ} \mathrm{C}, 48\right.$ hours) with polyclonal rabbit-anti-mouse for $\mathrm{B}$ antibody (sc-48, Santa Cruz Biotechnology Inc., Santa Cruz, CA, USA) diluted 1:250 in TBS-T containing $0.5 \%$ bovine serum albumin. Next, sections were rinsed in TBS-T, and incubated (room temperature, overnight) with donkey-anti-rabbit biotinylated antibody (Jackson ImmunoResearch Laboratories Inc., West Grove, PA, USA) diluted 1:400 in TBS-T containing $0.5 \%$ bovine serum albumin. Sections were then rinsed with TBS-T, incubated for two hours in avidin/biotin diluted 1:800 (Vectastain ABC-kit, Vector Laboratories Inc., Burlingame, CA, USA), and visualised by 3.3'-diaminobenzidine (DAB) containing $\mathrm{NiCl}_{2}$. Finally, slides were dehydrated and coverslipped. 


\section{COX histology}

COX was histochemically detected as described previously ${ }^{29,30}$. Briefly, sections were air dried and subsequently incubated $\left(37^{\circ} \mathrm{C}\right.$, four hours) in the dark in a nickel enhanced 0.1M 4-(2-hydroxyethyl)-1-piperazineethanesulfonic acid (HEPES) solution $\left(1 \% \mathrm{NiCl}_{2}, \mathrm{pH} 7.4\right)$ containing $0,0224 \%$ cytochrome Coxidase, $0,115 \% \mathrm{DAB}$, and $4,5 \%$ sucrose. The reaction was stopped by transferring the sections to an icecold pH neutral buffered $4 \%$ paraformaldehyde medium for ten minutes. Finally, slides were dehydrated and coverslipped.

\section{Quantification of stainings}

The dentate nucleus (DN), interpositus nucleus (IN) and fastigial nucleus (FN) were photographed in all sections at $4 X$ magnification using an Olympus AX70 bright-field microscope with an Olympus DP70 camera (analySIS Imaging System, Münster, Germany). A mean of 10 sections per animal for the $\Delta$ fosBstaining and 16 sections per animals for the COX-staining were analysed. The deep cerebellar nuclei were easily identifiable due to the contrast between nuclei and surrounding white matter, allowing delineation using the freehand drawing function in ImageJ (version 1.43). Artifacts were excluded from analysis.

Cells showing $\Delta$ fosB immunoreactivity in the nucleus, cytoplasm, or both were counted by and observer that was blinded for the treatment and expressed as number of immunopositive cells per square millimeter. In total, 84 sections from 7 kindled animals, and 72 sections from 9 sham animals were analysed. Cytochrome oxidase staining was analysed by optical density (OD) using ImageJ. For each nucleus, the OD value was corrected for background activity by subtracting the OD of the adjacent white matter, resulting in negative values as ImageJ applies an inverted grey value scale. In total, 92 stained sections from 6 kindled animals and 101 sections from 6 sham animals were analysed.

\section{Statistical analysis}

Shapiro-Wilk test was performed as test for normal distribution of data. Group differences from normally distributed data were tested by a two-tailed independent samples t-test was used, while data that were not normally distributed were analysed using a Mann-Whitney test. Data were pooled when there was no significant difference between the left and right hemisphere. Within the dataset of one nucleus from one rat, an outlier was defined as a value that deviated more than 2 standard deviations from the mean. This was corrected for by adjusting the value to maximal 2 standard deviations. If the averaged values for one nucleus exceeded 2 standard deviations from the mean of this nucleus in the treatment group it was excluded from the analysis. The correlation between the expression of neuronal activity markers and the kindling parameters (pre-KADT, post-KADT, $\triangle \mathrm{ADT}$ and kindling rate) was tested by bivariate analysis and presented as Pearson's correlation coefficient. Data are expressed as 
mean \pm standard error of the mean (SEM). $p<0.05$ was considered statistically significant.

\section{Results}

There were no significant differences in $\triangle$ FosB immunopositive cell densities or in COX grey values between left and right cerebellar nuclei in shams nor in kindled animals. Therefore, to increase the power of the analysis, values from both hemispheres were pooled.

\section{$\triangle F O s B$}

Density of $\triangle$ FosB immunoreactive ( $\triangle$ FosB-ir) cells was lower in all three cerebellar nuclei of kindled animals than in shams (DN 108.5 \pm 12.4 vs $152.1 \pm 7.7$; FN 87.2 \pm 7.8 vs. $115.5 \pm 2.2$ and IN $91.3 \pm 9.1$ vs. $116.2 \pm 3.5 ; p<0.05$ for $D N$ and $I N ; p<0.01$ for FN), see Figure 6.1. In both sham and kindled animals, the DN contained a significantly $(p<0.05)$ higher $\Delta$ FosB-ir cell density (shams 152.1 \pm 7.7 ; kindled 108.5 \pm 12.4 ) than FN (shams 115.5 \pm 2.2 ; kindled 87.2 \pm 7.8 ) and IN (shams 116.2 \pm 3.5 ; kindled 91.3 \pm 9.1 ).

In kindled animals, $\triangle$ FosB-ir cell density was negatively correlated with pre-KADT in all three nuclei (DN: $r=-0.726$; IN: $r=-0.635$; $F N$ : $r=-0.664, p<0.05$ ). Similarly there was a negative correlation between $\triangle$ FosB-ir cell density and $\triangle A D T$ in the DN and IN of kindled animals ( $D N$ : $r=-0.664$; IN: $r=-0.625, p<0.05$ ), meaning that a lower $\triangle$ FosB-ir cell density is associated with a higher pre-KADT and a larger $\triangle$ ADT (see Figure 6.2). No correlation was found between $\triangle$ FosB immunopositive cell density and post-KADT or kindling rate (data not shown).

$\Delta$ FosB immunohistochemical staining was not confined to the cell nucleus, as would be expected from a nuclear staining, but was seen in the cytoplasm as well (see Figure 6.3). This ' $\Delta$ FosB-like immunoreactivity' was almost exclusively found in the most lateral regions of the deep cerebellar nuclei, and was particularly observed in the DN. 

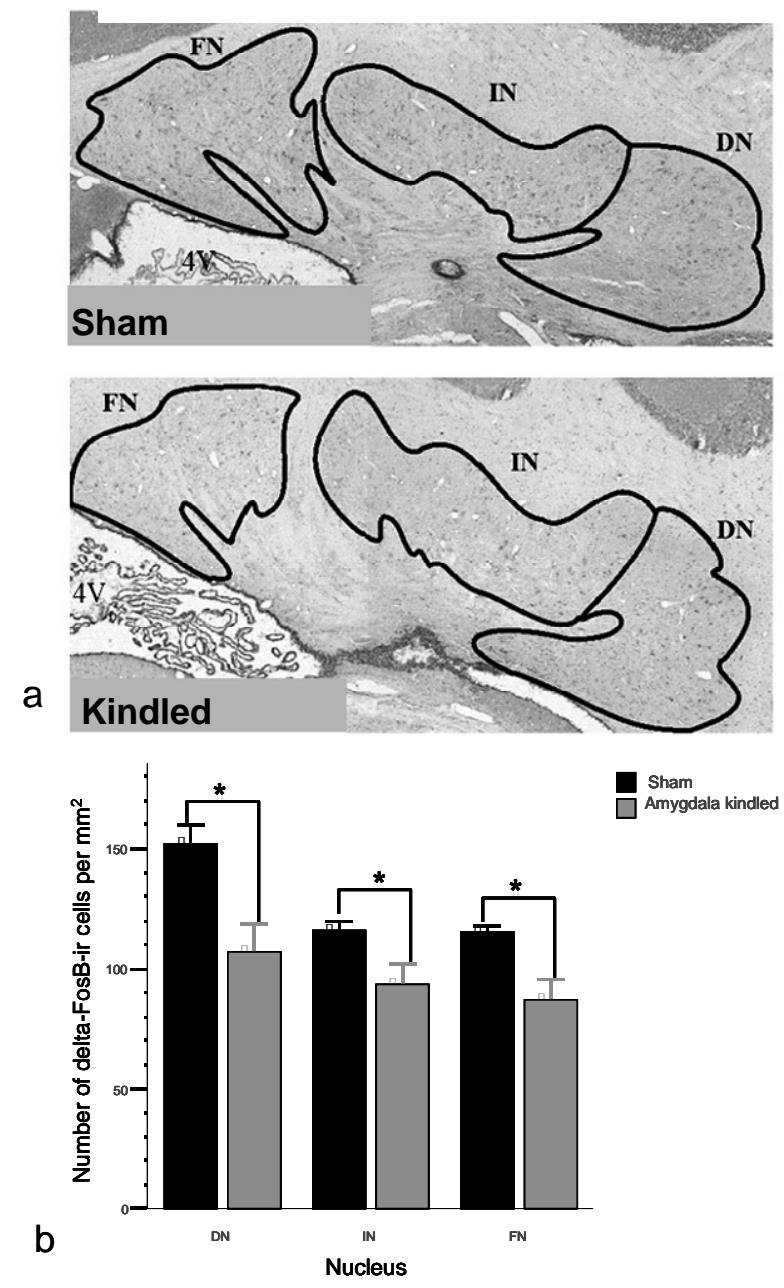

Figure 6.1 Amygdala kindling is associated with a reduced cerebellar $\Delta$ FosB expression. (a) Representative photomicrographs (4x magnification) showing $\Delta$ FosB immunoreactivity in the deep cerebellar nuclei of a sham and a fully amygdala kindled rat. Note the overall reduction in $\Delta$ FosB immunoreactivity in the kindled rat, suggesting a general decrease in neuronal activity. Coronal sections were taken at bregma level -11.30 . $4 \mathrm{~V}$ : fourth ventricle; DN: dentate nucleus; FN: fastigial nucleus; IN: interpositus nucleus. (b) Number of $\Delta$ FosB immunoreactive (ir) cells/mm2 in the deep cerebellar nuclei. Data represent mean \pm SEM of 72 sections from 9 sham animals and 84 sections from 7 kindled animals. ${ }^{*} p<0.05$. 

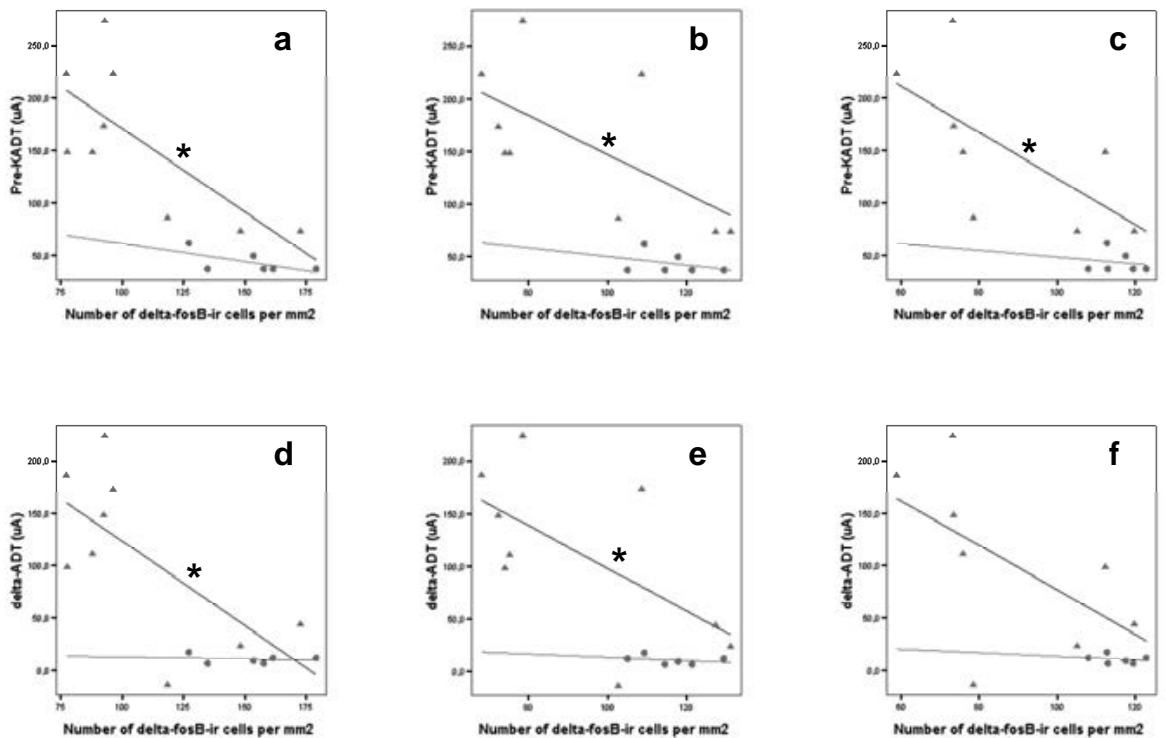

Figure 6.2 The neural activity of the deep cerebellar nuclei correlates negatively with seizure threshold. Correlations between $\triangle F o s B$ immunoreactivity in the nuclei ( $\mathbf{a}$ and $\mathbf{d}$ : dentate; $\mathbf{b}$ and $\mathbf{e}$ : interpositus; $\mathbf{c}$ and $\mathbf{f}$ : fastigial) and the pre-kindling afterdischarge threshold (a-c) or the change in afterdischarge threshold (d-f). -: sham; $\mathbf{A}$ amygdala kindled rats; ${ }^{*} \mathrm{p}<0.05$.

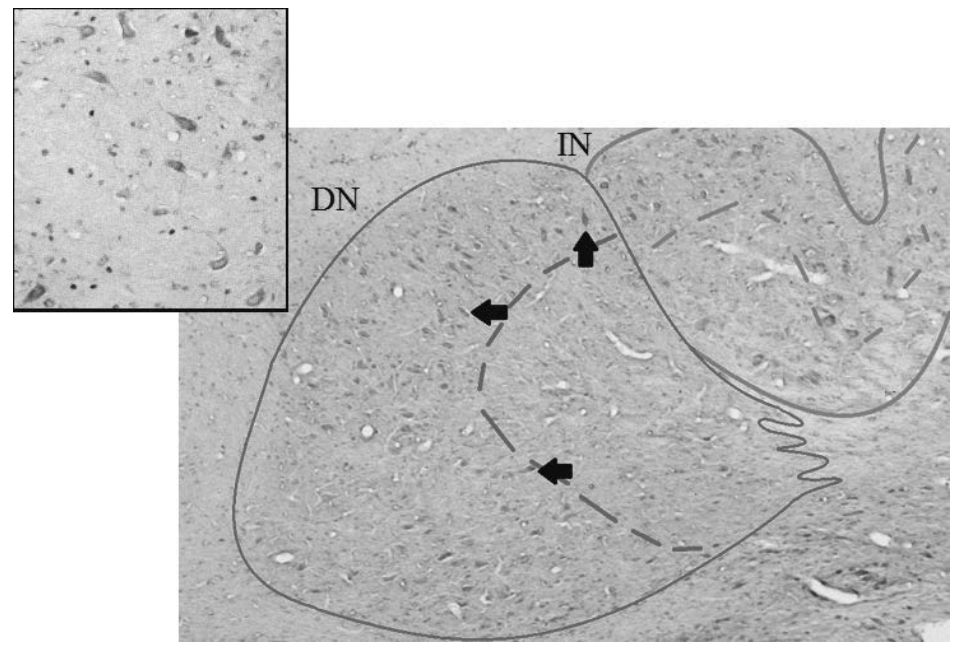

Figure 6.3 Photomicrograph (10x magnification) showing the distribution of $\Delta$ FosB immunoreactive cells in the lateral deep cerebellar nuclei. The cytoplasmic staining of $\Delta$ FosB is seen in relatively large cells (arrows) that are almost exclusively localized in the outer regions of the nuclei. These cells are seen in the deep cerebellar nuclei of both sham and amygdala kindled rats. The inset is a 40x magnification showing the cytoplasmic staining in more detail. DN: dentate nucleus; IN: interpositus nucleus. 


\section{$\operatorname{cox}$}

Kindled animals expressed significantly less COX than shams in both FN and IN (FN: $-32.7 \pm 3.4$ vs. $21.9 \pm 2.8, p<0.05$; IN: $-47.7 \pm 4.2$ vs. $-32.4 \pm 4.8, p<0.05$, see Figure 6.4 ). In the DN, COX expression was also lower in the kindled animals than in the shams, but this difference was not statistically significant $(-39.4 \pm 5.5$ vs. $-55.5 \pm 5.2 ; p=0.078)$. COXexpression showed no significant correlation with any of the kindling parameters.
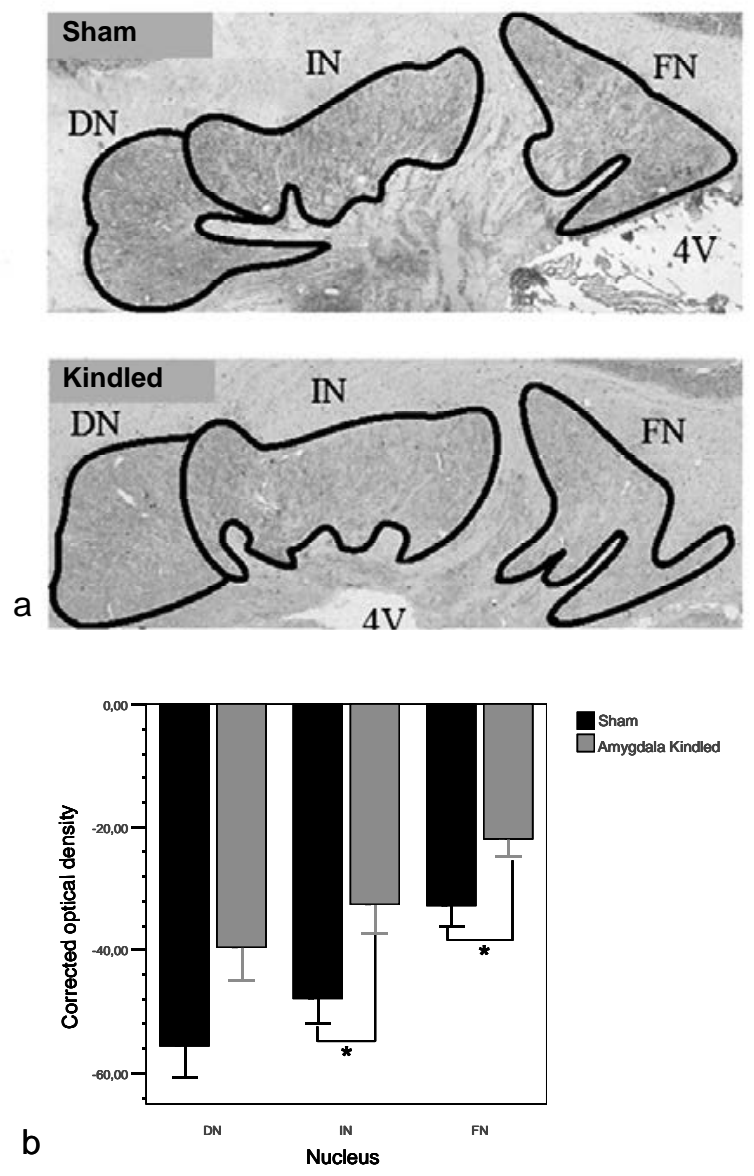

Figure 6.4 Amygdala kindling is associated with a reduced cerebellar cytochrome oxidase (COX) expression. (a) Representative photomicrographs (4x magnification) showing COX activity in the deep cerebellar nuclei of a sham and a fully amygdala kindled rat. Note the overall reduction in COX activity in the kindled rat, suggesting a general decrease in neuronal activity. Coronal sections were taken at bregma level -11.30 . 4V: fourth ventricle; DN: dentate nucleus; IN: interpositus nucleus; FN: fastigial nucleus. (b) Corrected optical density of COX activity in the deep cerebellar nuclei. The optical density was corrected for background staining by subtracting the optical density of the adjacent white matter, leading to a negative value. Data represent mean \pm SEM of 101 sections from 6 sham animals and 92 sections from 7 kindled animals. $* p<0.05$. 


\section{Discussion}

In this study, we have identified the pattern of interictal neuronal activity in the deep cerebellar nuclei of chronically epileptic rats. We were able to confirm our hypothesis that chronically epileptic rats show less neuronal activity in these nuclei than shams, reflected by a significantly lower density of $\triangle$ FosB immunopositive cells in the IN, FN and DN and by a significantly lower expression of COX in the FN and IN. We have furthermore shown that a lower level of neuronal activity is associated with a higher pre-KADT and a higher $\triangle$ ADT.

\section{Methodological considerations}

All experiments were carried out in fully kindled animals that were sacrificed 48 hours after their last kindled seizure. This means that we investigated interictal changes in a chronic epilepsy model. Data on interictal cerebellar activity in humans are scarce and are indicative of hypometabolism on $\mathrm{PET}^{31}$ and $\mathrm{SPECT}^{32}$. Epileptic dogs did not show any change in cerebellar metabolism interictally ${ }^{33}$. These imaging studies mainly estimate cerebellar cortical activity, while the deep cerebellar nuclei are not evaluated this way. It is therefore not possible to compare our data with in vivo imaging data.

Some areas of the brain are more involved in generalized tonic-clonic seizures than others ${ }^{9,34-36}$. The degree of involvement can be immunohistochemically determined by markers for immediately early genes, such as c-fos, FosB and $\Delta$ FosB. C-Fos and FosB peak at 2 and 6 hours after a stimulus, respectively. $\Delta$ FosB is a highly stable FosB isoform that persist in the brain for several weeks after an initial stimulus ${ }^{37}$. Since the kindling protocol involves repetitive stimuli with interstimulus intervals of more than six hours, we chose to determine the $\triangle$ FosB immunopositive cell density as a marker of neuronal activation. To the best of our knowlegde, no data are availabe in literature on basal cerebellar $\Delta$ FosB levels, nor on cerebellar $\Delta$ FosB expression in epilepsy. Our results in sham rats reveal that basal expression of $\Delta \mathrm{FosB}$ is present in all deep cerebellar nuclei, and that among the deep nuclei, the DN contains the highest density of $\triangle$ FosB immunopositive cells. The DN receives input from the more lateral parts of the cerebellar cortex while IN and FN receive input from the vermian cortex (for a review see Voogd et $a .^{38}$ ). Our data suggest that in shams, the vermian cortex has a stronger inhibitory effect on its target nuclei than the cortex of the cerebellar hemispheres.

Furthermore, there are three types of Fos immunoreactivity: nuclear, cytoplasmic, or both ${ }^{39}$. We found cells with nuclear $\Delta$ FosB immunoreactivity, but also several cells with cytoplasmic staining. This phenomenon has been described for the hippocampus, dentate gyrus and amygdala by others ${ }^{40}$, and appears to be present in the deep cerebellar nuclei as well.

COX is a large transmembrane protein located in mitochondria. It transforms redox energy from the oxidative respiratory chain into a proton-motive force across 
the mitochondrial membrane. In this process it receives an electron from four cytochrome c proteins and donates these electrons to free oxygen molecules, thereby converting them to water ${ }^{41,42}$. Under physiological conditions, COX is involved in the cellular energy supply, while in the process of seizure-induced neurodegeneration it plays a role in the activation of pro-apoptotic pathways ${ }^{12,43-47}$. Here, we used COX as a marker of oxidative metabolism. COX activity has been shown in the rat cerebellum previously (e.g. by using spectrophotometry and immunoblot ${ }^{48}$ ), but cerebellar COX expression has not been evaluated before using COX histology. On the other hand, COX histology has been used before to quantify the degree of cerebral oxidative metabolism after electrical stimulation in the rat ${ }^{30}$.

\section{Epilepsy affects activity of the deep cerebellar nuclei}

We have shown that kindled animals expressed a lower $\triangle \mathrm{FosB}$ immunopositive cell density than shams in all three cerebellar nuclei. Neurons in the FN showed the largest decrease, followed by those in the IN. Our COX data indicate as well that FN and IN are more affected by kindling than the DN (see Figure 6.4).

Apparently, amygdala kindling decreases FN activity more than it reduces the activity of DN and IN. This may be the result of direct antidromic effects of amygdala kindling, bearing in mind the possible anatomical connection between the FN and the amygdala ${ }^{49}$. These connections have been suggested to exist based on several findings. First, behavioural changes are observed following low frequency stimulation of the $\mathrm{FN}^{50}$ or lesioning of the deep cerebellar nuclei ${ }^{51}$. Second, electrophysiological studies have shown that both low- ${ }^{50}$ and higher frequency stimulation ${ }^{49}$ of the FN evokes responses in hippocampus and amygdala bilaterally. Third, lesioning the FN leads to degeneration of synaptic terminals in the amygdala bilaterally ${ }^{49}$.

Next to the possible antidromic effect of amygdala kindling on the cerebellum, activity of the deep cerebellar nuclei may have been affected by the generalized seizures that the animals in this model suffer from, since chronic epilepsy with generalized seizures in humans is also associated with cerebellar changes ${ }^{52,53}$. Decreased activity of the deep cerebellar nuclei may therefore have been secondary to multiple seizures. Output of the $\mathrm{FN}$ is putatively glutamatergic ${ }^{54}$, and decreased activity of the FN may lead to a decreased activation of its most important target structure, the thalamus. This may facilitate further spread of seizures, since the thalamus has been shown to play an important role in seizure control ${ }^{55,56}$

The FN is of particular interest with regard to the treatment of epilepsy since low frequency stimulation of this nucleus as well as stimulation of the vermian cortex (that projects to the FN) are both anticonvulsive ${ }^{16,26}$, while GABA agonist injections into the FN significantly decrease seizure threshold ${ }^{57}$. Furthermore, complete destruction of either the FN is proconvulsive, while partial destruction inhibits seizures ${ }^{58}$. In addition, FN lesions abolish the anticonvulsive effects of vermis stimulation ${ }^{59}$. On the other hand, low frequency stimulation of the DN does not affect seizure activity ${ }^{22}$ and GABAergic injections into the DN do not affect seizures ${ }^{57}$. 


\section{Conclusion}

In our study we confirmed our hypothesis that the deep cerebellar nuclei are hypoactive in chronic epilepsy. This decrease was most pronounced in the FN. Functionally located between limbic system, where seizures often arise, and thalamus, where seizures are propagated, the FN is an interesting target for epilepsy treatment; we hypothesize that low frequency stimulation of the FN restores its activity and thereby its control over thalamus function, explaining anticonvulsive effects of this treatment. 


\section{References}

1. Schmahmann JD. Disorders of the cerebellum: ataxia, dysmetria of thought, and the cerebellar cognitive affective syndrome. J Neuropsychiatry Clin Neurosci. 2004;16:367-78.

2. Mesiwala AH, Kuratani JD, Avellino AM, Roberts TS, Sotero MA, Ellenbogen RG. Focal motor seizures with secondary generalization arising in the cerebellum. Case report and review of the literature. J Neurosurg. 2002;97:190-6.

3. Delande O, Rodriguez D, Chiron C, Fohlen M. Successful surgical relief of seizures associated with hamartoma of the floor of the fourth ventricle in children: report of two cases. Neurosurgery. 2001;49:726-30; discussion 30-1.

4. Gan YC, Connolly MB, Steinbok P. Epilepsy associated with a cerebellar arachnoid cyst: seizure control following fenestration of the cyst. Child's nervous system : ChNS : official journal of the International Society for Pediatric Neurosurgery. 2008;24:125-34.

5. Gale K. Subcortical structures and pathways involved in convulsive seizure generation. J Clin Neurophysiol. 1992;9:264-77.

6. Iadecola C, Yang G, Ebner TJ, Chen G. Local and propagated vascular responses evoked by focal synaptic activity in cerebellar cortex. J Neurophysiol. 1997;78:651-9.

7. Yang G, ladecola C. Activation of cerebellar climbing fibers increases cerebellar blood flow: role of glutamate receptors, nitric oxide, and cGMP. Stroke. 1998;29:499-507; discussion -8.

8. Norden $A D$, Blumenfeld $H$. The role of subcortical structures in human epilepsy. Epilepsy Behav. 2002;3:219-31.

9. Blumenfeld H, Varghese GI, Purcaro MJ, Motelow JE, Enev M, McNally KA, Levin AR, Hirsch LJ, Tikofsky $R$, Zubal IG, Paige AL, Spencer SS. Cortical and subcortical networks in human secondarily generalized tonic-clonic seizures. Brain. 2009;132(Pt 4):999-1012.

10. Auer T, Veto K, Dóczi T, Komoly S, Juhos V, Janszky J, Schwarcz A. Identifying seizure-onset zone and visualizing seizure spread by fMRI: a case report. Epileptic Disord. 2008;10:93-100.

11. Won JH, Lee JD, Chung TS, Park CY, Lee BI. Increased contralateral cerebellar uptake of technetium99m-HMPAO on ictal brain SPECT. J Nucl Med. 1996;37:426-9.

12. Ableitner A, Herz A. Changes in local cerebral glucose utilization induced by the beta-carbolines FG 7142 and DMCM reveal brain structures involved in the control of anxiety and seizure activity. J Neurosci. 1987; 7:1047-55.

13. Salgado-Benitez A, Briones R, Fernandez-Guardiola A. Purkinje cell responses to a cerebral penicillininduced epileptogenic focus in the cat. Epilepsia. 1982;23:597-606.

14. Ito M, Yoshida M, Obata K, Kawai N, Udo M. Inhibitory control of intracerebellar nuclei by the purkinje cell axons. Exp Brain Res. 1970;10:64-80.

15. Bantli H, Bloedel JR, Anderson G, McRoberts R, Sandberg E. Effects of stimulating the cerebellar surface on the activity in penicillin foci. J Neurosurg. 1978;48:69-84.

16. Godlevskii LS, Stepanenko KI, Lobasyuk BA, Sarakhan EV, Bobkova LM. The effects of electrical stimulation of the paleocerebellar cortex on penicillin-induced convulsive activity in rats. Neurosci Behav Physiol. 2004;34:797-802.

17. Cooper IS, Amin I, Riklan M, Waltz JM, Poon TP. Chronic cerebellar stimulation in epilepsy. Clinical and anatomical studies. Arch Neurol. 1976;33:559-70.

18. Van Buren JM, Wood JH, Oakley J, Hambrecht F. Preliminary evaluation of cerebellar stimulation by double-blind stimulation and biological criteria in the treatment of epilepsy. J Neurosurg. 1978;48:40716.

19. Wright GD, McLellan DL, Brice JG. A double-blind trial of chronic cerebellar stimulation in twelve patients with severe epilepsy. J Neurol Neurosurg Psychiatry. 1984;47:769-74.

20. Davis R, Emmonds SE. Cerebellar stimulation for seizure control: 17-year study. Stereotact Funct Neurosurg. 1992;58:200-8.

21. Gould BB, Graybiel AM. Afferents to the cerebellar cortex in the cat: evidence for an intrinsic pathway leading from the deep nuclei to the cortex. Brain Res. 1976;110:601-11.

22. Chkhenkeli SA, Sramka M, Lortkipanidze GS, Rakviashvili TN, Bregvadze ESh, Magalashvili GE, Gagoshidze TSh, Chkhenkeli IS. Electrophysiological effects and clinical results of direct brain stimulation for intractable epilepsy. Clin Neurol Neurosurg. 2004; 106:318-29. 
23. Sramka M, Chkhenkeli SA. Clinical experience in intraoperational determination of brain inhibitory structures and application of implanted neurostimulators in epilepsy. Stereotact Funct Neurosurg. 1990;54-55:56-9.

24. Sramka M, Fritz G, Galanda M, Nadvornik P. Some observations in treatment stimulation of epilepsy. Acta Neurochir (Wien). 1976(23 Suppl):257-62.

25. Hablitz JJ, Rea G. Cerebellar nuclear stimulation in generalized penicillin epilepsy. Brain Res Bull. 1976;1:599-601.

26. Wang S, Wu DC, Ding MP, Li Q, Zhuge ZB, Zhang SH, Chen Z. Low-frequency stimulation of cerebellar fastigial nucleus inhibits amygdaloid kindling acquisition in Sprague-Dawley rats. Neurobiol Dis. 2008;29:52-8.

27. Paxinos G, Watson C. The Rat Brain in Stereotaxic Coordinates. Fourth ed. New York: Academic Press; 1998.

28. Racine RJ. Modification of seizure activity by electrical stimulation. II. Motor seizure. Electroencephalogr Clin Neurophysiol. 1972;32:281-94.

29. Tan SK, Janssen ML, Jahanshahi A, Chouliaras L, Visser-Vandewalle V, Lim LW, Steinbusch HW, Sharp T, Temel $Y$. High frequency stimulation of the subthalamic nucleus increases c-fos immunoreactivity in the dorsal raphe nucleus and afferent brain regions. J Psychiatr Res. 2011;45:1307-15.

30. Vlamings R, Visser-Vandewalle V, Kozan R, Kaplan S, Steinbusch HW, Temel Y. Bilateral high frequency stimulation of the subthalamic nucleus normalizes COX activity in the substantia nigra of Parkinsonian rats. Brain Res. 2009;1288:143-8.

31. Schlaug G, Antke C, Holthausen H, Arnold S, Ebner A, Tuxhorn I, Jäncke L, Lüders H, Witte OW, Seitz RJ. Ictal motor signs and interictal regional cerebral hypometabolism. Neurology. 1997;49:341-50.

32. Yune MJ, Lee JD, Ryu YH, Kim DI, Lee BI, Kim SJ. Ipsilateral thalamic hypoperfusion on interictal SPECT in temporal lobe epilepsy. J Nucl Med. 1998;39:281-5.

33. Martlé V, Peremans K, Audenaert K, Vermeire S, Bhatti S, Gielen I, Polis I, Van Ham L. Regional brain perfusion in epileptic dogs evaluated by technetium-99m-ethyl cysteinate dimer SPECT. Vet Radiol Ultrasound. 2009;50:655-9.

34. Loscher W, Ebert $U$, Wahnschaffe $U$, Rundfeldt $C$. Susceptibility of different cell layers of the anterior and posterior part of the piriform cortex to electrical stimulation and kindling: comparison with the basolateral amygdala and "area tempestas". Neuroscience. 1995;66: 265-76.

35. Weder BJ, Schindler K, Loher TJ, Wiest R, Wissmeyer M, Ritter P, Lovblad K, Donati F, Missimer J. Brain areas involved in medial temporal lobe seizures: a principal component analysis of ictal SPECT data. Hum Brain Mapp. 2006;27:520-34.

36. Schindler KA, Bialonski S, Horstmann MT, Elger CE, Lehnertz K. Evolving functional network properties and synchronizability during human epileptic seizures. Chaos. 2008;18:033119.

37. Nestler EJ. Review. Transcriptional mechanisms of addiction: role of DeltaFosB. Philos Trans R Soc Lond B Biol Sci. 2008;363:3245-55.

38. Voogd J. The human cerebellum. J Chem Neuroanat. 2003;26:243-52.

39. Duan XQ, Wu SL, Li T, Liang JC, Qiou JY, Rao ZR, Ju G. Expression and significance of three types of Fosimmunoreactive cells after gamma knife irradiation of the forebrain in the rat. Neurosci Res. 1999;33:99-104.

40. Dragunow M. Presence and induction of Fos B-like immunoreactivity in neural, but not non-neural, cells in adult rat brain. Brain Res. 1990;533:324-8.

41. Wikstrom M. Cytochrome c oxidase: 25 years of the elusive proton pump. Biochim Biophys Acta. 2004;1655:241-7.

42. Brzezinski $P$, Gennis RB. Cytochrome $c$ oxidase: exciting progress and remaining mysteries. J Bioenerg Biomembr. 2008;40:521-31.

43. Saelens X, Festjens N, Vande Walle L, van Gurp M, van Loo G, Vandenabeele P. Toxic proteins released from mitochondria in cell death. Oncogene. 2004;23:2861-74.

44. Niquet J, Liu H, Wasterlain CG. Programmed neuronal necrosis and status epilepticus. Epilepsia. 2005;46 Suppl 5:43-8.

45. Li T, Lu C, Xia Z, Xiao B, Luo Y. Inhibition of caspase-8 attenuates neuronal death induced by limbic seizures in a cytochrome c-dependent and Smac/DIABLO-independent way. Brain Res. 2006;1098: 204-11. 
46. Chuang YC. Mitochondrial dysfunction and oxidative stress in seizure-induced neuronal cell death. Acta Neurol Taiwan. 2010;19:3-15.

47. Zhao S, Aviles ER, Jr., Fujikawa DG. Nuclear translocation of mitochondrial cytochrome c, lysosomal cathepsins B and D, and three other death-promoting proteins within the first 60 minutes of generalized seizures. J Neurosci Res. 2010;88:1727-37.

48. Ju X, Mallet RT, Downey HF, Metzger DB, Jung ME. Intermittent hypoxia conditioning protects mitochondrial cytochrome $\mathrm{c}$ oxidase of rat cerebellum from ethanol withdrawal stress. J Appl Physiol. 2012;112:1706-14.

49. Heath RG, Harper JW. Ascending projections of the cerebellar fastigial nucleus to the hippocampus, amygdala, and other temporal lobe sites: evoked potential and histological studies in monkeys and cats. Exp Neurol. 1974;45:268-87.

50. Snider RS, Maiti A. Cerebellar contributions to the Papez circuit. J Neurosci Res. 1976;2: 133-46.

51. Berntson GG, Torello MW. Attenuation of septal hyperemotionality by cerebellar fastigial lesions in the rat. Physiol Behav. 1980;24:547-51.

52. Hermann BP, Bayless K, Hansen R, Parrish J, Seidenberg M. Cerebellar atrophy in temporal lobe epilepsy. Epilepsy Behav. 2005;7:279-87.

53. Li Y, Du H, Xie B, Wu N, Wang J, Wu G, Feng H, Jiang T. Cerebellum abnormalities in idiopathic generalized epilepsy with generalized tonic-clonic seizures revealed by diffusion tensor imaging. PLoS One. 2010;5:e15219.

54. Batini C, Compoint C, Buisseret-Delmas C, Daniel H, Guegan M. Cerebellar nuclei and the nucleocortical projections in the rat: retrograde tracing coupled to GABA and glutamate immunohistochemistry. J Comp Neurol. 1992;315:74-84.

55. Fisher R, Salanova V, Witt T, Worth R, Henry T, Gross R, Oommen K, Osorio I, Nazzaro J, Labar D, Kaplitt M, Sperling M, Sandok E, Neal J, Handforth A, Stern J, DeSalles A, Chung S, Shetter A, Bergen D, Bakay R, Henderson J, French J, Baltuch G, Rosenfeld W, Youkilis A, Marks W, Garcia P, Barbaro N, Fountain N, Bazil C, Goodman R, McKhann G, Babu Krishnamurthy K, Papavassiliou S, Epstein C, Pollard J, Tonder L, Grebin J, Coffey R, Graves N. Electrical stimulation of the anterior nucleus of thalamus for treatment of refractory epilepsy. Epilepsia. 2010;51:899-908.

56. Zhang Q, Wu ZC, Yu JT, Zhong XL, Xing YY, Tian Y, Miao D, Tan L. Anticonvulsant effect of unilateral anterior thalamic high frequency electrical stimulation on amygdala-kindled seizures in rat. Brain Res Bull. 2012;87:221-6.

57. Miller JW, Gray BC, Turner GM. Role of the fastigial nucleus in generalized seizures as demonstrated by GABA agonist microinjections. Epilepsia. 1993;34:973-8.

58. Min JK, Valentine PA, Teskey GC. Effect of complete and partial bilateral lesions of the deep cerebellar nuclei on amygdaloid kindling in rats. Epilepsia. 1998;39:692-9.

59. Maiti A, Snider RS. Cerebellar control of basal forebrain seizures: amygdala and hippocampus. Epilepsia. 1975;16:521-33. 


\section{Chapter 7}

General discussion and conclusion 
$102 \mid$ Chapter 7 


\section{General discussion and conclusion}

The role of the cerebellum in modulating behaviour is currently the subject of extensive investigation using a variety of techniques. Research aimed at the anatomical and functional connections of the cerebellum forms one way of addressing this topic. Deep brain stimulation (DBS) can affect distant structures and can thus be used to analyse the existence of functional connections between these structures. In chapter 1 we presented the results of a literature search showing that this method has not been used frequently to analyze functional connections with cerebellar structures. In our studies we analysed cerebellar activity following DBS-induced behaviour. More specifically, we presented findings on the changes of neuronal activity of individual deep cerebellar nuclei (DCbN) following DBS in animal models. Here we summarize the main findings of these studies, discuss their relevance, and propose future research.

\section{Cerebellum and behaviour}

The cerebellar cognitive affective syndrome (CCAS) and the cerebellar mutism syndrome (CMS, a.k.a. posterior fossa syndrome, PFS) described in patients with cerebellar lesions include inappropriate or disinhibited behaviour or decreased initiation of activity ${ }^{1,2}$. Studies on the relationship between impulsivity and the cerebellum are described in chapters $\mathbf{2}$ and $\mathbf{3}$. Here we demonstrated that DBS of the mediodorsal thalamic nucleus (MD) results in an increase of impulsive behaviour and decreased activity of the DCbN (chapter 2). In contrast, DBS of the ventrolateral thalamic nucleus (VL) had no influence on impulsivity and did not change neuronal activity of the DCbN. DBS of the subthalamic nucleus (STN) has previously been shown to improve motor parameters and decrease impulsivity in naïve rats ${ }^{3}$ as well as in a rat model of Parkinson's disease ${ }^{4}$. In chapter 3 we showed that DBS of this nucleus resulted in increased neuronal activity of the DCbN.

The dorsolateral periaqueductal grey (dIPAG) and the ventromedial hypothalamus (VMH) are both constituents of the fear network. DBS of these areas in rats leads to panic-like behaviour and can therefore be used as a model for panic attacks ${ }^{5,6}$. Using this model we showed that panic behaviour is accompanied by decreased activity of the DCbN (chapter 4). dIPAG stimulated animals that are returned to the experimental arena twelve hours after stimulation show a freezing response indicating conditioned fear $^{7}$. In contrast to the findings following acute dIPAG stimulation, we found no altered activity of DCbN following this conditioned response (chapter 5).

The relationship between the cerebellum and epilepsy was first suggested by Hunt when he noted the coexistence of myoclonic epilepsy with cerebellar atrophy ${ }^{8,9}$. Lesions of the cerebellum have been shown to induce or facilitate seizures and seizure spread. Cerebellar stimulation has been explored as possible treatment, with inconsistent results ${ }^{10}$. Amygdala kindling is a model in which repeated subthreshold 
stimulation of the amygdala causes seizures of increasing severity, which is used as a model of chronic epilepsy ${ }^{11}$. Fully kindled rats were characterized by a significantly decreased neuronal activity in the DCbN (chapter 6 ). This activity correlated negatively with the afterdischarge threshold.

These results lead us to conclude that abnormal or disruptive behaviour is accompanied by decreased activity in the $D C b N$, whereas favourable behaviour is associated with increased activity in the DCbN. These findings add to the evidence that the cerebellum plays a role in modulating behaviour. Several authors have suggested that the cerebellum in fact modulates all aspects of neurological functioning ${ }^{12,13}$. Schmahmann has suggested that cerebellar modulation serves as a dampener of oscillation around a homeostatic baseline ${ }^{13}$. Cerebellar damage then results in dysmetria of thought as well as of movement, depending on the localisation of the lesion. The exact manner in which the cerebellum does this is still unclear. It has become clear that the cerebellum is involved in motor learning processes by long term depression (LTD) mechanisms of Purkinje cells $\left(P C^{\prime} s\right)^{14,15}$. Functional imaging studies have shown that the cerebellum is activated in the process of motor sequence learning ${ }^{14}$. The cerebellar cortex shows activation in the early phase of learning, which shifts towards the $\mathrm{DCbN}$ during the learning process and finally disappears with extended practice ${ }^{14}$. This may be the explanation for the lack of activation changes found in the $D C b N$ in conditioned fear (chapter 5). It has been suggested that the cerebellum uses this learning process to form internal models, which are fine-tuned by error signals ${ }^{16,17}$. Using these internal models the brain can direct movements precisely and swiftly without the need to wait for sensory feedback ${ }^{18}$. It is suggested that the cerebellum forms internal models of mental processes in the same manner ${ }^{18}$.

\section{Cerebellar connections}

The uniform microstructure of the cerebellar cortex and the fact that it contains about 50 billion neurons suggests that the cerebellum has powerful mechanisms for information processing. It is likely that all incoming information is processed in the same manner, as mentioned previously. To be able to influence cognitive and behavioural processes the cerebellum needs to have afferent and efferent connections with cerebral areas that are involved in these processes. The input to the cerebellum has been shown to come from diffuse cortical areas, including the prefrontal and parietal cortex. In contrast to what was previously believed the output of the deep cerebellar nuclei is not solely directed at the primary motor cortex. Efferents from the $\mathrm{DCbN}$ project to diffuse areas in the cortex through the thalamus. Efferents from the $D C b N$ to the $V L$ thalamic nucleus are well-known, while efferents to the MD nucleus of the thalamus have been shown to exist more recently ${ }^{19}$. It has been shown that these nuclei form part of anatomical closed loops between the cerebellum and the cerebral cortex $^{20}$. Each of these loops seems to subserve a different function. The cerebellar 
recipient parts of the $M D$ and $V L$ nuclei of the thalamus project to the prefrontal cortex (PFC) and these cortical areas project back to the cerebellum, forming a loop that is thought to be involved in cognitive functioning. Our results support the existence of a cerebello-thalamo-cortical loop through the MD nucleus of the thalamus and the PFC and suggest that it is functionally involved in impulsive behaviour (chapter 2). In addition to closed loops with diverse cortical areas, recent tracing experiments in nonhuman primates have shown the existence of a closed loop between the cerebellum and the basal ganglia which seems to be independent of cortical input ${ }^{21}$. This loop is formed by a bisynaptic projection from the DN to the striatum ${ }^{22}$ and a similar projection from the STN to the cerebellar cortex via the pontine nuclei ${ }^{23}$. We have shown that there is indeed a functional connection between the STN and the DCbN (chapter 3). The projections from the STN to the cerebellum arise in all subterritories of the STN, suggesting that the pathways between the basal ganglia and the cerebellum are involved in motor as well as non-motor processing.

The cerebellum also has connections with multiple brain stem nuclei and midbrain structures. In the rat, projections from the DCbN to the PAG have been shown to exist ${ }^{24}$. Reciprocal connections between the cerebellum and widespread areas of the hypothalamus have been described using tracing experiments ${ }^{25}$. These anatomical connections linking the cerebellum with the fear network are supported by our results (chapter 4). Connections between the fastigial nucleus (FN) and the amygdala have been suggested based on behavioural changes following cerebellar lesioning or stimulation ${ }^{26,27}$. Classic electrophysiological studies also support a functional connection between the FN and the amygdala ${ }^{28}$, however, an exact anatomical pathway has never been shown ${ }^{29}$. The decreased metabolic activity in the DCbN following amygdala kindling which we found in chapter 6 may be the result of antidromic effects of stimulation in the basolateral amygdala. However, the rats in this model suffered from many stimulation-induced generalized seizures. Chronic epilepsy has been associated with cerebellar changes in human imaging studies, possibly related to duration and severity of the disorder ${ }^{30-33}$. Therefore the altered activity we find in the $\mathrm{DCbN}$ may also be explained as secondary to repeated seizure activity rather than a direct effect of electrical stimulation.

All cerebellar connections described above are based on anatomical and functional studies in animal models. Reproduction of these anatomical connections in the human situation has been difficult. However, with the advent of functional imaging the possibility has risen to study functional connections in the human brain in healthy controls as well as in neurological patients. Functional imaging such as PET, SPECT and fMRI is increasingly used in patients undergoing DBS to elucidate the mechanism of DBS. In chapter 1 we have summarized the functional imaging studies in patients with Parkinson's disease (PD) treated with DBS. Changes of activation are often seen in the cerebellum, suggesting functional connections between the stimulated structures and the cerebellum. Functional neuroimaging studies have also shown cerebellar activation during a variety of cognitive tasks ${ }^{34}$. Functional connectivity between the cerebellum 
and prefrontal and posterior parietal cortex was shown using resting-state functional connectivity $\mathrm{MRI}^{35}$, suggesting an anatomical connection. Recent developments in diffusion tensor imaging (DTI) have made it possible to analyse the connections of white matter tracts of the human cerebellum. Using this technique the connection between the dentate nucleus (DN) and the prefrontal and posterior parietal cortex described previously in monkeys has been confirmed in humans ${ }^{36}$. A connection between the STN and the cerebellum was also shown using DTI ${ }^{37}$.

The exact localization of activation changes in functional imaging of the cerebellum and especially the $\mathrm{DCbN}$ is however hampered by the small size and the lack of a reliable MRI atlas ${ }^{38}$. Recent improvement of neuroimaging techniques has resulted in an increased spatial resolution and now allows visualization of DCbN and cerebello-cortical sublayers. It has even been proven possible to visualize subunits of the $\mathrm{DN}^{38}$. A meta-analysis of functional imaging studies and human cerebellar lesion studies shows that there is a functional topography in the cerebellum in which sensorimotor functions are localized to lobules I-VI in the anterior lobe, extending into lobule VI and posterior lobules VIIIA and VIIIB. Cognitive functions are localized to the lateral regions of lobules VI and VII of the posterior lobe ${ }^{34}$. Functional connectivity studies support the mainly contralateral projection from cerebellum to cerebral cortex. Functional imaging during cognitive tasks show lateralization of language and spatial processing respectively to the right and left cerebellar hemispheres, which is in concordance with symptoms found in patients with lateralized cerebellar lesions ${ }^{34}$. When the analysis is directed to the $D C b N$, the $D N$, and especially the ventrocaudal region is activated in cognitive tasks. This is in concordance with anatomical projections to the prefrontal and posterior parietal cortices which originate mainly in the DN. In contrast, we found activation changes in all DCbN following behavioural changes induced by DBS. However, functional imaging of the interposite nucleus (IN) and the fastigial nucleus (FN) is still subject to methodological restrictions due to their limited volume. Further improvements of these imaging techniques are therefore necessary to be able to give reliable information on the activation of these $D C b N$ on functional imaging. Future research using these imaging techniques may enable us to further analyse anatomical and functional connections between the cerebellum and the rest of the brain and within the cerebellum itself. This approach has recently proven its value, for imaging of connections and activation patterns in neuropsychiatric diseases have given insight in the involvement of the cerebellum (for example ${ }^{39,40}$ ). It is to be expected that this knowledge will extend to other neurological and psychiatric disorders.

\section{Cerebellar stimulation}

Similar to DBS of cerebral targets, cerebellar stimulation has frequently been used in research settings as well as in clinical settings. Stimulation of cerebellar cortex or DCbN 
has been performed to analyse connections of the cerebellum with the limbic system $^{28,41}$ and to elucidate the role of the cerebellum in epilepsy ${ }^{42,43}$. Cerebellar stimulation as a treatment option has been studied intensively in relation to epilepsy (for review see Fountas et $a .^{10}{ }^{10}$. The rationale was based on the concept of an inhibitory influence of the cerebellum on the thalamus, resulting in decreased activity of excitatory thalamocortical projections. This would then result in decreased cortical excitability, thereby inhibiting seizures or seizure spread. However, these studies showed inconsistent results, possibly due to variations in methodology, such as targets and stimulation parameters. Furthermore, clinical studies included patients with different types of epilepsy and seizures. Interestingly, the patients included in these studies often showed improvement of cognitive functioning, either subjectively or psychologically assessed, which is independent of the effect on seizures ${ }^{10}$. Cerebellar stimulation has also been studied as a possible treatment option for spasticity especially in cerebral palsy (CP), with positive effects ${ }^{44}$. Most of the above mentioned studies applied cerebellar cortex stimulation. Only three animal studies performed stimulation of the DCbN. Two of these studies found an inhibition of seizure activity ${ }^{45,46}$. One study observed no effect on seizures induced by electrical stimulation, but found a significant reduction of spasticity ${ }^{47}$. In humans Sramka et al. performed bilateral stimulation of the DN leading to a transient improvement of seizure in all three patients ${ }^{48}$. Dentate nucleus stimulation was shown to improve spasticity and speech quality in a patient with spasticity due to $\mathrm{CP}^{49}$. This patient also showed improved status on psychological testing.

Cerebellar stimulation as a therapeutic option needs more research before it can be added to the standard treatment options in epilepsy and spasticity. With the recently improved stimulation hardware and software, it seems logical to go back to animal research to evaluate the effect of cerebellar stimulation and optimize target and stimulation settings. We have shown that generalized seizures elicited by amygdala kindling are associated with decreased activity in the DCbN (chapter 6). Activating the $\mathrm{DCbN}$ by low frequent stimulation may therefore prove beneficial. Since the FN seems to be the most important of the DCbN in epilepsy, it may be the best target to analyse.

With respect to behaviour, several options for further research present themselves. In the past cerebellar stimulation has been performed eliciting behavioural changes such as sham rage, predatory attack, grooming and eating ${ }^{50,51}$. It would be interesting to repeat and elaborate on these experiments using modern stimulation technology in animal models. A possible setup would be to evaluate the effect of stimulation of the different $\mathrm{DCbN}$ with high and low frequency on behavioural aspects such as impulsivity, fear and depression. Further experiments might involve similar stimulation studies in animal models of neuropsychiatric diseases. The cerebellum has been implicated in diseases such as schizophrenia, autism and depression. It was suggested that these patients suffer from a lack of specific internal models related to mental processes ${ }^{18}$. Cerebellar stimulation may prove beneficial by improving the 
function of the cerebellum leading to better internal model to direct behaviour. Results from cerebellar stimulation in animal models of these diseases may in the end lead to therapeutic implementation.

\section{Conclusion}

This thesis demonstrates that the cerebellum plays a role in the modulation of behaviour. Decreased activity of the deep cerebellar nuclei is associated with abnormal behaviour (impulsivity, panic attacks and seizures). This adds to the body of evidence that the cerebellum modulates all aspects of neurological functioning, possibly by forming internal models that allow motor and mental processes to be performed accurately and swiftly. Cerebellar lesions can lead to behavioural and cognitive problems, depending on the localisation of the lesion. This also explains cognitive and behavioural problems seen in congenital cerebellar abnormalities and in children following cerebellar tumour resection. Furthermore cerebellar abnormalities are found in neuropsychiatric diseases. These aspects of cerebellar function still receive too little attention in clinical practice. Future studies are necessary to increase our knowledge on the exact role of the cerebellum and the mechanisms underlying its influence on the brain. This will hopefully lead to more awareness of the presence of cognitive and behavioural problems in patients with cerebellar lesions. Finally, our results justify the evaluation of direct cerebellar stimulation in models of neurological and psychiatric disease. 


\section{References}

1. Schmahmann JD, Sherman JC. The cerebellar cognitive affective syndrome. Brain. 1998; 121(Pt 4): 561-79.

2. Wells EM, Walsh KS, Khademian ZP, Keating RF, Packer RJ. The cerebellar mutism syndrome and its relation to cerebellar cognitive function and the cerebellar cognitive affective disorder. Dev Disabil Res Rev. 2008;14:221-8.

3. Desbonnet L, Temel Y, Visser-Vandewalle V, Blokland A, Hornikx V, Steinbusch HW. Premature responding following bilateral stimulation of the rat subthalamic nucleus is amplitude and frequency dependent. Brain Res. 2004;1008:198-204.

4. Temel Y, Visser-Vandewalle V, Aendekerk B, Rutten B, Tan S, Scholtissen B, Schmitz C, Blokland A, Steinbusch HW. Acute and separate modulation of motor and cognitive performance in parkinsonian rats by bilateral stimulation of the subthalamic nucleus. Exp Neurol. 2005;193:43-52.

5. Lim LW, Blokland A, Visser-Vandewalle V, Vlamings R, Sesia T, Steinbusch H, Schruers K, Griez E, Temel $Y$. High-frequency stimulation of the dorsolateral periaqueductal gray and ventromedial hypothalamus fails to inhibit panic-like behaviour. Behav Brain Res. 2008; 193:197-203.

6. Schenberg LC, Bittencourt AS, Sudre EC, Vargas LC. Modeling panic attacks. Neurosci Biobehav Rev. 2001;25:647-59.

7. Lim SY, Lang AE. The nonmotor symptoms of Parkinson's disease--an overview. Mov Disord. 2010;25 Suppl 1:S123-30.

8. Hunt JR. Dyssynergia cerebellaris progressiva - a chronic progressive form of cerebellar tremor. Brain. 1914;37:247-68.

9. Dow RS, Fernandez-Guardiola A, Manni E. The influence of the cerebellum on experimental epilepsy. Electroencephalogr Clin Neurophysiol. 1962;14:383-98.

10. Fountas KN, Kapsalaki E, Hadjigeorgiou G. Cerebellar stimulation in the management of medically intractable epilepsy: a systematic and critical review. Neurosurg Focus. 2010;29:E8.

11. Racine RJ. Modification of seizure activity by electrical stimulation. II. Motor seizure. Electroencephalogr Clin Neurophysiol. 1972;32:281-94.

12. Leiner HC, Leiner AL, Dow RS. Does the cerebellum contribute to mental skills? Behav Neurosci. 1986;100:443-54.

13. Schmahmann JD. Disorders of the cerebellum: ataxia, dysmetria of thought, and the cerebellar cognitive affective syndrome. J Neuropsychiatry Clin Neurosci. 2004;16:367-78.

14. Doyon J, Song AW, Karni A, Lalonde F, Adams MM, Ungerleider LG. Experience-dependent changes in cerebellar contributions to motor sequence learning. Proc Natl Acad Sci U S A. 2002;99:1017-22.

15. Sacchetti B, Scelfo B, Strata P. The cerebellum: synaptic changes and fear conditioning. Neuroscientist. 2005;11:217-27.

16. Wolpert DM, Miall RC, Kawato M. Internal models in the cerebellum. Trends in cognitive sciences. 1998;2:338-47.

17. Bastian AJ. Learning to predict the future: the cerebellum adapts feedforward movement control. Curr Opin Neurobiol. 2006;16:645-9.

18. Ito M. Control of mental activities by internal models in the cerebellum. Nat Rev Neurosci. 2008;9: 304-13.

19. Yamamoto $\mathrm{T}$, Yoshida K, Yoshikawa H, Kishimoto $\mathrm{Y}, \mathrm{Oka} \mathrm{H}$. The medial dorsal nucleus is one of the thalamic relays of the cerebellocerebral responses to the frontal association cortex in the monkey: horseradish peroxidase and fluorescent dye double staining study. Brain Res. 1992;579:315-20.

20. Kelly RM, Strick PL. Cerebellar loops with motor cortex and prefrontal cortex of a nonhuman primate. J Neurosci. 2003;23:8432-44

21. Bostan AC, Strick PL. The cerebellum and basal ganglia are interconnected. Neuropsychol Rev. 2010;20:261-70.

22. Hoshi E, Tremblay L, Feger J, Carras PL, Strick PL. The cerebellum communicates with the basal ganglia. Nat Neurosci. 2005;8:1491-3.

23. Bostan AC, Dum RP, Strick PL. The basal ganglia communicate with the cerebellum. Proc Natl Acad Sci U S A. 2010;107:8452-6. 
24. Teune TM, van der Burg J, van der Moer J, Voogd J, Ruigrok TJ. Topography of cerebellar nuclear projections to the brain stem in the rat. Prog Brain Res. 2000;124:141-72.

25. Haines DE, Dietrichs E, Mihailoff GA, McDonald EF. The cerebellar-hypothalamic axis: basic circuits and clinical observations. Int Rev Neurobiol. 1997;41:83-107.

26. Snider RS, Maiti A. Cerebellar contributions to the Papez circuit. J Neurosci Res. 1976;2:133-46.

27. Berntson GG, Torello MW. Attenuation of septal hyperemotionality by cerebellar fastigial lesions in the rat. Physiol Behav. 1980;24:547-51.

28. Heath RG, Harper JW. Ascending projections of the cerebellar fastigial nucleus to the hippocampus, amygdala, and other temporal lobe sites: evoked potential and histological studies in monkeys and cats. Exp Neurol. 1974;45:268-87.

29. Strick PL, Dum RP, Fiez JA. Cerebellum and nonmotor function. Annu Rev Neurosci. 2009;32:413-34.

30. Hermann BP, Bayless K, Hansen R, Parrish J, Seidenberg M. Cerebellar atrophy in temporal lobe epilepsy. Epilepsy Behav. 2005;7:279-87.

31. Sandok EK, O'Brien TJ, Jack CR, So EL. Significance of cerebellar atrophy in intractable temporal lobe epilepsy: a quantitative MRI study. Epilepsia. 2000;41:1315-20.

32. Leifer D, Cole DG, Kowall NW. Neuropathologic asymmetries in the brain of a patient with a unilateral status epilepticus. J Neurol Sci. 1991;103:127-35.

33. Hagemann G, Lemieux L, Free SL, Krakow K, Everitt AD, Kendall BE, Stevens JM, Shorvon SD. Cerebellar volumes in newly diagnosed and chronic epilepsy. J Neurol. 2002;249:1651-8.

34. Stoodley CJ. The cerebellum and cognition: evidence from functional imaging studies. Cerebellum. 2012;11:352-65.

35. Allen G, McColl R, Barnard H, Ringe WK, Fleckenstein J, Cullum CM. Magnetic resonance imaging of cerebellar-prefrontal and cerebellar-parietal functional connectivity. Neuroimage. 2005;28:39-48.

36. Jissendi P, Baudry S, Baleriaux D. Diffusion tensor imaging (DTI) and tractography of the cerebellar projections to prefrontal and posterior parietal cortices: a study at 3T. J Neuroradiol. 2008;35:42-50.

37. Aravamuthan BR, Muthusamy KA, Stein JF, Aziz TZ, Johansen-Berg H. Topography of cortical and subcortical connections of the human pedunculopontine and subthalamic nuclei. Neuroimage. 2007;37:694-705.

38. Kuper $M$, Thurling $M$, Maderwald $S$, Ladd ME, Timmann D. Structural and functional magnetic resonance imaging of the human cerebellar nuclei. Cerebellum. 2012;11:314-24.

39. Li Y, Du H, Xie B, Wu N, Wang J, Wu G, Feng H, Jiang T. Cerebellum abnormalities in idiopathic generalized epilepsy with generalized tonic-clonic seizures revealed by diffusion tensor imaging. PLoS One. 2010;5:e15219.

40. Catani M, Jones DK, Daly E, Embiricos N, Deeley Q, Pugliese L, Curran S, Robertson D, Murphy DG. Altered cerebellar feedback projections in Asperger syndrome. Neuroimage. 2008;41:1184-91.

41. Anand BK, Malhotra CL, Singh B, Dua S. Cerebellar projections to limbic system. J Neurophysiol. 1959;22:451-7.

42. Wang S, Wu DC, Ding MP, Li Q, Zhuge ZB, Zhang SH, Chen Z. Low-frequency stimulation of cerebellar fastigial nucleus inhibits amygdaloid kindling acquisition in Sprague-Dawley rats. Neurobiol Dis. 2008;29:52-8.

43. Rubio C, Custodio V, Juarez F, Paz C. Stimulation of the superior cerebellar peduncle during the development of amygdaloid kindling in rats. Brain Res. 2004;1010:151-5.

44. Davis R. Cerebellar stimulation for cerebral palsy spasticity, function, and seizures. Arch Med Res. 2000;31:290-9.

45. Babb TL, Mitchell AG, Jr., Crandall PH. Fastigiobulbar and dentatothalamic influences on hippocampal cobalt epilepsy in the cat. Electroencephalogr Clin Neurophysiol. 1974;36:141-54.

46. Hutton JT, Frost JD, Jr., Foster J. the influence of the cerebellum in cat penicillin epilepsy. Epilepsia. 1972;13:401-8.

47. Hemmy DC, Larson SJ, Sances A, Jr., Millar EA. The effect of cerebellar stimulation on focal seizure activity and spasticity in monkeys. J Neurosurg. 1977;46:648-53.

48. Sramka M, Fritz G, Galanda M, Nadvornik P. Some observations in treatment stimulation of epilepsy. Acta Neurochir (Wien). 1976(23 Suppl):257-62.

49. Schvarcz JR, Sica RE, Morita E. Chronic self-stimulation of the dentate nucleus for the relief of spasticity. Acta Neurochir Suppl (Wien). 1980;30:351-9. 
50. Reis DJ, Doba N, Nathan MA. Predatory attack, grooming, and consummatory behaviors evoked by electrical stimulation of cat cerebellar nuclei. Science. 1973;182:845-7.

51. Zanchetti A, Zoccolini A. Autonomic hypothalamic outbursts elicited by cerebellar stimulation. J Neurophysiol. 1954;17:475-83. 

Summary 
114 


\section{Summary}

The role of the cerebellum in modulating behaviour is currently a subject of extensive investigation using a variety of techniques. Research aimed at the anatomical and functional connections of the cerebellum forms one way of addressing this topic. Deep brain stimulation (DBS) can affect distant structures and can thus be used to analyse the existence of functional connections with cerebellar structures. In our study we analysed the involvement of the cerebellum in behaviour modulated by DBS. In this thesis, the results of several studies in which we examined the neuronal activity of the deep cerebellar nuclei (DCbN) following DBS in animal models have been presented.

Changes of impulsive behaviour have been described in patients with cerebellar lesions. In chapters $\mathbf{2}$ and $\mathbf{3}$ we studied the relationship between impulsivity and the cerebellum. Here we demonstrated that DBS of the mediodorsal nucleus of the thalamus (MD) results in an increase of impulsive behaviour and decreased activity of the DCbN (chapter 2). In contrast, DBS of the ventrolateral thalamic nucleus (VL) had no influence on impulsivity and did not change neuronal activity of the DCbN. DBS of the subthalamic nucleus (STN) has previously been shown to improve motor parameters and decrease impulsivity in naïve rats as well as in a rat model of Parkinson's disease. In chapter $\mathbf{3}$ we showed that DBS of this nucleus resulted in increased neuronal activity of the DCbN.

The dorsolateral periaqueductal grey (dIPAG) and the ventromedial hypothalamus (VMH) are both constituents of the fear network. DBS of these areas in rats leads to panic-like behaviour and can therefore be used as a model for panic attacks. Using this model we showed that panic behaviour is accompanied by decreased activity of the DCbN (chapter 4). dIPAG stimulated animals that are returned to the experimental arena twelve hours after stimulation show a freezing response indicating conditioned fear. In contrast to the findings following acute dIPAG stimulation, we found no altered activity of $\mathrm{DCbN}$ following this conditioned response (chapter $\mathbf{5}$ ).

The relationship between the cerebellum and epilepsy was first suggested by Hunt when he noted the coexistence of myoclonic epilepsy with cerebellar atrophy. Lesions of the cerebellum have been shown to induce or facilitate seizures and seizure spread. Cerebellar stimulation has been explored as possible treatment, with inconsistent results. Amygdala kindling is a model in which repeated subthreshold stimulation of the amygdala causes seizures of increasing severity, which is used as a model of chronic epilepsy. Fully kindled rats were characterized by a significantly decreased neuronal activity in the DCbN (chapter 6 ). This activity correlated negatively with the afterdischarge threshold.

These results lead us to conclude that abnormal or disruptive behaviour is accompanied by decreased activity in the $\mathrm{DCbN}$, whereas favourable behaviour is associated with increased activity in the DCbN. This adds to the evidence that the cerebellum plays a role in modulating behaviour. It has been proposed that the cerebellum forms internal models of neurological processes which are constantly fine- 
tuned by error signals. These internal models allow the brain to direct these processes, either motor or mental, without having to rely on direct sensory feedback. Damage to the cerebellum may hamper the fine-tuning and accessibility of these models, leading to motor, behavioural or cognitive problems depending on the localisation of the lesion.

The uniform manner in which the cerebellum seems to process information means that the neurological processes it has influence on are defined by its anatomical connections. Animal research has shown that there are several independent loops between the cerebellum and the cortex, passing through several thalamic nuclei, as well as between the cerebellum and the basal ganglia. Our results confirm the existence of functional connections between the cerebellum and the MD nucleus of the thalamus (chapter 2), the STN (chapter 3), the dIPAG and the VMH (chapter 4), and the amygdala (chapter 6 ). Improvement of neuroimaging techniques such as functional MRI and diffusion tensor imaging have already proven their value in analyzing these anatomical and functional connections in humans. Their role in this research is expected to grow in the coming years.

Cerebellar stimulation has been performed in the past in animal models to analyse connections of the cerebellum with the limbic system and to elucidate the role of the cerebellum in epilepsy. It has also been studied as a therapeutic option in patients with epilepsy and spasticity, with inconsistent results. We suggest future research using DBS of the cerebellar nuclei to further evaluate their role in behaviour and epilepsy and to analyse the possible therapeutic effect in animal models of epilepsy and neuropsychiatric diseases. The results of these studies may lead to therapeutic implementation of cerebellar stimulation.

\section{Conclusion}

This thesis demonstrates that the cerebellum plays a role in the modulation of behaviour. Decreased activity of the deep cerebellar nuclei is associated with abnormal behaviour (impulsivity, panic attacks and seizures). This adds to the body of evidence that the cerebellum modulates all aspects of neurological functioning, possibly by forming internal models that allow motor and mental processes to be performed accurately and swiftly. Cerebellar lesions can lead to behavioural and cognitive problems, depending on the localisation of the lesion. This also explains cognitive and behavioural problems seen in congenital cerebellar abnormalities and in children following cerebellar tumour resection. Furthermore cerebellar abnormalities are found in neuropsychiatric diseases. These aspects of cerebellar function still receive too little attention in clinical practice. Future studies are necessary to increase our knowledge on the exact role of the cerebellum and the mechanisms underlying its influence on the brain. This will hopefully lead to more awareness of the presence of cognitive and behavioural problems in patients with cerebellar lesions. Finally, our results justify the 
evaluation of direct cerebellar stimulation in models of neurological and psychiatric disease. 

Samenvatting 
120 


\section{Samenvatting}

De rol van het cerebellum in het moduleren van gedrag wordt momenteel met verschillende technieken onderzocht. Een van deze technieken betreft het onderzoeken van anatomische en functionele verbindingen van het cerebellum. Diepe hersenstimulatie (DBS; deep brain stimulation) kan gebieden op afstand beïnvloeden en kan dus worden gebruikt om de aanwezigheid van functionele verbindingen van de gestimuleerde gebieden met de kleine hersenen te onderzoeken. In onze studie onderzochten wij de betrokkenheid van het cerebellum bij gedrag dat gemoduleerd was door DBS. In dit proefschrift hebben wij de resultaten gepresenteerd van een aantal studies waarin we de neuronale activiteit van de diepe kernen van het cerebellum (DCbN; deep cerebellar nuclei) als gevolg van DBS in diermodellen hebben onderzocht.

Veranderingen van impulsief gedrag zijn beschreven bij patiënten met schade van het cerebellum. In hoofdstuk $\mathbf{2}$ en $\mathbf{3}$ hebben wij het verband tussen het cerebellum en impulsiviteit onderzocht. We hebben aangetoond dat DBS van de mediodorsale (MD) thalamuskern leidt tot een toename van impulsiviteit en een afname van de neuronale activiteit in de DCbN (hoofdstuk 2). DBS van de ventrolaterale thalamuskern (VL) heeft daarentegen geen invloed op impulsiviteit of neuronale activiteit van de DCbN. Het is al eerder aangetoond dat DBS van de subthalamische kern (STN) in naïeve ratten en in een ratmodel van de ziekte van Parkinson leidt tot een verbetering van motore parameters en een afname van impulsiviteit. In hoofdstuk 3 hebben we laten zien dat DBS van deze kern leidt tot een toename van de neuronale activiteit in de DCbN.

Het dorsolaterale periaqueductale grijs (dIPAG) en de ventromediale hypothalamus (VMH) maken beide onderdeel uit van het angst netwerk. DBS van deze gebieden leidt tot paniekachtig gedrag en kan worden gebruikt als een model voor paniekaanvallen. Gebruik makend van dit model hebben wij aangetoond dat paniekgedrag gepaard gaat met een afgenomen neuronale activiteit in de $\mathrm{DCbN}$ (hoofdstuk 4). Dieren die in het dIPAG zijn gestimuleerd en 12 uur later weer terug worden gezet in de experimentele arena vertonen angstgedrag hetgeen duidt op geconditioneerde angst. In tegenstelling tot de bevindingen na acute dIPAG-stimulatie vonden we geen verandering in de neuronale activiteit van de $\mathrm{DCbN}$ na dergelijke geconditioneerde angst (hoofdstuk 5).

De relatie tussen epilepsie en het cerebellum werd voor het eerst gelegd door Hunt toen hij het samen voorkomen van myoclone epilepsie en cerebellaire atrofie beschreef. Letsels van het cerebellum kunnen leiden tot epileptische aanvallen of het opwekken en spreiden hiervan faciliteren. Cerebellaire stimulatie werd reeds onderzocht als mogelijke behandeling, met tegenstrijdige resultaten. Amygdala kindling is een model waarbij herhaalde elektrische stimulatie van de amygdala leidt tot epileptische aanvallen met toenemende ernst, hetgeen gebruikt wordt als model voor chronische epilepsie. Volledig gekindelde ratten toonden een significante afname 
van de neuronale activiteit in de $\mathrm{DCbN}$ (hoofdstuk 6). Deze activiteit toonde een negatieve correlatie met de afterdischarge drempel.

Deze resultaten hebben geleid tot de conclusie dat afwijkend gedrag is geassocieerd met een afgenomen activiteit van de $\mathrm{DCbN}$ terwijl gunstig gedrag is geassocieerd met een toename van deze activiteit. Dit versterkt het bewijs dat het cerebellum is betrokken bij het moduleren van gedrag. $\mathrm{Er}$ is al voorgesteld dat het cerebellum interne modellen vormt van neurologische processen en dat deze modellen continu worden bijgeschaafd door feedback signalen. Deze interne modellen stellen de hersenen in staat deze motore en mentale processen vlot te laten verlopen zonder afhankelijk te zijn van directe feedback. Beschadiging van het cerebellum kan het bijwerken en de beschikbaarheid van deze modellen hinderen en leiden tot motorische, gedragsmatige of cognitieve problemen, afhankelijk van welk gebied van het cerebellum is aangedaan.

De uniforme manier waarop het cerebellum informatie lijkt te verwerken betekent dat de neurologische processen waar het invloed op uitoefent bepaald worden door de anatomische verbindingen. Dierexperimenteel onderzoek heeft aangetoond dat er meerdere onafhankelijke gesloten circuits bestaan tussen de cerebrale cortex en het cerebellum die door meerdere thalamuskernen lopen. Tevens bestaat er een dergelijk circuit tussen de basale kernen en het cerebellum. Onze resultaten bevestigen de aanwezigheid van functionele verbindingen tussen het cerebellum en de MD thalamuskern (hoofdstuk 2), STN (hoofdstuk 3), het dIPAG en de $\mathrm{VMH}$ (hoofdstuk 4) en de amygdala (hoofdstuk 6). Verbetering van beeldvormende technieken zoals functionele MRI en diffusion tensor imaging zijn al waardevol gebleken in het onderzoek van deze verbindingen in mensen. Hun rol in dit onderzoek zal naar verwachting alleen maar toenemen in de komende jaren.

Cerebellaire stimulatie werd in het verleden reeds toegepast om verbindingen van het cerebellum met het limbische systeem te onderzoeken en om de rol van het cerebellum in epilepsie op te helderen. Het werd tevens onderzocht als mogelijke therapeutische optie in patiënten met epilepsie en spasticiteit, met wisselend resultaat. Wij stellen verder onderzoek voor met DBS van de DCbN om hun rol in gedrag en epilepsie verder te verduidelijken en om het mogelijk therapeutische effect te onderzoeken in diermodellen van epilepsie en neuropsychiatrische ziektebeelden. De resultaten van deze studies zouden kunnen leiden tot de therapeutische implementatie van cerebellaire stimulatie.

\section{Conclusie}

Dit proefschrift toont aan dat het cerebellum een rol speelt in het moduleren van gedrag. Afgenomen activiteit in de diepe cerebellaire kernen is geassocieerd met afwijkend gedrag (impulsiviteit, angst en epilepsie). Dit voegt toe aan het bewijs dat het cerebellum alle neurologische processen beïnvloedt, mogelijk door interne 
modellen te vormen die snelle en accurate uitvoering van motore en mentale processen mogelijk maken. Cerebellaire laesies kunnen leiden tot motore en gedragsproblemen, afhankelijk van de lokalisatie van de laesie. Dit verklaart ook de cognitieve en gedragsproblemen die worden gezien in kinderen met aangeboren afwijkingen van het cerebellum of na verwijdering van een cerebellaire tumor. Verder worden cerebellaire afwijkingen ook gevonden in neuropsychiatrische aandoeningen. Deze aspecten van de functie van het cerebellum krijgen nog veel te weinig aandacht in de klinische praktijk. Verder onderzoek is noodzakelijk om de precieze rol van het cerebellum en de manier waarop het de hersenen beïnvloedt te begrijpen. Dit leidt hopelijk tot meer aandacht voor de aanwezigheid van cognitieve en gedragsproblemen in patiënten met cerebellaire beschadigingen. Als laatste rechtvaardigen onze resultaten onderzoek naar directe cerebellaire stimulatie in modellen van neurologische en psychiatrische aandoeningen. 

List of publications 
126 


\section{List of publications}

Rijkers K, Majoie HJM, Aalbers MW, Philippens M, Doenni VM, Vles JSH, Steinbusch HWM, Moers-Hornikx VMP, Hopkins DA, Hoogland G. Rat vagus nerve stimulation model of seizure suppression: nNOS and DeltaFos B changes in the brainstem. J Chem Neuroanat. 2012 Dec;46(1-2):1-9.

Moers-Hornikx VMP, Vles JSH, Hemmes RJ, Lim LW, Hoogland G, Steinbusch HWM, Temel Y. c-Fos expression in the deep cerebellar nuclei in a rat model of conditioned fear. J Exp Clin Med. 2012;29:201-7.

Strackx E, Gantert M, Moers-Hornikx VMP, van Kooten IAJ, Rieke R, Hurter H, Lemmens MAM, Steinbusch HWM, Zimmermann LI, Vles JSH, Garnier Y, Gavilanes AWD, Kramer BW. Increased number of cerebellar granule cells and astrocytes in the internal granule layer in sheep following prenatal intra-amniotic injection of lipopolysaccharide. Cerebellum. 2012 Mar;11(1):132-44.

Moers-Hornikx VMP, Vles JSH, Tan SKH, Cox K, Hoogland G, Steinbusch HWM, Temel Y. Cerebellar nuclei are activated by high-frequency stimulation of the subthalamic nucleus. Neurosci Lett. 2011 Jun 1;496(2):111-5.

Moers-Hornikx VMP, Vles JSH, Lim LW, Ayyildiz M, Kaplan S, Gavilanes AWD, Hoogland G, Steinbusch HWM, Temel Y. Periaqueductal grey stimulation induced panic-like behaviour is accompanied by deactivation of the deep cerebellar nuclei. Cerebellum. 2011 Mar;10(1):61-9.

Braakman HMH, Moers-Hornikx VMP, Arts BMG, Hupperts RMM, Nicolai J. Pearls \& Oy-sters: electroconvulsive therapy in anti-NMDA receptor encephalitis. Neurology. 2010 Sep 7;75(10):e44-6.

Moers-Hornikx VMP, Sesia T, Basar K, Lim LW, Hoogland G, Steinbusch HW, Gavilanes AWD, Temel Y, Vles JSH. Cerebellar nuclei are involved in impulsive behaviour. Behav Brain Res. 2009 Nov 5;203(2):256-63.

Desbonnet L, Temel Y, Visser-Vandewalle V, Blokland A, Hornikx VMP, Steinbusch HWM. Premature responding following bilateral stimulation of the rat subthalamic nucleus is amplitude and frequency dependent. Brain Res. 2004 May 22;1008(2):198204. 

Dankwoord 
130 


\section{Dankwoord}

Het is eindelijk zover, mijn boekje is af! Het is een ontdekkingstocht geweest. Toen ik begon had ik geen idee van de hobbels die ik onderweg zou tegenkomen, en meermaals heb ik getwijfeld of ik de reis af zou maken. Zonder de praktische en mentale ondersteuning van heel veel mensen zou het me niet gelukt zijn. Daarom wil ik hier mijn dank uitspreken aan allen die (een stukje) met me meegereisd zijn.

Geachte Prof. dr. Vles, beste Hans. Zonder jou was dit boekje er nooit geweest. Toen je me vroeg of ik interesse had om samen met jou onderzoek te doen naar het cerebellum was ik vereerd. Het was een sprong in het diepe; we wisten toen nog niet of de resultaten tot een boekje zouden kunnen leiden. Gelukkig is het uiteindelijk toch gelukt. Je bewaakte de voortgang en fungeerde als stok achter de deur als ik moeite had mijn deadlines te halen. Je deur stond voor me open, ook al had ik dat niet altijd door. $\mathrm{Nu}$ is het dan toch gelukt. Jij hebt er altijd vertrouwen in gehad, daar ben ik je heel dankbaar voor. Bedankt dat je mijn promotor wilt zijn.

Geachte Prof. dr. Temel, beste Yasin. Ik heb je leren kennen toen ik als student mijn wetenschapsstage bij je deed. Toen was ik al onder de indruk van de manier waarop jij zonder enige moeite aan de lopende band nieuwe onderzoeksideeën uit je mouw kon schudden. Via een kleine omweg ben ik voor mijn promotieonderzoek weer bij je terecht gekomen. Dankjewel dat ik van de expertise en het materiaal van jouw onderzoekgroep gebruik heb mogen maken. Jouw enthousiasme voor de wetenschap werkt aanstekelijk. Je leerde me de onderzoeksresultaten op een andere manier te bekijken, om tot hele nieuwe inzichten te komen. En iedere keer als ik twijfelde riep je "komt goed", en dat was ook zo. Ik ben heel blij dat je mijn promotor wilt zijn.

Beste Govert, als kamergenootje van Yasin was je de klos om als dagelijks begeleider op te treden in het lab. Ondanks (of misschien wel dankzij) dat het je vakgebied niet helemaal was heb je heel veel bijgedragen aan het onderzoek. Je verraste me op zijn tijd door een kritische vraag te stellen. Mede dankzij jouw steun hebben we uiteindelijk de data van de cerebelli van gekindelde ratten toch kunnen toevoegen. Daarnaast wil ik je danken voor een luisterend oor. Fijn dat je mijn copromotor wilt zijn.

Prof. dr. Van Oostenbrugge, beste Robert, voorzitter van mijn beoordelingscommissie en opleider. Ik wil je bedanken voor je kritische blik bij het beoordelen van het manuscript, maar ook voor de ruimte die ik kreeg om tijdens mijn opleiding aan dit proefschrift te werken. Daarnaast wil ik je bedanken voor de opleiding die ik krijg en voor je vertrouwen in mijn neurologisch kunnen.. De overige leden van de beoordelingscommissie prof. dr. A. Benazzouz, dr. C. Catsman-Berrevoets, prof. dr. J. 
van Overbeeke en prof. dr. R.J. Stokroos wil ik bedanken voor hun beoordeling van het manuscript.

Collega's van het lab, ik heb een jaar bij jullie mogen vertoeven en ik heb het er goed naar mijn zin gehad. Vooral mijn kamergenootjes Kim, Ivona, Evi, Frank en Lucas wil ik danken voor jullie positieve energie. Verder Rinske, Thibaut, Anthony en Sonny voor jullie hulp bij praktische zaken en uiteraard voor het ter beschikking stellen van jullie cerebelli en bijdragen aan mijn artikelen. Hellen en Marjan, dank voor jullie hulp en expertise in het lab. Prof. dr. Steinbusch, dank voor het ter beschikking stellen van de faciliteiten.

Kimberly en Rob, "mijn" studenten, dank dat jullie mij hebben willen helpen met de praktische uitvoering van mijn experimenten en analyses, jullie hebben voor mij vele uren achter snijmachines en microscopen doorgebracht. Daarnaast hebben jullie ook een mooie bijdrage geleverd aan het uitwerken van de resultaten. Soms waren jullie nog kritischer dan ik en dat heeft het resultaat alleen maar goed gedaan.

Desiree, je kwam pas later in het traject in beeld maar je kennis van de gang van zaken rondom een promotie is een grote steun. Tiny, dank voor het maken van de mooie layout. Het is leuk om te zien hoeveel plezier jij er in hebt, terwijl het voor mij waarschijnlijk alleen maar frustratie was geweest.

(Oud)-assistenten van de neurologie, van degenen die samen met mij zijn begonnen heb ik inmiddels wel de grootste vertraging opgelopen. Anderen zijn later gekomen en hebben mij (bijna) ingehaald. Maar allemaal toonden jullie interesse in het verloop van het onderzoek en hebben jullie mij aangemoedigd om de reis af te maken. Mede dankzij deze aanmoedigingen is het dan nu een feit. Pim, dankjewel voor je opstapje naar het idee voor mijn kaft. Ik wens je veel succes met het ontwerpen van die van jou.

Lieve Brigitte, we hebben elkaar pas goed leren kennen op de KNF; is het toch nog ergens goed voor geweest... Daar is het gelukkig niet bij gebleven. Je mentale steun en soms onorthodoxe adviezen hebben zeker bijgedragen aan het afronden van dit boekje... Je relativeringsvermogen heeft mij door meerdere moeilijke momenten heen gesleept. Je bent een lieve meid en een fijne vriendin. Ik wens je heel veel succes met je Antilliaanse ambities en ik kom zeker een keer op (werk)bezoek! Dankjewel dat je mijn paranimf wilt zijn.

Kim, samen met jou op een kamer in het lab voelde ik me al meteen een beetje thuis. Zowel over onderzoek als over moederschap en alles wat daarbij komt kijken konden we het goed vinden. Daarbij hebben we dankzij jouw inbreng het kindling stuk toch in dit boekje kunnen opnemen. Ik heb dankbaar gebruik gemaakt van jouw 
ervaringen met betrekking tot het promoveren. Dankjewel dat ik bij jou mocht uithuilen als het tegenzat. Ik ben heel blij dat je mijn paranimf wilt zijn.

Vader en moeder, jullie waren er gewoon altijd, ook al had ik dat niet altijd door. Jullie hebben me geholpen de mens te worden die ik nu ben. Moeder, dankjewel voor alle trapgesprekken. Ik hoop dat je nu trots bent.

Pap en Mam, jullie bijdrage aan dit proefschrift zit vooral in de ondersteunende logistiek. Zonder jullie hulp was de combinatie van kinderen en promoveren niet mogelijk geweest. Mam bedankt voor je hulp met mijn kaft.

Esther, Jeroen en Vincent, we zijn samen opgegroeid en ieder onze eigen weg gegaan. Als we elkaar zien is het altijd gezellig, al is het helaas niet zo vaak. Misschien dat het er nu wat vaker van komt. Marcel en Petry, jullie vullen de familie perfect aan. Yvonne en Bart, ik geniet iedere keer van de wervelwind die binnenkomt. Jullie kijk op het leven kan erg verfrissend zijn.

Myrthe, Rianne, Xander. Jullie maken mijn leven compleet. Jullie onvoorwaardelijke liefde en stralende lach maakt alles goed. Dankjewel dat jullie mijn kaft zo mooi hebben versierd! Kus en knuffel.

Lieve Rob. Het is af. Ik hou van je. Dankjewel dat ik dit heb mogen doen. 

Curriculum Vitae 
136 


\section{Curriculum Vitae}

Véronique Maria Peter Moers-Hornikx werd geboren op 5 Juli 1980 in Kigali, Rwanda als oudste dochter van Nederlandse ontwikkelingshulpmedewerkers. Ze groeide op in Rwanda en Nigeria en verhuisde in 1995 met haar ouders, zus en broers terug naar Helmond waar ze in 1998 eindexamen VWO deed aan het Carolus Borromeus College. Datzelfde jaar begon zij aan de opleiding geneeskunde aan de Universiteit Maastricht. $\mathrm{Na}$ het succesvol afleggen van het doctoraal examen in 2003 volgde zij haar coschappen in verschillende Limburgse en Brabantse ziekenhuizen. Het coschap interne geneeskunde volgde zij in het Centre Hospitalier Universitaire te Saint-Étienne, Frankrijk. In 2003 volgde zij haar wetenschapsstage onder begeleiding van prof. dr. Y. Temel, waar zij voor het eerst kennis maakte met het gebruik van diepe hersenstimulatie in diermodellen. Na afronding van het artsexamen in 2004 begon zij als arts-assistent niet in opleiding bij de vakgroep neurologie van het azM/MUMC ${ }^{+}$, waar zij in 2005 in opleiding kwam. In 2008 verrichte ze basaal onderzoek in het kader van dit proefschrift onder leiding van prof. dr. J.S.H. Vles waarvoor de opleiding neurologie tijdelijk werd onderbroken. In 2009 hervatte zij de opleiding neurologie en continueerde daarbij de wetenschappelijke activiteiten die geleid hebben tot dit proefschrift. In 2013 hoopt ze de opleiding neurologie af te ronden. In 2005 trouwde zij met Rob Moers. Zij hebben samen drie kinderen: Myrthe (5 jaar), Rianne (3 jaar) en Xander (1 jaar).

Véronique Maria Peter Moers-Hornikx was born in Kigali, Rwanda on the 5th of July 1980. She was the eldest daughter of Dutch developmental aid workers. She grew up in Rwanda and Nigeria. In 1995 she moved back to Helmond, the Netherlands, with her parents, sister and brothers. She finished her secondary school education at the Carolus Borromeus College in 1998. The same year she started her medical education at the Maastricht University. In 2003 she passed her doctoral exam, after which she followed her internships in several hospitals in the south of the Netherlands. She followed het internship internal medicine in the Centre Hospitalier Universitaire in Saint-Étienne, France. In 2003 she was first introduced to the use of deep brain stimulation in animal models by prof. dr. Y. Temel when he was her mentor during her research internship. In 2004 she finished her medical education and started as a neurology resident not in training in the azM/MUMC ${ }^{+}$, where she started her neurology training in 2005. In 2008 she performed basal research under supervision of prof. dr. J.S.H. Vles as part of this thesis for which she temporarily interrupted her neurology residency. In 2009 she again took up her residency, meanwhile continuing the research activities that have led to this thesis. She hopes to finish her neurology residency in 2013. In 2005 she married Rob Moers. They now have three children: Myrthe (5 years), Rianne (3 years) and Xander (1 year). 
